\title{
Synthesis of Z-Protected Aib- and Phe(2Me)-Containing Pentapeptides and Their Crystal Structures
}

\author{
Arnhold, Franziska S ; Linden, Anthony ; Heimgartner, Heinz
}

\begin{abstract}
A series of pentapeptide derivatives containing alpha,alpha-disubstituted alpha-amino acids have been prepared by a combination of the 'azirine/oxazolone method' and segment condensations. X-Ray crystal-structure determinations of the molecular structures confirmed the presence of helical conformations stabilized by beta-turns of type III or III'. Pentapeptides containing (R)-Phe(2Me) form a right-handed helix, whereas those containing (S)-Phe(2Me) adopt a left-handed helical structure.
\end{abstract}

DOI: https://doi.org/10.1002/hlca.201400084

Posted at the Zurich Open Repository and Archive, University of Zurich

ZORA URL: https://doi.org/10.5167/uzh-95944

Journal Article

Accepted Version

Originally published at:

Arnhold, Franziska S; Linden, Anthony; Heimgartner, Heinz (2014). Synthesis of Z-Protected Aib- and Phe(2Me)-Containing Pentapeptides and Their Crystal Structures. Helvetica Chimica Acta, 97(5):619645.

DOI: https://doi.org/10.1002/hlca.201400084 
07. 03. 2014

Prof. Dr. H. Heimgartner

Tel. 0446354282

Fax 0446356812

e-mail: heinz.heimgartner@chem.uzh.ch

Synthesis of Z-Protected Aib- and Phe(2Me)-Containing Pentapeptides and Their Crystal Structures

by Franziska S. Arnhold ${ }^{1}$ ), Anthony Linden, and Heinz Heimgartner*

Institut für Chemie der Universität Zürich, Winterthurerstrasse 190, CH-8057 Zürich,

Switzerland

${ }^{1}$ ) In part from the Ph.D. thesis of F. S. A., Universität Zürich, 1997. Present address:

Bachem AG, Hauptstrasse 144, CH-4416 Bubendorf 
A series of pentapeptide derivatives containing $\alpha, \alpha$-disubstituted $\alpha$-amino acids have been prepared by a combination of the 'azirine/oxazolone method' and segment condensations. X-ray crystal-structure determinations of the molecular structures confirmed the presence of helical conformations stabilized by $\beta$-turns of type III or III'. Pentapeptides containing $(R)$-Phe(2Me) form a right-handed helix, whereas those containing $(S)$-Phe(2Me) adopt a left-handed helical structure. 
1. Introduction. - Since the elaboration of the 'azirine/oxazolone method' as a convenient protocol for the synthesis of Aib-containing peptides [1-3], this procedure has been used successfully for the preparation of model peptides [2c][3-6], sterically congested oligopeptides [7][8], and peptaibols [2d][9-11]. It has also been shown that the method can be adopted to solid-phase methodology [12]. Another modification allowed the synthesis of endothiopeptides containing Aib units [13-16].

In the present study, a series of Z-protected pentapeptides containing $\alpha, \alpha$-disubstituted $\alpha$-amino acids were prepared according to this method. All $\alpha, \alpha$-disubstituted $\alpha$-amino acids were introduced via coupling of amino or peptide acids with 2,2-disubstituted $2 H$-azirin-3amines. The synthon for Phe(2Me) was the racemic 2-benzyl-2, $N$-dimethyl- $N$-phenyl-2Hazirin-3-amine leading to mixtures of $(R)$ - and $(S)$-Phe(2Me)-peptides $\left.{ }^{2}\right)$. In the case of diastereoisomers, they were separated chromatographically.

Based on the studies of Ramachandran et al. [18] it is well-known that the introduction of $\alpha, \alpha$-disubstituted $\alpha$-amino acids ${ }^{3)}$ into peptides stabilizes $\beta$-turn and $310^{-}$ helical conformations [19][20]. For the crystalline state, this has been proven by X-ray crystallography (e.g., [21][22]). In spite of this knowledge, current interest in conformations of small peptides is documented by several recent reports (e.g., for pentapeptides [23][24]). For this reason, we determined the crystal structures of some of the prepared pentapeptides.

2. Results and Discussion. - 2.1. Synthesis of Pentapeptides. The syntheses of the Zprotected pentapeptides were carried out via combinations of the coupling of amino or peptide acids with $2 H$-azirin-3-amines and standard coupling with amino acid esters or peptide

\footnotetext{
$\left.{ }^{2}\right)$ Synthons for enantiomerically pure $(R)$ - and $(S)$-Phe(2Me) are now also available [4a][17].

${ }^{3}$ ) The prototype is $\alpha$-aminoisobutyric acid (Aib).
} 
segments. Two examples are shown in Scheme 1: Following the procedure described in [11][25], Z-Gly-OH in THF was reacted with 2,2,N-trimethyl- $N$-phenyl-2H-azirin-3-amine (1a, Aib synthon [26]) leading to dipeptide amide 2, followed by selective hydrolysis to give Z-Gly-Aib-OH (3). Reaction of the latter with 2-benzyl-2, $N$-dimethyl- $N$-phenyl-2H-azirin-3amine (1b) [26b] yielded the tripeptide amide 4a [25a], which was hydrolyzed $(\rightarrow \mathbf{5 a})$, coupled with 1a $(\rightarrow \mathbf{6 a})$ and again hydrolyzed $(\rightarrow \mathbf{7 a})$. The coupling of 7a with H-Gly-OMe and $\mathrm{H}-\mathrm{Phe}-\mathrm{OBn}$, respectively, was carried out in $\mathrm{CH}_{2} \mathrm{Cl}_{2}$ using $\mathrm{PyBOP} / \mathrm{DIEA}^{4}$ ) as the coupling reagent, leading to pentapeptide $\mathbf{8} \mathbf{a}^{5}$ ) and $\mathbf{8 b}$, respectively. The latter was obtained as a 1:1 mixture of diastereoisomers.

Scheme 1

Similar to the preparation of $\mathbf{8 a}$ and $\mathbf{8 b}$, pentapeptide $\mathbf{8 c}$ containing 1aminocyclobutane carboxylic acid (Acb) was synthesized (Scheme 2). Coupling of 3 with 2( $N, N$-dimethylamino)-1-azaspiro[2.3]hex-1-ene (1c) [2b] gave the tripeptide amide $4 \mathbf{b}$ in $87 \%$ yield. The hydrolysis of the terminal amide group was achieved in $3 \mathrm{~N} \mathrm{HCl}\left(\mathrm{THF} / \mathrm{H}_{2} \mathrm{O}\right)$ at $40^{\circ}$ leading to $\mathbf{5 b}(96 \%)$. Subsequent azirine coupling with $\mathbf{1 a}(\rightarrow \mathbf{6 b})$, selective hydrolysis at room temperature $(\rightarrow \mathbf{7 b})$, and coupling with H-Gly-OMe by treatment with PyBOP/DIEA gave 8c.

\section{Scheme 2}

$\overline{\left.{ }^{4}\right) ~} \mathrm{PyBOP}=[($ Benzotriazol-1-yl)oxy $]$ tripyrrolidinophosphonium hexafluorophosphate; DIEA = ethyl(diisopropyl)amine (Hünig base) .

${ }^{5}$ ) The coupling of 7 with $\mathrm{H}-\mathrm{Gly}-\mathrm{OMe}$ was also achieved by treatment with $\mathrm{DCC}, \mathrm{ZnCl}_{2}$, and $\mathrm{Et}_{3} \mathrm{~N}$ in DMF [27][28] leading to $8 \mathbf{a}$ in $70 \%$ yield. 
The Pro-containing pentapeptide 8d was obtained by segment coupling of $\mathbf{3}$ and $\mathrm{H}$ Pro-Aib-Aib-N(Me)Ph (9) in $\mathrm{CH}_{2} \mathrm{Cl}_{2}$ with $\mathrm{HBPyU} / \mathrm{DIEA}^{6}$ ) as the coupling reagent [29] (Scheme 3). The tripeptide 9 [25b] was prepared by subsequent coupling of Z-Pro-OH with azirine 1a, selective hydrolysis, again coupling with 1a, and deprotection of the $N$-terminus by hydrogenolysis. Selective hydrolysis of $\mathbf{8 d}$ under the usual conditions gave the pentapeptide acid $\mathbf{1 0}$ in $\mathbf{7 0 \%}$ yield.

\section{Scheme 3}

The pentapeptides 8e and $\mathbf{8 f}$ were prepared via coupling of the dipeptide segment $\mathbf{1 1}$ [25a] with the tripeptide segment 12 and the corresponding H-Gly-Aib-Aib-N(Me)Ph [25a], respectively (Scheme 4). The coupling was performed by treatment with PyBOP/DIEA in $\mathrm{CH}_{2} \mathrm{Cl}_{2}$ at room temperature and led to $\mathbf{8 e}$ as a 1:1 mixture of diastereoisomers in almost quantitative yield. The corresponding $8 \mathbf{8 f}$ was isolated in $82 \%$ yield as a racemate. The segment 12 was obtained by coupling of $\mathbf{3}$ with H-Phe-OMe (PyBOP/DIEA; 85\%), followed by hydrogenolytic deprotection of the $\mathrm{NH}_{2}$ group (98\%). The diastereoisomers of $8 \mathbf{e}$ were separated chromatographically $\left(\mathrm{SiO}_{2}, \mathrm{AcOEt} / \mathrm{MeOH}\right)$, and the pure isomers were isolated in $47 \%((S, S)-8 \mathbf{e})$ and $44 \%((R, S)-8 \mathbf{e})$ yield. The configuration of $(S, S)-8 \mathbf{e}$ was determined by Xray crystallography (see chapter 2.3 ).

Scheme 4

$\left.{ }^{6}\right) \mathrm{HBPyU}=O-[($ Benzotriazol-1-yl)oxy $]$ dipyrrolidinocarbenium hexafluorophosphate. 
Finally, the dipeptide segment $\mathbf{1 1}$ was coupled with the tripeptides H-Pro-Aib-PheOMe (13) and the corresponding H-Pro-Aib-Aib-N(Me)Ph [25b], respectively, leading to pentapeptides $8 \mathbf{g}$ and $\mathbf{8 h}$ (Scheme 5). These couplings proved to be rather difficult. In the case of $\mathbf{8 g}$, the reaction with PyBOP/DIEA in $\mathrm{CH}_{2} \mathrm{Cl}_{2}$ for $19.5 \mathrm{~h}$ gave the product in only $39 \%$ yield, whereas with TBTU/HOBt/DIEA ${ }^{7}$ ), after 19.5 h, $8 \mathbf{g}$ was isolated in $74 \%$ yield. Also in the case of $\mathbf{8 h}$, TBTU/HOBt gave slightly better results than PyBOP/DIEA. The tripeptide $\mathbf{1 3}$ was prepared by coupling of Z-Pro-Aib-OH [25b] with H-Phe-OMe by treatment with PyBOP/DIEA.

\section{Scheme 5}

In both cases, $\mathbf{8 g}$ and $\mathbf{8 h}$, mixtures of diastereoisomers were formed, which could be separated by column chromatography $\left(\mathrm{SiO}_{2}, \mathrm{AcOEt} / \mathrm{MeOH}\right)$. The pure epimers $(R, S, S)-\mathbf{8 g}$ and $(S, S, S)-\mathbf{8 g}$ were obtained in a $\left.1.6: 1 \operatorname{ratio}^{8}\right)$. As the starting dipeptide $\mathbf{1 1}$ is a racemate, the coupling of $(R)-\mathbf{1 1}$ with $\mathbf{1 3}$ is obviously more efficient than that of $(S)$-11. A similar result was obtained in the case of $\mathbf{8 h}$ : the epimers $(R, S)-\mathbf{8 h}$ and $\left.(S, S)-\mathbf{8} \mathbf{h}^{8}\right)$ were obtained in a $2: 1$ ratio.

2.2. Conformation of the Pro-Peptide Bonds in the Pentapeptides in Solution. Whereas in general the trans-conformation of the peptide bond is highly preferred, cis- and trans-conformations of Xaa-Pro peptide bonds show comparable thermodynamic stability. This was shown first for short peptides like ( $S$-benzyl)-Cys-Pro-Leu-Gly- $\mathrm{NH}_{2}$ on the basis of

$\left.{ }^{7}\right) \quad \mathrm{TBTU}=O-(1 H$-Benzotriazol-1-yl $)-N, N, N^{\prime}, N^{\prime}$-tetramethyluronium tetrafluoroborate; $\mathrm{HOBt}=1$-hydroxybenzotriazole .

$\left.{ }^{8}\right)$ The configurations of $(R, S, S)$-8g and $(R, S)$-8h were established by X-ray crystallography (see chapter 2.3). 
their ${ }^{1} \mathrm{H}-\mathrm{NMR}$ spectra [30]. Later, it has been demonstrated that ${ }^{13} \mathrm{C}-\mathrm{NMR}$ spectroscopy is a convenient method for distinguishing between the cis- and trans-conformation of the Xaa-Pro bond [31]. Typically, the chemical shifts of $\mathrm{C}(\beta)$ (Pro) and $\mathrm{C}(\gamma)$ (Pro) in cis-Xaa-Pro peptides are $c a .31 .3$ and $22.5 \mathrm{ppm}$, respectively, whereas the corresponding values for trans-Xaa-Pro peptides are 29.5 and $24.2 \mathrm{ppm}$ [31c]. Obviously, the chemical shift difference, $\Delta \delta$, $\square \mathrm{s}$ a

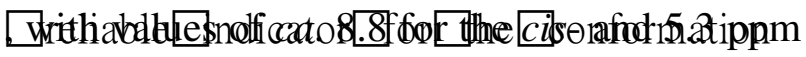
for the trans-conformation. For this reason, we analyzed the ${ }^{13} \mathrm{C}-\mathrm{NMR}$ spectra of the prepared Pro-containing pentapeptides 8d, $(R, S, S)$ - and $(S, S, S)-\mathbf{8 g},(R, S)$ - and $(S, S)-\mathbf{8 h}$, and $1 \mathbf{1 0}$ (Table 1). In all cases, only one conformation was observed with $\Delta \delta \square$ between 2.3 and 2.7 ppm, indicating the trans-conformation. This result corresponds with that of the crystal structure determination (chapter 2.3), i.e., the Xaa-Pro peptide bond adopts the same conformation in solution and in the crystal.

Table $1 .{ }^{13} C$-NMR Chemical Shifts of $C(\beta)$ and $C(\gamma)$ of the Pro Residue in ProContaining Pentapeptides

2.3. Crystal Structures of Pentapeptides. - Suitable crystals of the tetrapeptide Z-GlyAib-(R,S)-Phe(2Me)-Aib-N(Me)Ph (6a) were obtained from $\mathrm{CH}_{2} \mathrm{Cl}_{2} / \mathrm{Et}_{2} \mathrm{O} /$ hexane. The crystals are racemic and the asymmetric unit contains one peptide molecule plus one half of a disordered molecule of hexane, which sits across a centre of inversion. In Fig. 1, the peptide molecule with the $(S)$-configured amino acid Phe(2Me) is shown. The molecule forms a $\beta$ turn of type III' [33] with the Phe(2Me) unit in position (i+3) and an intramolecular H-bond

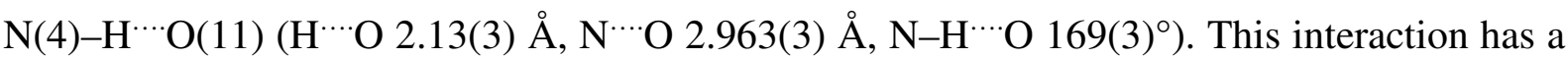
graph set motif [34] of $\mathrm{S}(10)$. A second intramolecular H-bond is formed between $\mathrm{N}(10)-\mathrm{H}$ and $\mathrm{N}(13)$. The other two NH groups are involved in intermolecular H-bonds with an adjacent 
molecule $\left(\mathrm{N}(7)-\mathrm{H}^{\cdots \cdots} \mathrm{O}\left(2^{\prime}\right), \mathrm{N}(13)-\mathrm{H}^{\cdots} \mathrm{O}\left(5^{\prime}\right)\right)$; these intermolecular interactions link the molecules into extended chains which run parallel to the [010] direction and can be described by graph set motifs of $\mathrm{C}(8)$ and $\mathrm{C}(11)$, respectively.

Fig. 1. ORTEP Plot [32] of the molecular structure of the tetrapeptide 6a (50\% probability ellipsoids, arbitrary numbering of atoms, $\mathrm{H}$-atoms bonded to $\mathrm{C}$-atoms omitted for clarity and only the major conformation of the disordered central benzyl ring is shown, solvent molecule not included)

All peptide bonds show the trans-conformation, but the Phe(2Me)-Aib bond deviates significantly from planarity $\left(\omega=155.5(2)^{\circ}\right)$. The values of the torsion angles $\omega($ Gly-Aib), $\omega\left(\right.$ Aib-Phe(2Me)), and $\omega(\mathrm{Aib}-\mathrm{N}(\mathrm{Me}) \mathrm{Ph})$ are close to $180^{\circ}(175.0(2),-176.6(2)$, and 177.5(2) $)^{\circ}$. The torsion angles $\phi$ and $\psi$ of $\operatorname{Aib}(2)\left(61.0(4)\right.$ and $\left.25.3(4)^{\circ}\right)$ and $\mathrm{Phe}(2 \mathrm{Me})$ (60.2(3) and $\left.23.2(3)^{\circ}\right)$ correspond well with the typical values of $+60^{\circ}$ and $+30^{\circ}$ for a lefthanded helical structure ( $\beta$-turn of type III') (e.g. [7][35]).

The crystals of the pentapeptide Z-Gly-Aib-( $R, S)$-Phe(2Me)-Aib-Gly-OMe (8a), obtained from $\mathrm{CH}_{2} \mathrm{Cl}_{2} /$ hexane, are also racemic. The extension of the peptide chain of $\mathbf{6 a}$ by an additional C-terminal Gly-OMe results in the formation of a $33_{10}$-helix, i.e. three consecutive $\beta$-turns of type III/III'. The molecule containing (S)-Phe(2Me) again forms a lefthanded helix with $\beta$-turns of type III' (Fig. 2, Table 2). Three intramolecular H-bonds stabilize the $33_{10}$-helix: $\mathrm{N}(1)-\mathrm{H}^{\cdots \cdots} \mathrm{O}(8), \mathrm{N}(4)-\mathrm{H}^{\cdots \cdots} \mathrm{O}(11)$, and $\mathrm{N}(7)-\mathrm{H}^{\cdots \cdots} \mathrm{O}(14)$ (Table 3). All these interactions form formal 10-membered rings (graph set motif $\mathrm{S}(10)$ ), the last one involving the $\mathrm{C}=\mathrm{O}$ group of the $\mathrm{Z}$ group. The other two $\mathrm{NH}$ groups $(\mathrm{Gly}(1)$ and $\mathrm{Aib}(2))$ are involved in intermolecular $\mathrm{H}$-bonds with the same $\mathrm{O}\left(5^{\prime}\right)$-atom of $\mathrm{Phe}(2 \mathrm{Me})$ of an adjacent molecule. This $\mathrm{O}\left(5^{\prime}\right)$-atom, therefore, accepts two H-bonds, although one of these is quite 
weak. The intermolecular H-bonds link the molecules into extended chains which run parallel to the [101] direction and can be described by graph set motifs of $C(11)$ and $C(8)$ for the interactions involving the donors Gly(1) and Aib(2), respectively.

Fig. 2. ORTEP Plot [32] of the molecular structure of the pentapeptide 8a (50\% probability ellipsoids, arbitrary numbering of atoms, $\mathrm{H}$-atoms bonded to $\mathrm{C}$-atoms omitted for clarity)

Table 2. Torsion Angles $\omega, \phi$, and $\psi$ of the Backbone of the Pentapeptides $\mathbf{6 a}, \mathbf{8 a}$, $(S, S)-\mathbf{8 e}, \mathbf{8 f},(R, S, S)-\mathbf{8 g},(R, S)-\mathbf{8 h}$, and $\mathbf{1 0}$ in the Crystal $($ Amino Acid $(i)=\mathrm{Gly}(1))$

Table 3. Intramolecular H-Bonds of Compounds 8a, $(S, S)-\mathbf{8 e}, \mathbf{8 f},(R, S, S)-\mathbf{8 g},(R, S)-\mathbf{8 h}$, and $\mathbf{1 0}$ (atom numbering refers to Figs. $1-7$ )

Racemic crystals of Z-Gly-(R,S)-Phe(2Me)-Gly-Aib-Aib-N(Me)Ph (8f) were grown from AcOEt/hexane. In Fig. 3, the molecule containing $(S)-\mathrm{Phe}(2 \mathrm{Me})$ is depicted. The asymmetric unit contains one molecule of $\mathbf{8 f}$ and approximately one third of a highly disordered hexane molecule, which sits across a centre of inversion. Four of the five NH groups act as donors for $\mathrm{H}-$ bonds. Two of them, $\mathrm{N}(1)-\mathrm{H}$ and $\mathrm{N}(4)-\mathrm{H}$, form intramolecular $\mathrm{H}-$ bonds with amide $\mathrm{O}$-atoms that are seven atoms along the peptide backbone to give a graph set motif of $\mathrm{S}(10)$ in each case (Table 3). These interactions form two $\beta$-turns of type III' (Table 2), leading to a left-handed helical structure for the molecule containing $(S)$-Phe(2Me). Surprisingly, Gly(3) does not continue this helical structure, and the $\mathrm{Z}$ group is turned away from the helix. Rather $\mathrm{N}(7)-\mathrm{H}$ of $\mathrm{Gly}(3)$ forms an intermolecular $\mathrm{H}$-bond with the terminal amide $\mathrm{O}(24)$ of a neighboring molecule. The $\mathrm{N}(13)-\mathrm{H}$ group of $\mathrm{Gly}(1)$ forms an 
intermolecular $\mathrm{H}$-bond to $\mathrm{O}(2)$ of the same neighboring molecule. These intermolecular interactions link the peptide molecules into extended chains, which run parallel to the [010] direction (graph set motifs $\mathrm{C}(11)$ and $\mathrm{C}(14)$, resp.). Interestingly, $\mathrm{N}(10)-\mathrm{H}$ is not involved in any H-bonding. Finally, $\mathrm{N}(1)-\mathrm{H}, \mathrm{N}(4)-\mathrm{H}, \mathrm{N}(7)-\mathrm{H}$, and $\mathrm{N}(10)-\mathrm{H}$ exhibit the usual weak 'sideways' contacts with one of their neighboring N-atoms. All peptide bonds are in the transconformation and differ only slightly from planarity, but the terminal amide group deviates significantly from planarity (torsion angle $\omega-153.1(8)^{\circ}$; Table 2).

Fig. 3. ORTEP Plot [32] of the molecular structure of the pentapeptide $\mathbf{8 f}$ (50\% probability ellipsoids, arbitrary numbering of atoms, $\mathrm{H}$-atoms bonded to $\mathrm{C}$-atoms omitted for clarity, solvent molecule not included)

The crystals of Z-Gly-(S)-Phe(2Me)-Gly-Aib-Phe-OMe $\quad((S, S)-8 \mathbf{e}) \quad$ are enantiomerically pure, however the absolute configuration of the molecule has not been determined. The enantiomer used in the refinement was based on the $S$-configuration at $\mathrm{C}(23)$, resulting from $(S)$-configured H-Phe-OMe used in the synthesis. Based on this, the configuration at $\mathrm{C}(9)$ of $\mathrm{Phe}(2 \mathrm{Me})$ is also $S$ (Fig. 4). The $\mathrm{Ph}$ ring of the terminal benzyl group is disordered over two orientations, with a ratio of the conformers of 0.33:0.67. The crystal lattice also contains one disordered $\mathrm{H}_{2} \mathrm{O}$ molecule per peptide molecule. All five peptide $\mathrm{NH}$ groups act as donors for H-bonding. Two of the H-bonds are intramolecular interactions with, in each case, the amide O-atom two peptide units further along the peptide chain, forming two consecutive $\beta \square$ urns of type III' (graph set motif: S(10); Table 3). As a result of the presence of $(S)$-Phe(2Me), a left-handed helical structure is formed (see e.g., [36]). The presence of $(S)$ Phe, an amino acid which usually forms right-handed helices, probably has no influence because of its position at the C-terminus of the peptide. As in $\mathbf{8 f}$, the helix is not continued by 
Gly(3), and the Z group is turned away. The NH group of Gly(3) forms an intermolecular Hbond with $\mathrm{O}\left(2^{\prime}\right)$ of $\mathrm{Aib}(4)$ of an adjacent molecule. A second intermolecular H-bond, N(13)$\mathrm{H}^{\cdots \cdots} \mathrm{O}\left(5^{\prime}\right)$, is formed with the same neighboring molecule. These two interactions link the peptide molecules into extended chains, which run parallel to the [100] direction (graph set motifs $\mathrm{C}(8)$ and $\mathrm{C}(11)$, resp.). The fifth interaction, from $\mathrm{N}(10)-\mathrm{H}$ of $\mathrm{Phe}(2 \mathrm{Me})$, involves a $\mathrm{H}_{2} \mathrm{O}$ molecule acting as an acceptor. Furthermore, analogous to 8f, N(1)-H, N(4)-H, N(7)-H, and $\mathrm{N}(10)-\mathrm{H}$ are close enough to adjacent $\mathrm{N}$-atoms in the peptide chain to form weak interactions.

Fig. 4. ORTEP Plot [32] of the molecular structure of the pentapeptide $(S, S)-\mathbf{8 e}(50 \%$ probability ellipsoids, arbitrary numbering of atoms, $\mathrm{H}$-atoms bonded to $\mathrm{C}$-atoms omitted for clarity and only the major conformation of the disordered terminal benzyl group is shown, water molecules not included)

The asymmetric unit of Z-Gly- $(R)-\mathrm{Phe}(2 \mathrm{Me})$-Pro-Aib-Phe-OMe $((R, S, S)-\mathbf{8 g})$ contains one peptide molecule, one molecule of AcOEt, and one site for a $\mathrm{H}_{2} \mathrm{O}$ molecule, which is partially occupied (50\%). The crystals are enantiomerically pure, however the absolute configuration of the molecule has not been determined. The enantiomer used in the refinement was based on the known $S$ configuration at $\mathrm{C}(6)$ and $\mathrm{C}(23)$ of the Pro and Phe residues, respectively. Based on this assumption, $\mathrm{C}(9)$ of $\mathrm{Phe}(2 \mathrm{Me})$ has the $R$ configuration. The molecule forms a right-handed $3_{10}$-helix, as expected for a peptide containing two protein amino acids and (R)-Phe(2Me) (Fig. 1, Table 2). Again, the Z-group is turned away from the helix, in this case because of the presence of Pro in position 3 of the peptide. The N(1)-H and $\mathrm{N}(4)-\mathrm{H}$ groups of Phe(5) and Aib(4), respectively, from intramolecular H-bonds with amide O-atoms that are seven atoms along the peptide backbone (graph set motif S(10) for each 
interaction, Table 3). The $\mathrm{NH}$ groups of Phe(2Me)(2) and Gly(1), N(10)-H and N(13)-H, respectively, form intermolecular H-bonds with the amide O-atoms, $\mathrm{O}\left(5^{\prime}\right)$ and $\mathrm{O}\left(2^{\prime \prime}\right)$, respectively, of different neighboring molecules. The $\mathrm{N}(10)-\mathrm{H}$ interaction links the molecules into extended chains, which run parallel to the [100] direction (graph set motif $\mathrm{C}(8)$ ), while the $\mathrm{N}(13)-\mathrm{H}$ interaction links the molecules into a second type of extended chains, which also run parallel to the [100] direction (graph set motif $\mathrm{C}(14)$ ). The $\mathrm{H}_{2} \mathrm{O}$ molecule acts as a donor for two H-bonds to amide O-atoms of two different peptide molecules (graph set motif D for each interaction). As a result, the amide $\mathrm{O}$-atoms $\mathrm{O}(2)$ of $\mathrm{Aib}(4)$ and $\mathrm{O}(11)$ of $\mathrm{Gly}(1)$ are each acceptors of two H-bonds. Neither the $\mathrm{H}_{2} \mathrm{O}$ molecule nor the AcOEt molecule acts as an acceptor for H-bonds. The combination of all intermolecular interactions links the molecules into extended sheets, which lie parallel to the (010) plane. Finally, N(1)-H and N(4)-H exhibit the usual weak 'sideways' contacts with one of their neighboring N-atoms.

Fig. 5. ORTEP Plot [32] of the molecular structure of the pentapeptide $(R, S, S)-\mathbf{8 g}$ (50\% probability ellipsoids, arbitrary numbering of atoms, $\mathrm{H}$-atoms bonded to $\mathrm{C}$-atoms omitted for clarity, solvent molecules not included)

The crystals of Z-Gly-(R)-Phe(2Me)-Pro-Aib-Aib-N(Me)Ph $(\square R \square S \square 8 h) \quad$ are enantiomerically pure, however the absolute configuration of the molecule has not been determined. The enantiomer used in the refinement was based on the known $S$ configuration of the Pro residue $(\mathrm{C}(6))$. The configuration of $\mathrm{Phe}(2 \mathrm{Me})$, at $(\mathrm{C}(9))$, is therefore $R$ (Fig. 6). The asymmetric unit contains two independent peptide molecules, one hexane molecule, and a $\mathrm{H}_{2} \mathrm{O}$ molecule, which lies on a twofold axis. The two peptide molecules have generally similar conformations with the major difference being in the orientation of the $\mathrm{Ph}$ rings at each end of the peptide chain. One of the peptide molecules (molecule B) has disorder in the 
$\mathrm{Ph}$ ring of the protecting group $\mathrm{Z}$. In molecule $\mathrm{A}$, the $\mathrm{NH}$ groups of the two Aib units, $\mathrm{N}(1)-\mathrm{H}$ and $\mathrm{N}(4)-\mathrm{H}$, form intramolecular H-bonds with $\mathrm{O}(8)$ of $\mathrm{Phe}(2 \mathrm{Me})$ and $\mathrm{O}(11)$ of Gly, respectively, resulting in two $\beta$-turns of type III (graph set motifs of $\mathrm{S}(10)$, Table 3 ). The same interactions occur in molecule $\mathrm{B}$, involving $\mathrm{N}(51)-\mathrm{H}$ and $\mathrm{N}(54)-\mathrm{H}$. These are the normal intramolecular interactions found for peptides of this type and help to form a righthanded $33_{10}$-helical structure (Table 2). These NH groups also have weak 'sideways' interactions with the next neighboring $\mathrm{N}$-atom in the chain. The other $\mathrm{NH}$ groups and the $\mathrm{H}_{2} \mathrm{O}$ molecule act as donors for intermolecular H-bonds: $\mathrm{N}(10)-\mathrm{H}$ in molecule A forms an H-bond with the amide $\mathrm{O}$-atom, $\mathrm{O}(74)$, at the opposite end of molecule $\mathrm{B}$. The corresponding group, $\mathrm{N}(60)-\mathrm{H}$, in molecule $\mathrm{B}$, however, interacts with a different $\mathrm{O}$-atom in another molecule $\mathrm{A}$, namely $\mathrm{O}(2)$. These two interactions form spiralling ${ }^{\cdots \cdots} \mathrm{A}^{\cdots \cdots} \mathrm{B}^{\cdots \cdots} \mathrm{A}^{\cdots \cdots} \mathrm{B}^{\cdots \cdots}$ chains of molecules which run parallel to the [001] direction and have a binary graph set motif of $C^{2}{ }_{2}(25)$. Furthermore, N(13)-H of molecule A forms an intermolecular H-bond with the amide O-atom of Pro(3), $\mathrm{O}(55)$, of molecule $\mathrm{B}$ and the same interaction, involving $\mathrm{N}(63)-\mathrm{H}$, occurs to link molecule $B$ to atom $\mathrm{O}(5)$ of a different molecule $A$, thus forming extended ${ }^{\cdots} A^{\cdots}{ }^{\cdots}{ }^{\cdots}{ }^{\cdots}{ }^{\cdots} B^{\cdots \cdots}$ chains of molecules, which run parallel to the [010] direction (graph set motif $\mathrm{C}^{2}, 2(22)$ ). The $\mathrm{H}_{2} \mathrm{O}$ molecule sits on a two-fold axis and therefore acts as a donor for two equivalent $\mathrm{H}$-bonds to the amide $\mathrm{O}$-atom $\mathrm{O}(52)$ of $\mathrm{Aib}(4)$ in two different B molecules (graph set motif $\mathrm{D})$. The combination of the intermolecular interactions links the molecules into three-dimensional supramolecular frameworks.

Fig. 6. ORTEP Plot [32] of the molecular structure of one of the two symmetryindependent molecules of the pentapeptide $(R, S)-\mathbf{8 h}(50 \%$ probability ellipsoids, arbitrary numbering of atoms, $\mathrm{H}$-atoms bonded to $\mathrm{C}$-atoms omitted for clarity, solvent molecules not included) 
The crystals of Z-Gly-Aib-Pro-Aib-Aib-OH (10) are enantiomerically pure, however the absolute configuration has not been determined. The enantiomer used in the refinement was based on the known $S$ configuration of Pro $(\mathrm{C}(6))$. The asymmetric unit contains one peptide and one disordered $\mathrm{MeOH}$ molecule. Two intramolecular $\mathrm{H}$-bonds, $\mathrm{N}(1)-\mathrm{H}^{\cdots} \mathrm{O}(8)$ and $\mathrm{N}(4)-\mathrm{H}^{\cdots \cdots} \mathrm{O}(11)$, form two $\beta$-turns of type III (graph set motif $\mathrm{S}(10)$, Table 3), i.e., a righthanded $310^{- \text {helix, }}$ as expected for a peptide containing $(S)$-Pro as the only chiral amino acid. The other two NH groups, $\mathrm{N}(10)-\mathrm{H}$ and $\mathrm{N}(13)-\mathrm{H}$, as well as the carboxyl OH group, $\mathrm{O}(25)-$ $\mathrm{H}$, form intermolecular $\mathrm{H}$-bonds with carbonyl O-atoms in three different neighboring molecules. The $\mathrm{O}(25)-\mathrm{H}^{\cdots \cdots} \mathrm{O}\left(5^{\prime}\right)$ interaction links the molecules into extended chains which run parallel to the [100] direction and have a graph set motif $\mathrm{C}(10))$. The $\mathrm{N}(10)-\mathrm{H}^{\cdots \cdots} \mathrm{O}(2$ ") interaction links the molecules into extended chains which run parallel to the [010] direction (graph set motif $\mathrm{C}(11)$ ), while the $\mathrm{N}(13)-\mathrm{H}^{\cdots \cdots} \mathrm{O}\left(8^{\prime \prime \prime}\right)$ interaction links the molecules into extended chains which also run parallel to the [100] direction (graph set motif $\mathrm{C}(8)$ ). The combination of these intermolecular interactions links the molecules into a two-dimensional network, which lies parallel to the (001) plane. The $\mathrm{O}(8)$-atom acts as an acceptor for two Hbonds, an intra- and an intermolecular one. Both positions for the disordered $\mathrm{MeOH}$ molecule indicate the presence of a $\mathrm{H}$-bond to the carbonyl $\mathrm{O}(14)$-atom.

Fig. 7. ORTEP Plot [32] of the molecular structure of the pentapeptide 10 (50\% probability ellipsoids, arbitrary numbering of atoms, $\mathrm{H}$-atoms bonded to $\mathrm{C}$-atoms omitted for clarity, solvent molecule not included)

3. Conclusions. - The present results prove once more the usefulness of the 'azirine/oxazolone method', combined with traditional segment coupling, in the synthesis of 
peptides containing $\alpha, \alpha$-disubstituted $\alpha$-amino acids. All of the Aib and Phe(2Me) residues, as well as the 1-aminocyclobutane carboxylic acid, have been added to $\mathrm{N}$-protected amino acids or peptides via the reaction with the corresponding 2,2-disubstituted $2 \mathrm{H}$-azirin-3-amine 1. As shown in previous studies [7][8][25][37], the coupling of two $\alpha, \alpha$-disubstituted $\alpha$ amino acids does not suffer from steric hindrance. This is of importance because coupling reactions with $N$-terminal Aib residues are known to be difficult (see e.g. [38]). As the synthon for Phe(2Me), 1b, was used as a racemate ${ }^{2}$, racemic pentapeptides such as $\mathbf{8 a}$ or mixtures of epimers such as $\mathbf{8 b}$ were formed. The pure epimers were obtained after chromatographic separation in the cases of $\mathbf{8 e}, \mathbf{8 g}$, and $\mathbf{8 h}$.

It is also important to note that the segment coupling with dipeptides with a $C$-terminal Aib or Phe(2Me) residue could be performed in good yields, even in the case of tripeptides 9 and 13 with a $\mathrm{N}$-terminal Pro. Surprisingly, the coupling with TBTU/HOBt yielded the pentapeptides $\mathbf{8 g}$ and $\mathbf{8 h}$ in significantly higher yield than with PyBOP, which was the preferred coupling reagent in the cases without Pro.

The X-ray crystal-structure determinations showed that all of the prepared pentapeptides adopt a 310 -helical conformation, as expected for Aib-containing peptides [39]. As Aib is achiral, the handedness of the helix is determined by the chiral amino acid present. It has been shown that a right-handed helix is preferred when the poly-Aib peptide contains a $N$-terminal L-amino acid or a L-amino acid within the peptide chain [40]. On the other hand, a $C$-terminal L-amino acid leads to the inverse relationship, i.e., a left-handed helix. Furthermore, it was found that the chiral $\alpha, \alpha$-disubstituted $\alpha$-amino acid Phe(2Me) has the opposite effect, i.e., an internal L-Phe(2Me) residue induces a left-handed helix [36a][41]. Recently, a similar reversal of the screw-sense induction was described for the amino acids Val and $\mathrm{Val}(2 \mathrm{Me})$ : the hexapeptides Ac-L-Xaa-(Aib) ${ }_{4}-\mathrm{Gly}_{\mathrm{NH}_{2}}$ and pentapeptides Ac-L-Xaa$(\mathrm{Aib})_{4}$-OR form left-handed helices with $\mathrm{Xaa}=$ Val but right-handed helices with $\mathrm{Xaa}=$ 
$\operatorname{Val}(2 \mathrm{Me})$ [42]. The study of the influence of the type and the position of L- and D-amino acids on the helical screw sense of Aib-containing peptides is a topic of current interest (see, e.g., [43]).

The crystal structures of the prepared pentapeptides are in full accordance with the results reported in [36][41]. Whereas the peptide $\mathbf{1 0}$ with $(S)$-Pro as the only chiral amino acid adopts a right-handed 310 -helical structure, as is 'normal' for protein amino acids, the enantiomers of the racemic peptides $8 \mathbf{a}$ and $\mathbf{8 f}$, containing $(S)$-Phe(2Me) in position 3 and 2, respectively, exist in a left-handed $3_{10}$-helical conformation in the crystal. Furthermore, in the crystal of the racemic tetrapeptide $\mathbf{6 a}$, the enantiomer with $(S)$-Phe(2Me) forms a $\beta$-turn of type III', i.e., a turn with a left-handed helical structure. On the other hand, both $(R, S, S)-\mathbf{8 g}$ and $(R, S)$-8h form right-handed $33_{10}$-helices, supported by the presence of $(R)$-Phe $(2 \mathrm{Me})$ as well as $(S)$-Pro in position 3.

It is worth mentioning that in $\mathbf{8 a}$ three intramolecular $(1 \leftarrow 4) \mathrm{H}$-bonds are formed, stabilizing the $3_{10}$ helix (Table 3). One of them is the interaction of $\mathrm{N}(7)-\mathrm{H}$ of $\mathrm{Phe}(2 \mathrm{Me})$ with $\mathrm{C}=\mathrm{O}(14)$ of the $\mathrm{Z}$ protecting group. Surprisingly, only two intramolecular $(1 \leftarrow 4) \mathrm{H}$-bonds are present in the analogous pentapeptide $\mathbf{8 f}$, and the $\mathrm{Z}$ group is not involved in an intramolecular H-bond. The analogous H-bond pattern with only two $(1 \leftarrow 4)$ interactions, is observed in the enantiomerically pure $(S, S)$-8e. In both cases, $\mathrm{N}(7)-\mathrm{H}$ of $\mathrm{Gly}(3)$ does not form intramolecular $\mathrm{H}$-bonds. In the two remaining pentapeptides $(R, S, S)-\mathbf{8 g}$ and $(R, S)-\mathbf{8 h}$, which possess a Pro(3) unit, only two intramolecular $(1 \leftarrow 4)$ H-bonds, $\mathrm{N}(1)-\mathrm{H}^{\cdots \cdots} \mathrm{O}(8)$ and $\mathrm{N}(4)-\mathrm{H}^{\cdots \cdots} \mathrm{O}(11)$, are possible.

Acknowledgement. - We thank the analytical sections of our institute for spectra and analyses, and the Stipendienfonds der Basler Chemischen Industrie and F. Hoffmann-La Roche AG, Basel, for financial support. 


\section{Experimental part}

1. Abbreviations. Acb, 1-aminocyclobutane carboxylic acid; Aib, 2-aminoisobutyric acid (2-methylalanin); CSA, camphor-10-sulfonic acid; DCC, $N, N^{\prime}$ dicyclohexylcarbodiimide; DEPC, diethyl cyanophosphonate; DIEA, ethyl(diisopropyl)amine (Hünig base); DPPA, diphenyl phosphorazidate; HBPyU, $O$-[(benzotriazol-1yl)oxy]dipyrrolidinocarbenium hexafluorophosphate; HOBt, 1-hydroxybenzotriazole; PyBOP $=[($ benzotriazol-1-yl)oxy $]$ tripyrrolidinophosphonium hexafluorophosphate; TBTU, $O-(1 H-$ benzotriazol-1-yl)- $N, N, N^{\prime}, N^{\prime}$-tetramethyluronium tetrafluoroborate; TFA, trifluoroacetic acid; Z, benzyloxycarbonyl.

2. General. See [11][25]. Amino acids were purchased from Novabiochem and Bachem and are all L-configured, other reagents and solvents from Aldrich, Bachem, Fluka and Merck, resp. Solvents were purified by standard procedures. TLC: Merck TLC glass plates, silica gel $60 F_{254}$. Preparative layer chromatography (PLC): Merck glass plates, silica gel $60 F_{254}$. Column chromatography $(\mathrm{CC})$ : Uetikon-Chemie, silica gel C-560 (0.04-0.063 mm). M.p. were measured on a Mettler-FP-5 apparatus, uncorrected. $[\alpha]_{\mathrm{D}}$-Values were determined on a Perkin-Elmer-241 polarimeter at $21^{\circ}$. IR Spectra were recorded on a PerkinElmer-781 spectrometer, in KBr. ${ }^{1} \mathrm{H}-\mathrm{NMR}$ and ${ }^{13} \mathrm{C}-\mathrm{NMR}$ spectra were recorded on a Bruker $A C-300$ or Bruker ARX-300 spectrometer at $300\left({ }^{1} \mathrm{H}\right)$ and $75.5 \mathrm{MHz}\left({ }^{13} \mathrm{C}\right)$, respectively, in $\mathrm{CDCl}_{3}, \mathrm{CD}_{3} \mathrm{OD}$ or $\left(\mathrm{D}_{6}\right)$ DMSO. The multiplicity of ${ }^{13} \mathrm{C}$ signals was determined by the DEPT technique. ESI-MS were measured on a Finnigan TSQ-700 instrument, and CI-MS (with $\mathrm{NH}_{3}$ or isobutane) on a Finnigan MAT-90 or SSQ-700 instrument; $\mathrm{m} / z$ (rel. \%).

General Procedure 1 (GP 1, Coupling with 2H-Azirin-3-amines). To a soln. of an Nprotected amino acid or $\mathrm{N}$-protected peptide $(1 \mathrm{mmol})$ in abs. THF $(5 \mathrm{ml})$ at $0^{\circ}, 1.1$ equiv. of the corresponding $2 \mathrm{H}$-azirin-3-amine in THF were added, and the mixture was stirred at r.t. 
for several $\mathrm{h}$ (Ar atmosphere). After completion of the reaction, the solvent was evaporated and the product was purified by crystallization from $\mathrm{CH}_{2} \mathrm{Cl}_{2} /$ hexane or AcOEt/hexane. In the case of incomplete reactions, the residue was dissolved in $\mathrm{CH}_{2} \mathrm{Cl}_{2}$, the remaining acid extracted with $1 \mathrm{~N}$ aq. $\mathrm{NaOH}$, the solvent evaporated, and the residue crystallized.

\section{General Procedure 2 (GP 2, Selective Hydrolysis of Peptide N-Methyl-N-} phenylamides $)$. A soln. of the peptide amide $(1 \mathrm{mmol})$ in $3 \mathrm{~N} \mathrm{HCl}\left(\mathrm{THF} / \mathrm{H}_{2} \mathrm{O}(1: 1) ; 10 \mathrm{ml}\right)$ was stirred at r.t. for 1-60 h. Then, aq. $2 \mathrm{~N} \mathrm{HCl}$ was added, and the mixture was extracted with $\mathrm{Et}_{2} \mathrm{O}$. The org. layers were combined, dried $\left(\mathrm{Na}_{2} \mathrm{SO}_{4}\right)$, and the solvent evaporated.

General Procedure 3 (GP 3, Hydrogenolytic Deprotection). The Z-protected peptide (1 mmol) was dissolved in $\mathrm{MeOH}$, and 10 weight\% $\mathrm{Pd} / \mathrm{C}(10 \%)$ was added to the soln. The mixture was stirred at r.t. under an $\mathrm{H}_{2}$ atmosphere overnight. Then, the soln. was filtered through a pad of Celite and the solvent was evaporated. The product was dried in high vacuum (i.v.).

General Procedure 4 (GP 4, Deprotection via Catalytic Hydrogen Transfer [44]). To a soln. of Z-protected peptide $(1 \mathrm{mmol})$ in $\mathrm{MeOH}$ were added 10 weight\% $\mathrm{Pd} / \mathrm{C}(10 \%)$ and $\mathrm{HCO}_{2} \mathrm{NH}_{4}(5 \mathrm{mmol})$. The mixture was heated at reflux $(10 \mathrm{~min})$ and filtered through a pad of Celite, washed with $\mathrm{MeOH}$, and the solvent was evaporated.

General Procedure 5 (GP 5, Coupling of Amino Acids). To a soln. of a Z-protected peptide $(1 \mathrm{mmol})$, an amino acid ester $(1.1 \mathrm{mmol})$, and a coupling reagent $(1 \mathrm{mmol})$ in $\mathrm{CH}_{2} \mathrm{Cl}_{2}(1 \mathrm{ml})$ was added drop-wise DIEA $(2 \mathrm{mmol} ; 3 \mathrm{mmol}$ by using the hydrochloride of the amino acid ester) at r.t. The soln. was stirred for $1 \mathrm{~h}$, and then the solvent evaporated. The residue was dissolved in AcOEt $(20 \mathrm{ml})$, washed with $5 \%$ aq. $\mathrm{KHSO}_{4}(3 \times), 5 \%$ aq. $\mathrm{NaHCO}_{3}$ $(3 \times)$, and sat. aq. $\mathrm{NaCl}$ soln., and purified by $\mathrm{CC}$.

3. Synthesis of the Pentapeptides 8. 3.1. Z-Gly-Aib-(R,S)-Phe(2Me)-Aib-Gly-OMe (8a). 3.1.1. Z-Gly-Aib-(R,S)-Phe(2Me)-N(Me)Ph (4a). According to GP 1, to a soln. of Z-Gly- 
Aib-OH (3) [25] (4.49 g, $15.3 \mathrm{mmol})$ in abs. THF (70 ml) at $0^{\circ}$ was added 2-benzyl-2,Ndimethyl- $N$-phenyl-2H-azirin-3-amine $(\mathbf{1 b} ; 15.1 \mathrm{~g}$ of a ca. 3:4 mixture of $\mathbf{1 b}$ and 2-benzyl- $N$ methyl- $N$-phenylpropanamide ( $c a .25 .9 \mathrm{mmol} \mathbf{1 b}$ )); stirring at r.t. for $70 \mathrm{~h}$. The solvent was evaporated and the product purified by $\mathrm{CC}\left(\mathrm{CH}_{2} \mathrm{Cl}_{2} / \mathrm{MeOH} 97: 3\right): 5.95 \mathrm{~g}$ (72\%) of $4 \mathbf{a}$. Colorless foam. M.p. 72.2-73.1 ${ }^{\circ}$. IR (KBr): 3320m, 3060m, 3030m, 2980m, 2940m, 1640s, $1590 s, 1515 s, 1495 s, 1450 s, 1380 m, 1365 m, 1260 m, 1240 m, 1190 m, 1155 m, 1105 m, 1080 m$, 1050m, 770m, 735m, 700s. ${ }^{1} \mathrm{H}-\mathrm{NMR}\left(\mathrm{CDCl}_{3}\right): 7.46(s, \mathrm{NH}) ; 7.4-7.2$ (m, 11 arom. H); 7.157.05 (m, 4 arom. H); 6.85 (s, NH); 5.62 (br. $s, \mathrm{NH}) ; 5.11\left(s, \mathrm{PhCH}_{2} \mathrm{O}\right) ; 3.77(d, J=5.4$, $\left.\mathrm{CH}_{2}(\mathrm{Gly})\right) ; 3.48,3.06\left(A B, J_{\mathrm{AB}}=14.2, \mathrm{PhCH}_{2}\right) ; 3.31(s, \mathrm{MeN}) ; 1.42\left(s, \mathrm{Me}_{2} \mathrm{C}\right) ; 1.33(s$, $\mathrm{Me}(\mathrm{Phe}(2 \mathrm{Me})) .{ }^{13} \mathrm{C}-\mathrm{NMR}\left(\mathrm{CDCl}_{3}\right): 172.6,172.5,168.1 \quad(3 s, 3 \mathrm{CO}$ (amide)); 156.5 ( $s$, CO(urethane)); 144.1, 136.4, 136.3 (3s, 3 arom. C); 129.8, 129.5, 128.5, 128.2, 128.1, 128.0, 127.0 (7d, 15 arom. $\mathrm{CH}) ; 67.0\left(t, \mathrm{PhCH}_{2} \mathrm{O}\right) ; 63.2,57.5$ (2s, $\left.\mathrm{C}(2)(\mathrm{Aib}), \mathrm{C}(2)(\mathrm{Phe}(2 \mathrm{Me}))\right) ; 44.9$ $\left(t, \mathrm{CH}_{2}(\mathrm{Gly})\right) ; 42.3\left(t, \mathrm{PhCH}_{2}\right) ; 41.8(q, \mathrm{MeN}) ; 24.9,24.6,23.5\left(3 q, M e_{2} \mathrm{C}, \mathrm{Me}(\mathrm{Ph}(2 \mathrm{Me}))\right) . \mathrm{CI}-$ MS: $545\left(7,[M+1]^{+}\right), 439(13), 438\left(53,[M-\mathrm{Ph}(\mathrm{Me}) \mathrm{N}]^{+}\right), 437\left(100,\left[M-\mathrm{PhCH}_{2} \mathrm{O}\right]^{+}\right), 412(8)$, 411 (15). Anal. calc. for $\mathrm{C}_{31} \mathrm{H}_{36} \mathrm{~N}_{4} \mathrm{O}_{5}$ (544.65): C 68.36, $\mathrm{H}$ 6.66, $\mathrm{N}$ 10.29; found: $\mathrm{C}$ 67.96, $\mathrm{H}$ $6.90, \mathrm{~N} 10.35$.

3.1.2. Z-Gly-Aib-(R,S)-Phe(2Me)-OH (5a). According to GP 2, a soln. of $4 \mathbf{a}(5.86 \mathrm{~g}$, $10.8 \mathrm{mmol}$ ) in $3 \mathrm{~N} \mathrm{HCl}$ was stirred at r.t. for $7 \mathrm{~h}$. The solvent was evaporated and the precipitate was filtered: $4.50 \mathrm{~g}(92 \%)$ of 5a. Colorless crystals. M.p. 192.6-193.8 ${ }^{\circ}$ IR (KBr): $3310 m, 3040 m, 2980 m, 2970 m, 1720 s, 1660 s, 1650 s, 1560 m, 1525 s, 1460 m, 1450 m, 1440 m$, 1285m, 1275m, 1245s, 1195m, 1165m, 1130m, 700m. ${ }^{1} \mathrm{H}-\mathrm{NMR}$ ((D $)$ DMSO): 12.6 (br. $s$, $\mathrm{COOH}) ; 7.96(s, \mathrm{NH}) ; 7.49(t, J=5.8, \mathrm{NH}(\mathrm{Gly})) ; 7.35-7.15(m, 8$ arom. H, NH); 7.1-7.05 (m, 2 arom. $\mathrm{H}) ; 5.00\left(s, \mathrm{PhCH}_{2} \mathrm{O}\right) ; 3.58\left(d, J=6.0, \mathrm{CH}_{2}(\mathrm{Gly})\right) ; 3.17\left(s, \mathrm{PhCH}_{2}\right) ; 1.39,1.35,1.30$ (3s, $\left.\mathrm{Me}_{2} \mathrm{C}, \mathrm{Me}(\mathrm{Phe}(2 \mathrm{Me}))\right) .{ }^{13} \mathrm{C}-\mathrm{NMR}\left(\left(\mathrm{D}_{6}\right) \mathrm{DMSO}\right): 175.2,173.5,169.0$ (3s, $2 \mathrm{CO}$ (amide), $\mathrm{COOH}) ; 156.8$ ( $s, \mathrm{CO}$ (urethane)); 137.4, 137.0 (2s, 2 arom. C); 130.7, 128.7, 128.1, 128.0, 
126.8 (5d, 10 arom. $\mathrm{CH}) ; 65.8\left(t, \mathrm{PhCH}_{2} \mathrm{O}\right) ; 59.5,56.6$ (2s, $\left.\mathrm{C}(2)(\mathrm{Aib}), \mathrm{C}(2)(\mathrm{Phe}(2 \mathrm{Me}))\right) ; 44.2$ ( $t, \mathrm{CH}_{2}(\mathrm{Gly})$ ); $41.0\left(t, \mathrm{PhCH}_{2}\right) ; 25.4,24.8,22.7$ (3q, Me $\left.2 \mathrm{C}, \mathrm{Me}(\mathrm{Phe}(2 \mathrm{Me}))\right) . \mathrm{CI}-\mathrm{MS}: 457$ (6), $456\left(24,[M+1]^{+}\right), 439(28), 438\left(100,\left[M-\mathrm{H}_{2} \mathrm{O}+1\right]^{+}\right), 348(12), 330(8), 312(12), 295$ (47), 277 (15, [M-Phe(2Me) ] ${ }^{+}, 251$ (18), 180 (30), 134 (26). Anal. calc. for $\mathrm{C}_{24} \mathrm{H}_{29} \mathrm{~N}_{3} \mathrm{O}_{6}$ (455.51): C 63.28, H 6.42, N 9.22; found: C 62.99, H 6.31, N 9.05.

3.1.3. Z-Gly-Aib-(R,S)-Phe(2Me)-Aib-N(Me)Ph (6a). According to GP 1, 5a (931 mg, $2.04 \mathrm{mmol}$ ) was reacted with 2,2,N-trimethyl- $N$-phenyl-2H-azirin-3-amine (1a [26]; $395 \mathrm{mg}$, $2.27 \mathrm{mmol})$ in THF/DMF $(20: 1 ; 21 \mathrm{ml})$ for $c a .70 \mathrm{~h}: 1.21 \mathrm{~g}(94 \%)$ of 6a. Colorless crystals. M.p. 91.3-93.0. IR (KBr): 3420m, 3320m, 3280m, 1720s, 1680s, 1650s, 1635s, 1590m, $1530 s, 1495 s, 1455 m, 1395 m, 1380 m, 1365 m, 1280 m, 1240 s, 1215 m, 1195 m, 1170 m, 1100 m$, 1090m, 1050m, 740m, 705s. ${ }^{1} \mathrm{H}-\mathrm{NMR}\left(\mathrm{CDCl}_{3}\right): 7.66(s, \mathrm{NH}) ; 7.35-7.15$ (m, 13 arom. H); 7.1-7.05 (m, 2 arom. H); 6.87, 6.56, $6.18(3 s, 3 \mathrm{NH})$; 5.03, $4.98\left(A B, J_{\mathrm{AB}}=12.2, \mathrm{PhCH}_{2} \mathrm{O}\right)$; 3.65-3.55 ( $m, \mathrm{CH}_{2}(\mathrm{Gly}), 1 \mathrm{H}$ of $\left.\mathrm{PhCH}_{2}\right) ; 3.35(s, \mathrm{MeN}) ; 3.09\left(d, J_{\mathrm{AB}}=13.7,1 \mathrm{H}\right.$ of $\left.\mathrm{PhCH}_{2}\right)$; $1.46\left(s, \mathrm{Me}_{2} \mathrm{C}\right) ; 1.42,1.38,1.27\left(3 s, \mathrm{Me}_{2} \mathrm{C}, \mathrm{Me}(\mathrm{Phe}(2 \mathrm{Me}))\right) .{ }^{13} \mathrm{C}-\mathrm{NMR}\left(\mathrm{CDCl}_{3}\right): 174.0,173.5$, 172.9, 170.3 (4s, $4 \mathrm{CO}$ (amide)); 157.1 (s, CO(urethane)); 145.6, 136.8, 136.2 (3s, 3 arom. C); $130.9,129.2,128.5,128.2,128.0,127.9,127.5,127.0,126.7(9 d, 15$ arom. CH); $67.0(t$, $\left.\mathrm{PhCH}_{2} \mathrm{O}\right) ;$ 60.2, 57.6, 57.2 (3s, $\left.2 \mathrm{C}(2)(\mathrm{Aib}), \mathrm{C}(2)(\mathrm{Phe}(2 \mathrm{Me}))\right) ; 45.3\left(t, \mathrm{CH}_{2}(\mathrm{Gly})\right) ; 40.5$ (q, $\mathrm{MeN}) ; 40.3\left(t, \mathrm{PhCH}_{2}\right) ; 25.8,25.5,24.3$ (3q, $\left.2 \mathrm{Me}_{2} \mathrm{C}, \mathrm{Me}(\mathrm{Phe}(2 \mathrm{Me}))\right)$. ESI-MS: 668 (29, $[M+\mathrm{K}]^{+}, 652\left(100,[M+\mathrm{Na}]^{+}, 523\left(47,[M-\mathrm{N}(\mathrm{Me}) \mathrm{Ph}]^{+}\right)\right.$. Anal. calc. for $\mathrm{C}_{35} \mathrm{H}_{43} \mathrm{~N}_{5} \mathrm{O}_{6}(629.76)$ : C 66.75, H 6.88, N 11.12; found: C 66.55, H 6.67, N 11.00.

Suitable crystals for the X-ray crystal-structure determination were grown from $\mathrm{CH}_{2} \mathrm{Cl}_{2} / \mathrm{Et}_{2} \mathrm{O} /$ hexane by slow evaporation of the solvent.

3.1.4. Z-Gly-Aib-(R,S)-Phe(2Me)-Aib-OH (7a). According to GP 2, 6a (4.12 g, 6.55 mmol) was hydrolyzed for 3.5 h: $3.21 \mathrm{~g}$ (91\%) of 7a. Colorless solid. M.p. 193.5-195.7 . IR (KBr): 3400m, 3300m, 2980m, 1760s, 1680s, 1650s, 1530s, 1500m, 1470m, 1455m, 1390m, 
1315m, 1280m, 1240m, 1160m, 700m. ${ }^{1} \mathrm{H}-\mathrm{NMR}\left(\left(\mathrm{D}_{6}\right) \mathrm{DMSO}\right): 8.27(s, \mathrm{NH}) ; 7.51(t, J=5.5$, $\mathrm{NH}(\mathrm{Gly})) ; 7.47$ (s, NH); 7.4-7.2 (m, 8 arom. H); 7.15-7.1 (m, 2 arom. H); $6.97(s, \mathrm{NH}) ;$ 5.01, $4.98\left(A B, J_{\mathrm{AB}}=13.4, \mathrm{PhCH}_{2} \mathrm{O}\right) ; 3.7-3.5\left(A B X, J_{\mathrm{AB}}=16.3, \mathrm{CH}_{2}(\mathrm{Gly})\right) ; 3.40,2.99\left(A B, J_{\mathrm{AB}}=\right.$ 13.4, $\left.\mathrm{PhCH}_{2}\right) ; 1.36,1.35,1.33,1.30,1.22\left(5 s, 2 \mathrm{Me}_{2} \mathrm{C}, \mathrm{Me}(\mathrm{Phe}(2 \mathrm{Me}))\right) .{ }^{13} \mathrm{C}-\mathrm{NMR}\left(\mathrm{CDCl}_{3}\right)$ : 175.5, 172.82, 172.78, 169.9 (4s, $3 \mathrm{CO}$ (amide), $\mathrm{COOH}$ ); 156.6 ( $s, \mathrm{CO}$ (urethane)); 137.0, 136.7 (2s, 2 arom. C); 130.7, 128.2, 127.73, 127.68, 127.5, 126.1 (6d, 10 arom. CH); 65.6 (t, $\left.\mathrm{PhCH}_{2} \mathrm{O}\right)$; 58.7, 56.1, 54.7 (3s, $\left.2 \mathrm{C}(2)(\mathrm{Aib}), \mathrm{C}(2)(\mathrm{Phe}(2 \mathrm{Me}))\right) ; 43.6\left(t, \mathrm{CH}_{2}(\mathrm{Gly})\right) ; 39.2(t$, $\left.\mathrm{PhCH}_{2}\right)$; 25.6, 24.9, 24.3, 24.2, 23.2 (5q, $2 \mathrm{Me}_{2} \mathrm{C}$, Me(Phe(2Me))). ESI-MS: 563 (100, $\left.[M+\mathrm{Na}]^{+}\right)$. Anal. calc. for $\mathrm{C}_{28} \mathrm{H}_{36} \mathrm{~N}_{4} \mathrm{O}_{7}$ (540.62): C 62.21, H 6.71, N 10.36; found: C 62.15, H 6.70, N 10.34 .

3.1.5. Z-Gly-Aib-(R,S)-Phe(2Me)-Aib-Gly-OMe (8a). According to GP 5, 7a (2.46 g, $4.54 \mathrm{mmol})$ was coupled with H-Gly-OMe.HCl $(639 \mathrm{mg}, 5.09 \mathrm{mmol})$ by treatment with PyBOP (2.22 g, $4.63 \mathrm{mmol})$ and DIEA (1.81 g, $13.97 \mathrm{mmol})$ in $\mathrm{CH}_{2} \mathrm{Cl}_{2}(7 \mathrm{ml})$; $1 \mathrm{~h}$. CC with AcOEt gave 2.54 g (91\%) 8a. Colorless foam. M.p. 160.3-161.3․ IR (KBr): 3340m, 3320s, $3000 s, 2960 m, 1755 s, 1705 s, 1675 s, 1650 s, 1530 s, 1460 m, 1385 m, 1365 m, 1305 m, 1290 m$, 1260m, 1240m, 1215m, 1195m, 1180m, 1160m, 975m, 740m, 710m, 700m. ${ }^{1} \mathrm{H}-\mathrm{NMR}\left(\mathrm{CDCl}_{3}\right)$ : $7.81(t, \mathrm{NH}(\mathrm{Gly})) ; 7.41(s, \mathrm{NH}) ; 7.35-7.2$ ( $m, 8$ arom. H); 7.1-7.05 (m, 2 arom. H); 6.90, 6.82 $(2 s, 2 \mathrm{NH}) ; 6.08$ (t-like, $\mathrm{NH}(\mathrm{Gly})) ; 4.97\left(s, \mathrm{PhCH}_{2} \mathrm{O}\right) ; 3.95\left(d, J=5.9, \mathrm{CH}_{2}(\mathrm{Gly})\right) ; 3.71-3.70$ $\left(m, \mathrm{CH}_{2}(\mathrm{Gly})\right) ; 3.56(s, \mathrm{MeO}) ; 3.23,3.03\left(A B, J_{\mathrm{AB}}=13.7, \mathrm{PhCH}_{2}\right) ; 1.49,1.46,1.39,1.37(4 s$, $\left.2 \mathrm{Me}_{2} \mathrm{C}, \mathrm{Me}(\mathrm{Phe}(2 \mathrm{Me}))\right) .{ }^{13} \mathrm{C}-\mathrm{NMR}\left(\mathrm{CDCl}_{3}\right): 176.2,174.8,173.8,171.5,170.5$ (5s, 4 CO(amide), COOMe); 157.3 ( $s, \mathrm{CO}($ urethane)); 136.1, 136.0 (2s, 2 arom. C); 130.8, 128.6, 128.3, 128.1, 127.9, 127.0 (6d, 10 arom. $\mathrm{CH}) ; 67.1\left(t, \mathrm{PhCH}_{2} \mathrm{O}\right) ; 59.9,57.3,57.0(3 s, 2$ $\mathrm{C}(2)(\mathrm{Aib}), \mathrm{C}(2)(\mathrm{Phe}(2 \mathrm{Me}))) ; 52.2$ ( $s, \mathrm{MeO}) ; 45.4,41.4,41.1$ (3t, $\left.2 \mathrm{CH}_{2}(\mathrm{Gly}), \mathrm{PhCH}_{2}\right) ; 26.2$, 25.4, 24.5, $23.4\left(4 q, 2 \mathrm{Me}_{2} \mathrm{C}, \mathrm{Me}(\mathrm{Phe}(2 \mathrm{Me}))\right)$. ESI-MS: $650\left(5,[M+\mathrm{K}]^{+}\right), 634(100$, 
$\left.[M+\mathrm{Na}]^{+}\right], 612\left(3,[M+1]^{+}\right)$. Anal. calc. for $\mathrm{C}_{31} \mathrm{H}_{41} \mathrm{~N}_{5} \mathrm{O}_{8}$ (611.69): C 60.87, H 6.76, N 11.45; found: C 60.78, H 6.63, N 11.44.

Suitable crystals for the X-ray crystal-structure determination were grown from $\mathrm{CH}_{2} \mathrm{Cl}_{2}$ /hexane by slow evaporation of the solvent.

3.2. Z-Gly-Aib-(R,S)-Phe(2Me)-Aib-Phe-OBn (8b). According to GP 5, 7a (432 mg, $0.779 \mathrm{mmol})$ was coupled with $\mathrm{H}-\mathrm{Phe}-\mathrm{OBn} . \mathrm{HCl}(250 \mathrm{mg}, 0.857 \mathrm{mmol})$ by treatment with PyBOP (373 mg, $0.779 \mathrm{mmol})$ and DIEA (307 mg, $2.205 \mathrm{mmol})$ in $\mathrm{CH}_{2} \mathrm{Cl}_{2}(3 \mathrm{ml}) ; 5.5 \mathrm{~h}$. CC with AcOEt/hexane 3:1 gave $527 \mathrm{mg}(85 \%) \mathbf{8 b}$ (mixture of diastereoisomers). Colorless solid. M.p. $134.0-136.5^{\circ} \cdot[\alpha]_{\mathrm{D}}=-20.3(\mathrm{c}=0.5, \mathrm{EtOH}) . \mathrm{IR}(\mathrm{KBr}): 3300 m, 1740 m, 1700 m, 1660 s$, 1535s, 1500m, 1455m, 1275m, 1240m, 700m. ${ }^{1} \mathrm{H}-\mathrm{NMR}\left(\mathrm{CDCl}_{3}\right): 7.78(m, \mathrm{NH}) ; 7.46,7.36$ $(2 s, 1 \mathrm{NH}) ; 7.25-7.15$ (m, 18 arom. H); 7.1-7.05 (m, 2 arom. H); 6.69, 6.93 (2s, $1 \mathrm{NH}) ; 6.75$, $6.71(2 s, 1 \mathrm{NH}) ; 6.22,6.17(2$ br. $s, 1 \mathrm{NH}) ; 5.01\left(s, \mathrm{PhCH}_{2} \mathrm{O}\right) ; 5.0-4.9\left(m, \mathrm{PhCH}_{2} \mathrm{O}\right) ; 4.75-$ $4.65(m, \mathrm{CH}(\mathrm{Phe})) ; 3.65-3.6\left(m, \mathrm{CH}_{2}(\mathrm{Gly})\right) ; 3.37,3.2-3.1,3.02(t, m, d, J=15.6,13.7,1: 2: 1,2$ $\left.\mathrm{PhCH}_{2}\right) ; 1.46,1.44,1.40,1.37,1.34,1.33\left(6 s, 2 \mathrm{Me}_{2} \mathrm{C} ; \mathrm{Me}(\mathrm{Phe}(2 \mathrm{Me}))\right) .{ }^{13} \mathrm{C}-\mathrm{NMR}\left(\mathrm{CDCl}_{3}\right)$ : 175.7, 174.1, 173.0, 172.1, 170.3 (5s, $4 \mathrm{CO}$ (amide), COOBn); 157.3 ( $s, \mathrm{CO}$ (urethane)); 137.1, 136.1, 135.5 (3s, 3 arom. C); 130.9, 129.3, 129.2, 128.5, 128.4, 128.3, 128.12, 128.09, 128.0, 126.9, 126.6 (11d, 20 arom. $\mathrm{CH}) ; 67.1,66.8,66.7$ (3t, $\left.2 \mathrm{PhCH}_{2} \mathrm{O}\right) ; 59.9,57.0$ (2s, 2 $\mathrm{C}(2)(\mathrm{Aib}), \mathrm{C}(2)(\mathrm{Phe}(2 \mathrm{Me}))) ; 54.5$ (d, $\mathrm{CH}(2) \mathrm{Phe}) ; 4$ 45.4, 37.5 (2t, $\left.\mathrm{CH}_{2}(\mathrm{Gly}), 2 \mathrm{PhCH}_{2}\right) ; 25.7$, 25.0, 24.5, 24.1, $23.4\left(5 q, 2 \mathrm{Me}_{2} \mathrm{C}, \mathrm{Me}(\mathrm{Phe}(2 \mathrm{Me}))\right)$. ESI-MS: $801\left(100,[\mathrm{M}+\mathrm{Na}]^{+}\right)$. Anal. calc. for $\mathrm{C}_{44} \mathrm{H}_{51} \mathrm{~N}_{5} \mathrm{O}_{8}$ (777.92): C 67.94, H 6.61, N 9.00; found: C 67.79, H 6.88, N 9.00.

3.3. Z-Gly-Aib-Acb-Aib-Gly-OMe (8c). 3.3.1. Z-Gly-Aib-Acb-NMe 2 (4b). According to GP 1, 3 (1.68 g, $5.72 \mathrm{mmol})$ was reacted with 2-dimethylamino-1-azabicyclo[2.3]hex-1-ene (1c) [2b] (782 mg, $6.30 \mathrm{mmol})$ in THF $(16 \mathrm{ml})$; $c a .1 \mathrm{~h}$. The precipitate was filtered and washed with $\mathrm{Et}_{2} \mathrm{O}: 1.02 \mathrm{~g}$ of 8c. $\mathrm{CC}\left(\mathrm{CH}_{2} \mathrm{Cl}_{2} / \mathrm{MeOH} 10: 1\right)$ of the filtrate gave another $1.06 \mathrm{~g}$ of 8c. Total yield: 2.08 g (87\%). Colorless solid. M.p. 212.3-213.7. IR (KBr): 3340m, 3270s, 
$3050 m, 2980 m, 2940 m, 1730 s, 1710 m, 1680 m, 1660 s, 1630 s, 1540 m, 1530 m, 1395 m, 1270 m$, 1245m, 1230m, 750w, 700w. ${ }^{1} \mathrm{H}-\mathrm{NMR}\left(\left(\mathrm{D}_{6}\right) \mathrm{DMSO}\right): 7.90,7.89$ (2s, $\left.2 \mathrm{NH}\right) ; 7.56$ ( $t$-like, NH); 7.35-7.3 (m, 5 arom. H); $5.05\left(s, \mathrm{PhCH}_{2} \mathrm{O}\right) ; 3.61\left(d, J=5.8, \mathrm{CH}_{2}(\mathrm{Gly})\right) ; 3.33\left(s, \mathrm{Me}_{2} \mathrm{~N}\right) ; 2.6-$ 2.5, 2.2-2.1, 1.76-1.74, 1.6-1.55 (4m, 2:2:1:1, $\left.3 \mathrm{CH}_{2}(\mathrm{Acb})\right) ; 1.36\left(s, \mathrm{Me}_{2} \mathrm{C}\right) .{ }^{13} \mathrm{C}-\mathrm{NMR}$ ((D) DMSO): 175.2, 170.6, 168.4 (3s, $3 \mathrm{CO}$ (amide)); 156.6 (s, CO(urethane)); 136.9 (s, 1 arom. C); 128.3, 127.7, 127.5 (3d, 5 arom. $\mathrm{CH}) ; 65.4\left(t, \mathrm{PhCH}_{2} \mathrm{O}\right) ; 58.2,55.7$ (2s, $\mathrm{C}(2)$ (Aib), $\mathrm{C}(2)(\mathrm{Acb})) ; 44.0\left(t, \mathrm{CH}_{2}(\mathrm{Gly})\right) ; 36.8,36.0\left(2 q, \mathrm{Me}_{2} \mathrm{~N}\right) ; 30.9\left(t, 2 \mathrm{CH}_{2}(\mathrm{Acb})\right) ; 24.8\left(q, M e_{2} \mathrm{C}\right)$, $14.3\left(t, \mathrm{CH}_{2}(\mathrm{Acb})\right)$. ESI-MS: $441\left(100,[M+\mathrm{Na}]^{+}\right)$.

3.3.2. Z-Gly-Aib-Acb-OH (5b). According to $G P$ 2, $4 \mathbf{b}(372 \mathrm{mg}, 0.890 \mathrm{mmol})$ was hydrolyzed for $70 \mathrm{~h}$ at $40^{\circ}: 334 \mathrm{mg}(96 \%)$ of 5b. Colorless crystals. M.p. 180.5-182.3 . IR (KBr): 3340s, 3290s, 3060m, 2980m, 2950m, 1720s, 1680s, 1670s, 1650s, 1565m, 1550s, $1540 s, 1525 m, 1470 m, 1430 s, 1385 m, 1280 s, 1250 s, 1235 m, 1165 m, 1110 m, 750 m .{ }^{1} \mathrm{H}-\mathrm{NMR}$ (CD $3 \mathrm{OD}):$ 7.4-7.25 (m, 5 arom. $\mathrm{H}) ; 5.11\left(s, \mathrm{PhCH}_{2} \mathrm{O}\right) ; 3.72\left(s, \mathrm{CH}_{2}(\mathrm{Gly})\right)$; 2.65-2.5, 2.4-2.3, 2.05-1.85 (3m, $\left.3 \mathrm{CH}_{2}(\mathrm{Acb})\right) ; 1.44$ (s, $\left.\mathrm{Me}_{2} \mathrm{C}\right) .{ }^{13} \mathrm{C}-\mathrm{NMR}\left(\mathrm{CD}_{3} \mathrm{OD}\right): 175.5,174.8,170.1(3 s, 2$ $\mathrm{CO}$ (amide), $\mathrm{COOH}) ; 157.8$ ( $s, \mathrm{CO}$ (urethane)); 136.6 ( $s, 1$ arom. C); 128.0, 127.5, 127.3 (3d, 5 arom. $\mathrm{CH}) ; 66.3\left(t, \mathrm{PhCH}_{2} \mathrm{O}\right) ; 58.2,56.2$ (2s, C(2)(Aib), $\left.\mathrm{C}(2)(\mathrm{Acb})\right) ; 44.0\left(t, \mathrm{CH}_{2}(\mathrm{Gly})\right) ; 30.5$ $\left(t, 2 \mathrm{CH}_{2}(\mathrm{Acb})\right) ; 23.8\left(q, \mathrm{Me}_{2} \mathrm{C}\right), 14.8\left(t, \mathrm{CH}_{2}(\mathrm{Acb})\right)$. ESI-MS: $430\left(51,[M+\mathrm{K}]^{+}\right), 414(100$, $\left.[M+\mathrm{Na}]^{+}\right)$. Anal. calc. for $\mathrm{C}_{19} \mathrm{H}_{25} \mathrm{~N}_{3} \mathrm{O}_{6} \cdot 0.5 \mathrm{H}_{2} \mathrm{O}$ (400.43): C 56.99, H 6.54, N 10.49; found: C 57.25, H 6.77, N 10.76.

3.3.3. Z-Gly-Aib-Acb-Aib-N(Me)Ph (6b). According to GP 1, 5b (840 mg, $2.15 \mathrm{mmol})$ was reacted with 1a $(411 \mathrm{mg}, 2.36 \mathrm{mmol})$ in THF/DMF (29 ml, 28:1). CC $\left(\mathrm{CH}_{2} \mathrm{Cl}_{2} / \mathrm{MeOH}\right.$ 10:1) gave 1.14 g (94\%) of 6b. Colorless solid. Phase transitions at 137 and 167 ${ }^{\circ}$; m.p. $187.8-$ 188.3․ IR (KBr): 3300s, 3060m, 2990m, 2940m, 1710s, 1680s, 1675s, 1670s, 1660s, 1650s, $1645 s, 1630 s, 1605 m, 1590 m, 1550 s, 1540 s, 1530 s, 1520 s, 1505 s, 1495 s, 1470 s, 1395 m$, $1275 m, 1265 m, 1240 s, 1190 m, 1155 m, 1090 m, 700 m .{ }^{1} \mathrm{H}-\mathrm{NMR}\left(\mathrm{CD}_{3} \mathrm{OD}\right): 7.51$ (br. $\left.s, 2 \mathrm{NH}\right)$; 
7.4-7.2 (m, 10 arom. H); 6.58 (br. $s, \mathrm{NH}) ; 6.44(s, \mathrm{NH}) ; 5.15\left(s, \mathrm{PhCH}_{2} \mathrm{O}\right) ; 3.70(d, J=5.2$, $\mathrm{CH}_{2}$ (Gly)); 3.30 (s, MeN); 2.7-2.55, 2.15-1.95, 1.95-1.8 (3m, 2:3:1, $3 \mathrm{CH}_{2}$ (Acb)); 1.44, 1.28 $\left(2 s, 2 \mathrm{Me}_{2} \mathrm{C}\right) .{ }^{13} \mathrm{C}-\mathrm{NMR}\left(\mathrm{CDCl}_{3}\right): 174.0,173.7,173.5,170.7$ (4s, $4 \mathrm{CO}$ (amide)); 157.6 ( $s$, $\mathrm{CO}($ urethane)); 146.0, 136.5 (2s, 2 arom. C); 129.2, 128.5, 128.2, 127.8, 127.3, 127.0 (6d, 10 arom. $\mathrm{CH}) ; 67.0\left(t, \mathrm{PhCH}_{2} \mathrm{O}\right) ; 59.2,56.8,56.6$ (3s, $\left.2 \mathrm{C}(2)(\mathrm{Aib}), \mathrm{C}(2)(\mathrm{Acb})\right) ; 45.7$ ( $t$, $\left.\mathrm{CH}_{2}(\mathrm{Gly})\right) ; 40.5$ (q, MeN); 31.6, 31.3 (2t, $\left.2 \mathrm{CH}_{2}(\mathrm{Acb})\right) ; 25.7,24.9$ (2q, $\left.2 \mathrm{Me}_{2} \mathrm{C}\right), 16.0$ ( $t$, $\left.\mathrm{CH}_{2}(\mathrm{Acb})\right)$. ESI-MS: $588\left(100,[M+\mathrm{Na}]^{+}\right), 459\left(5,[M-\mathrm{N}(\mathrm{Me}) \mathrm{Ph}]^{+}\right), 374(26,[M-\mathrm{Aib}-$ $\left.\left.\mathrm{N}(\mathrm{Me}) \mathrm{Ph}]^{+}\right), 277(3,[M-\mathrm{Acb}-\mathrm{Aib}-\mathrm{N}(\mathrm{Me}) \mathrm{Ph})]^{+}\right)$.

3.3.4. Z-Gly-Aib-Acb-Aib-OH (7b). According to GP 2, $6 \mathbf{b}(1.033 \mathrm{~g}, 1.83 \mathrm{mmol})$ was hydrolyzed and the product extracted with AcOEt: $828 \mathrm{mg}(95 \%)$ of 7b. Colorless solid. M.p. 201.4-203.6. IR (KBr): 3350m, 3320s, 3270s, 3000m, 2960m, 1730s, 1705s, 1670s, 1660s, $1650 s, 1635 s, 1605 m, 1545 s, 1540 s, 1530 s, 1470 m, 1465 m, 1395 m, 1330 m, 1310 m, 1290 m$, $1260 s, 1245 s, 1215 m, 1170 m, 1150 m, 970 m, 745 m .{ }^{1} \mathrm{H}-\mathrm{NMR}\left(\mathrm{CD}_{3} \mathrm{OD}\right): 8.40(s, 0.3 \mathrm{NH})$; $7.98(s, \mathrm{NH}) ; 7.34(s, 0.7 \mathrm{NH}) ; 7.4-7.25\left(m, 10\right.$ arom. H); $5.08\left(s, \mathrm{PhCH}_{2} \mathrm{O}\right) ; 3.71(s$, $\mathrm{CH}_{2}$ (Gly)); 2.7-2.6, 2.25-2.1, 2.0-1.9 (3m, $2 \mathrm{H}$ each, $3 \mathrm{CH}_{2}(\mathrm{Acb}) ; 1.47,1.44\left(2 s, 2 \mathrm{Me}_{2} \mathrm{C}\right)$. ${ }^{13} \mathrm{C}-\mathrm{NMR}\left(\mathrm{CD}_{3} \mathrm{OD}\right): 178.2,176.3,175.3,172.5$ (4s, $3 \mathrm{CO}$ (amide), $\left.\mathrm{COOH}\right) ; 159.5$ ( $s$, $\mathrm{CO}($ urethane)); 138.1 ( $s, 1$ arom. C); 129.5, $129.1,128.9$ (3d, 5 arom. $\mathrm{CH}) ; 68.1\left(t, \mathrm{PhCH}_{2} \mathrm{O}\right)$; 60.5, 57.7, 57.1 (3s, $2 \mathrm{C}(2)(\mathrm{Aib}), \mathrm{C}(2)(\mathrm{Acb})) ; 45.3\left(t, \mathrm{CH}_{2}(\mathrm{Gly})\right) ; 31.9\left(t, 2 \mathrm{CH}_{2}(\mathrm{Acb})\right) ; 25.4$ $\left(q, 2 \mathrm{Me}_{2} \mathrm{C}\right) ; 16.5\left(t, \mathrm{CH}_{2}(\mathrm{Acb})\right)$. ESI-MS: $499\left(100,[M+\mathrm{Na}]^{+}\right)$. Anal. calc. for $\mathrm{C}_{23} \mathrm{H}_{32} \mathrm{~N}_{4} \mathrm{O}_{7}$ (476.53): C 57.97, H 6.77, N 11.76; found: C 57.76, H 6.84, N 11.31.

3.3.5. Z-Gly-Aib-Acb-Aib-Gly-OMe (8c). According to GP 5, 7b (268 mg, 0.653 mmol) and H-Gly-OMe.HCl (79 mg, $0.629 \mathrm{mmol})$ were treated with HBPyU (243 mg, 0.564 mmol) and DIEA (220 mg, $1.702 \mathrm{mmol})$ for $0.5 \mathrm{~h}$. CC (AcOEt/MeOH 20:1) gave $255 \mathrm{mg}$ (83\%) of 8c. Colorless solid. M.p. 160.0-161.4 ${ }^{\circ}$ IR (KBr): 3340s, 3280s, 3060m, 2980m, 2950m, 1740s, 1710s, 1685s, 1650s, 1630s, 1550s, 1540s, 1530s, 1505m, 1470m, 1465m, 
$1455 m, 1440 m, 1380 m, 1365 m, 1310 m, 1275 s, 1240 s, 1220 m, 1195 m, 1170 m, 1155 m, 1050 m$, 700m. ${ }^{1} \mathrm{H}-\mathrm{NMR}\left(\mathrm{CDCl}_{3}\right): 7.52(s, \mathrm{NH}) ; 7.46(t, J=5.8, \mathrm{NH}(\mathrm{Gly})) ; 7.4-7.35$ ( $m, 5$ arom. H, 1 $\mathrm{NH}) ; 6.90(s, \mathrm{NH}) ; 6.31(t$-like, $\mathrm{NH}) ; 5.14\left(s, \mathrm{PhCH}_{2} \mathrm{O}\right) ; 3.98\left(d, J=5.8, \mathrm{CH}_{2}(\mathrm{Gly})\right) ; 3.75(d, J$ $\left.=5.2, \mathrm{CH}_{2}(\mathrm{Gly})\right) ; 3.65$ ( $\left.s, \mathrm{MeO}\right) ; 2.75-2.7,2.05-1.85$ (2m, 1:2, $3 \mathrm{CH}_{2}(\mathrm{Acb}) ; 1.55,1.46$ (2s, 2 $\left.\mathrm{Me}_{2} \mathrm{C}\right) .{ }^{13} \mathrm{C}-\mathrm{NMR}\left(\mathrm{CDCl}_{3}\right): 176.5,175.3,173.6,171.0,170.6$ (5s, $4 \mathrm{CO}$ (amide), COOMe); $157.8(s, \mathrm{CO}$ (urethane)); $136.4(s, 1$ arom. C); 128.6, 128.2, 127.6 (3d, 5 arom. $\mathrm{CH}) ; 67.2(t$, $\left.\mathrm{PhCH}_{2} \mathrm{O}\right) ; 59.2,57.0,56.6(3 s, 2 \mathrm{C}(2)(\mathrm{Aib}), \mathrm{C}(2)(\mathrm{Acb})) ; 52.1(q, \mathrm{MeO}) ; 45.8,41.4(2 t, 2$ $\left.\mathrm{CH}_{2}(\mathrm{Gly})\right) ; 30.9$ ( $\left.t, 2 \mathrm{CH}_{2}(\mathrm{Acb})\right) ; 25.3,24.7$ (2q, $\left.2 \mathrm{Me}_{2} \mathrm{C}\right) ; 15.4\left(t, \mathrm{CH}_{2}(\mathrm{Acb})\right)$. ESI-MS: 570 $\left(100,[M+\mathrm{Na}]^{+}\right)$.

3.4. Z-Gly-Aib-Pro-Aib-Aib-N(Me)Ph (8d). According to GP 5, 3 (1.18 g, $4.00 \mathrm{mmol})$ and H-Pro-Aib-Aib-N(Me)Ph [25b] (1.65 g, $4.40 \mathrm{mmol})$ in $\mathrm{CH}_{2} \mathrm{Cl}_{2}(20 \mathrm{ml})$ were treated with HBPyU (1.72 g, $4.00 \mathrm{mmol})$ and DIEA $(1.03 \mathrm{~g}, 8.00 \mathrm{mmol})$ for $15 \mathrm{~h}$. CC $\left(\mathrm{CH}_{2} \mathrm{Cl}_{2} / \mathrm{MeOH}\right.$ 20:1) gave $2.15 \mathrm{~g}(82 \%)$ of 8d. Colorless solid. M.p. 107.6-108.7 $.[\alpha]_{\mathrm{D}}=+34.5(\mathrm{c}=1.04$, EtOH). IR (KBr): 3420m, 3300s, 3060m, 2980m, 2930m, 1725s, 1660s, 1625s, 1595m, 1540s, $1520 s, 1495 s, 1470 m, 1455 m, 1410 m, 1395 m, 1365 m, 1280 m, 1240 s, 1215 m, 1170 m, 1090 m$, 1050m, 705m, 700m. ${ }^{1} \mathrm{H}-\mathrm{NMR}\left(\mathrm{CDCl}_{3}\right): 7.57(s, \mathrm{NH}) ; 7.41(s, \mathrm{NH}) ; 7.4-7.15(m, 10$ arom. H, $1 \mathrm{NH}) ; 6.14$ (br. $s, \mathrm{NH}) ; 5.10,5.08\left(A B, J_{\mathrm{AB}}=12.0, \mathrm{PhCH}_{2} \mathrm{O}\right) ; 4.2-4.15\left(m, \mathrm{CH}_{2}(2)\right.$ (Pro)); 3.8-3.55 ( $m, \mathrm{CH}_{2}(\mathrm{Gly}), 1 \mathrm{H}$ of $\left.\mathrm{CH}_{2}(5)(\mathrm{Pro})\right) ; 3.38(s, \mathrm{MeN}) ; 3.35-3.2$ ( $m, 1 \mathrm{H}$ of $\mathrm{CH}_{2}(5)$ (Pro)); 2.35-2.2, 1.95-1.8, 1.75-1.65 (3m, $\mathrm{CH}_{2}(3), \mathrm{CH}_{2}(4)$ (Pro)); 1.60, 1.58, 1.49, $1.43,1.28\left(5 s, 1: 1: 1: 2: 1,3 \mathrm{Me}_{2} \mathrm{C}\right) .{ }^{13} \mathrm{C}-\mathrm{NMR}\left(\mathrm{CDCl}_{3}\right): 175.1,174.3,173.3,171.7,169.7(5 s, 5$ $\mathrm{CO}$ (amide)); 156.9 (s, $\mathrm{CO}($ urethane)); 145.5, 136.5 (2s, 2 arom. C); 129.1, 128.5, 128.2, 128.0, 127.2, 126.6 (6d, 10 arom. $\mathrm{CH}) ; 66.9\left(t, \mathrm{PhCH}_{2} \mathrm{O}\right) ; 64.2(d, \mathrm{CH}(2)(\mathrm{Pro})) ;$ 57.1, 57.0, 56.5 (3s, $3 \mathrm{C}(2)(\mathrm{Aib})) ; 48.7\left(t, \mathrm{CH}_{2}(5)(\mathrm{Pro})\right) ; 44.1$ ( $\left.t, \mathrm{CH}_{2}(\mathrm{Gly})\right) ; 40.1$ ( $\left.s, \mathrm{MeN}\right) ; 28.8,26.2$ (2t, $\left.\mathrm{CH}_{2}(3), \mathrm{CH}_{2}(4)(\mathrm{Pro})\right) ; 27.8,26.1,25.8,25.6,23.9,23.6$ (6q, $\left.3 \mathrm{Me}_{2} \mathrm{C}\right)$. ESI-MS: 673 (100, $\left.[M+\mathrm{Na}]^{+}\right)$ 
3.5. Z-Gly-Aib-Pro-Aib-Aib-OH (10). According to GP 2, 8d (1.03 g, $1.58 \mathrm{mmol})$ was hydrolyzed for $4.5 \mathrm{~h}$. The precipitate was filtered (549 $\mathrm{mg}$ of 10) and the filtrate purified by $\mathrm{CC}\left(\mathrm{CH}_{2} \mathrm{Cl}_{2} / \mathrm{MeOH}\right.$ 10:1). Total yield of 10: $629 \mathrm{mg}$ (70\%). Colorless solid. M.p. 192.3193.6 $.[\alpha]_{\mathrm{D}}=+22.4(\mathrm{c}=1.02, \mathrm{MeOH}) . \mathrm{IR}(\mathrm{KBr}): 3460 m, 3400 m, 3310 \mathrm{~s}, 3290 \mathrm{~s}, 3040 m$, $2990 m, 2940 m, 1715 s, 1665 s, 1650 s, 1600 s, 1540 s, 1520 s, 1470 m, 1450 m, 1425 m, 1410 m$, 1365m, 1310m, 1290m, 1280m, 1250m, 1215m, 1170m, 695m. ${ }^{1} \mathrm{H}-\mathrm{NMR}$ ((D $)$ DMSO): 8.53 $(s, \mathrm{NH}) ; 7.55-7.3(m, 5$ arom. $\mathrm{H}, 2 \mathrm{NH}) ; 7.17(s, \mathrm{NH}) ; 5.04,5.00\left(A B, J_{\mathrm{AB}}=12.6, \mathrm{PhCH}_{2} \mathrm{O}\right)$; $4.09\left(t, J=7.8, \mathrm{CH}_{2}(2)(\right.$ Pro $\left.)\right) ; 3.85-3.7,3.7-3.55,3.55-3.54$ (3m, 1:2:1, $\mathrm{CH}_{2}$ (Gly), $\mathrm{CH}_{2}(5)\left(\right.$ Pro)); 2.15-2.0, 1.95-1.7, 1.65-1.45 (3m, 1:2:1, $\mathrm{CH}_{2}(3), \mathrm{CH}_{2}(4)($ Pro)); 1.37, 1.34, 1.32, 1.29 (4s, 1:2:2:1, $\left.3 \mathrm{Me}_{2} \mathrm{C}\right) .{ }^{13} \mathrm{C}-\mathrm{NMR}\left(\left(\mathrm{D}_{6}\right) \mathrm{DMSO}\right): 175.4,173.4,172.3,171.1,168.9$ (5s, 4 CO(amide), $\mathrm{COOH}) ; 156.5$ ( $s, \mathrm{CO}$ (urethane)); 136.9 ( $s, 1$ arom. C); 128.2, 127.7, 127.6 (3d, 5 arom. $\mathrm{CH}) ; 65.4\left(t, \mathrm{PhCH}_{2} \mathrm{O}\right) ; 62.8$ (d, $\left.\mathrm{CH}(2)(\mathrm{Pro})\right) ; 55.6,54.6$ (2s, $\left.3 \mathrm{C}(2)(\mathrm{Aib})\right) ; 47.8$ ( $t, \mathrm{CH}_{2}(5)$ (Pro)); 42.7 ( $\left.t, \mathrm{CH}_{2}(\mathrm{Gly})\right) ; 28.2,25.5$ (2t, $\mathrm{CH}_{2}(3), \mathrm{CH}_{2}(4)($ Pro)); 25.9, 25.4, 24.9, 24.1, 24.0, $23.9\left(6 q, \quad 3 \quad \mathrm{Me}_{2} \mathrm{C}\right)$. ESI-MS: $584\left(100,[M+\mathrm{Na}]^{+}\right)$. Anal. calc. for $\mathrm{C}_{27} \mathrm{H}_{39} \mathrm{~N}_{5} \mathrm{O}_{8} \cdot 1.5 \mathrm{H}_{2} \mathrm{O}$ (588.66): C 55.09, H 7.19, N 11.90; found: C 54.99, H 7.23, N 11.88.

Suitable crystals for the X-ray crystal-structure determination were grown from $\mathrm{MeOH}$.

3.6. Z-Gly-(R,S)-Phe(2Me)-Gly-Aib-Phe-OMe (8e). 3.6.1. Z-Gly-Aib-Phe-OMe. According to GP 5, a mixture of Z-Gly-Aib-OH (3) [25] (1.03 g, $3.51 \mathrm{mmol})$ and H-Phe$\mathrm{OMe} \cdot \mathrm{HCl}(834 \mathrm{mg}, 3.87 \mathrm{mmol})$ in $\mathrm{CH}_{2} \mathrm{Cl}_{2}(5 \mathrm{ml})$ was treated with PyBOP (1.68 g, 3.52 mmol) and DIEA (1.34 g, $10.65 \mathrm{mmol})$. CC (AcOEt/hexane 3:1) gave $1.36 \mathrm{~g} \mathrm{(85 \% )} \mathrm{Z-Gly-}$ Aib-Phe-OMe. Colorless solid. M.p. $150-158.7^{\circ} .[\alpha]_{\mathrm{D}}=+6.6(\mathrm{c}=1.01, \mathrm{EtOH}) . \mathrm{IR}(\mathrm{KBr})$ : $3380 m, 3260 m, 3070 m, 1745 s, 1710 s, 1680 s, 1635 s, 1555 s, 1525 m, 1380 m, 1275 m, 1250 m$, 1215m, 1205m, 1180m, 1160m, 1110w, 1080w, 1030w, 695m. ${ }^{1} \mathrm{H}-\mathrm{NMR}\left(\mathrm{CDCl}_{3}\right): 7.35-7.2$ ( $m, 8$ arom. H); 7.15-7.1 (m, 2 arom. H); 6.67 (br. $d, J=6.7, \mathrm{NH}) ; 6.54,5.37$ (2br. $s, 2 \mathrm{NH})$; 
$5.13\left(s, \mathrm{PhCH}_{2} \mathrm{O}\right) ; 4.9-4.8(A B X, \mathrm{CH}(2)(\mathrm{Phe})) ; 3.78\left(d, J=5.6, \mathrm{CH}_{2}(\mathrm{Gly})\right) ; 3.72(s, \mathrm{MeO})$; 3.2-3.05 $\left(A B X, J_{\mathrm{AB}}=13.9, \mathrm{PhCH}_{2}\right) ; 1.48\left(s, \mathrm{Me}_{2} \mathrm{C}\right) .{ }^{13} \mathrm{C}-\mathrm{NMR}\left(\mathrm{CDCl}_{3}\right): 173.7,171.9,168.6$ (3s, 2 CO(amide), COOMe); 156.7 ( $s, \mathrm{CO}$ (urethane)); 136.1, 135.9 (2s, 2 arom. C); 129.3, 128.52, 128.48, 128.2, 128.0, $127.0(6 d, 10$ arom. $\mathrm{CH}) ; 67.2\left(t, \mathrm{PhCH}_{2} \mathrm{O}\right) ; 57.2(s, \mathrm{C}(2)(\mathrm{Aib}))$; 53.4 (q, MeO); 52.3 (d, $\mathrm{CH}(2)(\mathrm{Phe})) ; 44.9$ ( $\left.t, \mathrm{CH}_{2}(\mathrm{Gly})\right) ; 37.7\left(t, \mathrm{PhCH}_{2}\right) ; 25.0,24.9$ (2q, $\left.M e_{2} \mathrm{C}\right)$. ESI-MS: $478\left(7,[M+\mathrm{Na}]^{+}\right)$. Anal. calc. for $\mathrm{C}_{24} \mathrm{H}_{29} \mathrm{~N}_{3} \mathrm{O}_{6}(455.50)$ : C 63.29, H 6.42, N 9.23; found: C 62.99, H 6.64, N 8.99.

3.6.2. H-Gly-Aib-Phe-OMe (12). According to GP 3, Z-Gly-Aib-Phe-OMe (158.6 mg, $0.348 \mathrm{mmol})$ was deprotected: $110 \mathrm{mg}(98 \%)$ of 12. Colorless oil. IR $\left(\mathrm{CDCl}_{3}\right)$ : $3420 \mathrm{~m}$, $3310 m, 2930 m, 1740 m, 1685 s, 1660 s, 1635 s, 1600 m, 1540 s, 1510 s, 1495 s, 1450 m, 1435 m$, 1385m, 1360m, 1330m, 1275m, 1175m, 690m. ${ }^{1} \mathrm{H}-\mathrm{NMR}\left(\mathrm{CDCl}_{3}\right): 7.64(s, \mathrm{NH}) ; 7.3-7.1(m, 5$ arom. H, $1 \mathrm{NH}) ; 4.9-4.8(A B X, \mathrm{CH}(2)(\mathrm{Phe})) ; 3.71(s, \mathrm{MeO}) ; 3.26$ ( $\left.s, \mathrm{CH}_{2}(\mathrm{Gly})\right) ; 3.2-3.05$ $\left(A B X, J_{\mathrm{AB}}=13.9, \mathrm{PhCH}_{2}\right) ; 1.51,1.49\left(2 s, \mathrm{Me}_{2} \mathrm{C}\right) .{ }^{13} \mathrm{C}-\mathrm{NMR}\left(\mathrm{CDCl}_{3}\right): 174.0,172.8,172.0(3 s$, $2 \mathrm{CO}$ (amide), COOMe); 136.0 ( $s, 1$ arom. C); 129.3, 128.3, 126.9 (3d, 5 arom. $\mathrm{CH}) ; 56.8$ ( $s$, $\mathrm{C}(2)(\mathrm{Aib})) ; 53.3(d, \mathrm{CH}(2)(\mathrm{Phe})) ; 52.2(q, \mathrm{MeO}) ; 44.9\left(t, \mathrm{CH}_{2}(\mathrm{Gly})\right) ; 37.6\left(t, \mathrm{PhCH}_{2}\right) ; 25.0$ ( $q$, $\left.M e_{2} \mathrm{C}\right)$. ESI-MS: $665\left(46,[2 M+\mathrm{Na}]^{+}\right), 643\left(100[2 M+1]^{+}\right), 633\left(82,[2 M-\mathrm{MeOH}+\mathrm{Na}]^{+}\right), 611$ $\left(26,[2 M-\mathrm{OMe}]^{+}\right), 344\left(88,[M+\mathrm{Na}]^{+}\right), 322\left(21,[M+1]^{+}\right)$.

3.6.3. Z-Gly-(R,S)-Phe(2Me)-Gly-Aib-Phe-OMe (8e). According to GP 5, a mixture of Z-Gly-(R,S)-Phe(2Me)-OH (11) [25a] (734 mg, $1.98 \mathrm{mmol})$ and 12 (700 mg, $2.18 \mathrm{mmol})$ in $\mathrm{CH}_{2} \mathrm{Cl}_{2}(10 \mathrm{ml})$ was treated with PyBOP $(949 \mathrm{mg}, 1.98 \mathrm{mmol})$ and DIEA (533 mg, 4.12 mmol). CC (2×, AcOEt/MeOH 20:1) and PLC (AcOEt/MeOH 10:1) gave $623 \mathrm{mg}(S, S)-8 \mathbf{e}$ and $586 \mathrm{mg}(R, S)-\mathbf{8 e}$; total yield: $1.21 \mathrm{mg}(91 \%)$.

Z-Gly-(S)-Phe(2Me)-Gly-Aib-Phe-OMe ((S,S)-8e). Colorless solid. M.p. 162.5-164.1 . $[\alpha]_{\mathrm{D}}=-69.0(\mathrm{c}=1.01, \mathrm{EtOH}) . \mathrm{IR}(\mathrm{KBr}): 3410 m, 3280 s, 1745 s, 1730 s, 1670 s, 1660 s, 1535 s$, $1455 m, 1385 m, 1360 m, 1325 m, 1280 m, 1230 s, 1190 m, 1185 m, 1165 m, 1150 m, 1110 w$ 
1080w, 700m. ${ }^{1} \mathrm{H}-\mathrm{NMR}\left(\mathrm{CDCl}_{3}\right)$ : 7.4-7.05 (m, 15 arom. H, $\left.3 \mathrm{NH}\right) ; 6.49(s, \mathrm{NH}) ; 5.80(t$-like, $\mathrm{NH}) ; 5.05,4.98\left(A B, J_{\mathrm{AB}}=12, \mathrm{PhCH}_{2} \mathrm{O}\right) ; 4.8-4.7(A B X, \mathrm{CH}(2)(\mathrm{Phe})) ; 3.9-3.65(m, 2$ $\left.\mathrm{CH}_{2}(\mathrm{Gly})\right) ; 3.60(s, \mathrm{MeO}) ; 3.29,3.03\left(A B, J_{\mathrm{AB}}=13.6, \mathrm{PhCH}_{2}\right) ; 3.2-3.05\left(m, \mathrm{PhCH}_{2}\right) ; 1.48$, 1.44, $1.40\left(3 s, \mathrm{Me}_{2} \mathrm{C}, \mathrm{Me}(\mathrm{Phe}(2 \mathrm{Me}))\right) .{ }^{13} \mathrm{C}-\mathrm{NMR}\left(\mathrm{CDCl}_{3}\right): 175.3,175.0,173.2,170.7,170.0$ (5s, 4 CO(amide), COOMe); 157.3 ( $s, \mathrm{CO}($ urethane)); 136.8, 136.1, 135.3 (3s, 3 arom. C); $130.5,129.1,128.6,128.5,128.4,128.3,128.0,127.2,126.8(9 d, 15$ arom. $\mathrm{CH}) ; 67.3(t$, $\left.\mathrm{PhCH}_{2} \mathrm{O}\right) ; 59.6,57.0(2 s, \mathrm{C}(2)(\mathrm{Aib}), \mathrm{C}(2)(\mathrm{Phe}(2 \mathrm{Me}))) ; 54.1(d, \mathrm{CH}(2)(\mathrm{Phe})) ; 52.5(q, \mathrm{MeO})$; 45.4, $44.3\left(2 t, 2 \mathrm{CH}_{2}(\mathrm{Gly})\right) ; 36.9,30.7\left(2 t, 2 \mathrm{PhCH}_{2}\right) ; 25.9,24.3,22.7$ (3q, Me $2 \mathrm{C}$, $\mathrm{Me}(\mathrm{Phe}(2 \mathrm{Me})))$. ESI-MS: $696\left(100,[M+\mathrm{Na}]^{+}\right)$. Anal. calc. for $\mathrm{C}_{36} \mathrm{H}_{43} \mathrm{~N}_{5} \mathrm{O}_{8} \cdot \mathrm{H}_{2} \mathrm{O}$ (691.78): C 62.50, H 6.56, N 10.12; found: C 62.43, H 6.30, N 10.07.

Suitable crystals for the X-ray crystal-structure determination were grown from AcOEt/hexane by slow evaporation of the solvent.

Z-Gly-(R)-Phe(2Me)-Gly-Aib-Phe-OMe $\quad((R, S)-8 \mathbf{e}) . \quad$ Colorless $\quad$ solid. M.p. 116.4$118.2^{\circ} \cdot[\alpha]_{\mathrm{D}}=+67.5(\mathrm{c}=1.05, \mathrm{EtOH}) . \mathrm{IR}(\mathrm{KBr}): 3380 m, 3280 s, 1730 s, 1680 s, 1670 s, 1660 s$, $1540 s, 1530 s, 1455 m, 1440 m, 1410 m, 1390 m, 1365 m, 1345 m, 1310 m, 1275 m, 1230 m, 1195 m$, 1175m, 1150m, 1120w, 1080w, 1045m, 700m. ${ }^{1} \mathrm{H}-\mathrm{NMR}\left(\mathrm{CDCl}_{3}\right): 8.35$ (br. $\left.s, \mathrm{NH}\right) ; 7.65$ ( $s$, $\mathrm{NH}) ; 7.42(d, J=7.9, \mathrm{NH}) ; 7.4-7.05(m, 15$ arom. $\mathrm{H}) ; 7.00(s, \mathrm{NH}) ; 6.18$ (br. $s, \mathrm{NH}) ; 5.12$, $5.00\left(A B, J_{\mathrm{AB}}=12.3, \mathrm{PhCH}_{2} \mathrm{O}\right) ; 4.65-4.55(m, \mathrm{CH}(2)(\mathrm{Phe})) ; 4.0-3.6\left(m, 2 \mathrm{CH}_{2}(\mathrm{Gly})\right) ; 3.47$, $3.10\left(A B, J_{\mathrm{AB}}=13.7, \mathrm{PhCH}_{2}\right) ; 3.24(s, \mathrm{MeO}) ; 3.25-2.95\left(m, \mathrm{PhCH}_{2}\right) ; 1.43,1.38,1.26(3 s$, $\left.\mathrm{Me}_{2} \mathrm{C}, \mathrm{Me}(\mathrm{Phe}(2 \mathrm{Me}))\right) .{ }^{13} \mathrm{C}-\mathrm{NMR} \quad\left(\mathrm{CDCl}_{3}\right): 176.9, \quad 176.4,172.3,171.0,169.9$ (5s, 4 $\mathrm{CO}$ (amide), COOMe); 157.0 ( $s, \mathrm{CO}$ (urethane)); 136.7, 136.4, 136.2 (3s, 3 arom. C); 131.1, 129.0, 128.5, 128.4, 128.2, 128.1, 126.8, 126.6 (8d, 15 arom. $\mathrm{CH}) ; 67.1\left(t, \mathrm{PhCH}_{2} \mathrm{O}\right) ; 59.4$, $57.0(2 s, \mathrm{C}(2)(\mathrm{Aib}), \mathrm{C}(2)(\mathrm{Phe}(2 \mathrm{Me}))) ; 54.1(d, \mathrm{CH}(2)(\mathrm{Phe})) ; 52.0(q, \mathrm{MeO}) ; 45.5,44.3(2 t, 2$ $\left.\mathrm{CH}_{2}(\mathrm{Gly})\right) ; 39.5,37.2\left(2 t, 2 \mathrm{PhCH}_{2}\right) ; 27.0,23.5$ (2q, 1:2, $\left.\mathrm{Me}_{2} \mathrm{C}, \mathrm{Me}(\mathrm{Phe}(2 \mathrm{Me}))\right)$. ESI-MS: 696 
$\left(100,[M+\mathrm{Na}]^{+}\right)$. Anal. calc. for $\mathrm{C}_{36} \mathrm{H}_{43} \mathrm{~N}_{5} \mathrm{O}_{8}$ (673.76): C 64.18, H 6.43, N 10.39; found: C 63.91, H 6.33, N 10.53.

3.7. Z-Gly-(R,S)-Phe(2Me)-Gly-Aib-Aib-N(Me)Ph (8f). According to GP 5, a mixture of 11 (100 mg, $0.270 \mathrm{mmol})$ and H-Gly-Aib-Aib-N(Me)Ph [25a] (99 mg, $0.296 \mathrm{mmol})$ in $\mathrm{CH}_{2} \mathrm{Cl}_{2}(2 \mathrm{ml})$ was treated with PyBOP $(129 \mathrm{mg}, 0.270 \mathrm{mmol})$ and DIEA (85 mg, 0.658 mmol): $152 \mathrm{mg}$ (82\%) of 8f. Colorless solid. M.p. 191.9-192.8 . IR (KBr): 3410m, 3300m, $1725 m, 1680 s, 1665 s, 1650 s, 1640 s, 1595 m, 1535 s, 1495 m, 1455 m, 1395 m, 1375 m, 1365 m$, 1270m, 1250m, 1240m, 1095m, 710m. ${ }^{1} \mathrm{H}-\mathrm{NMR}\left(\mathrm{CDCl}_{3}\right): 7.86$ (br. $\left.s, \mathrm{NH}\right) ; 7.4-7.05$ ( $m, 15$ arom. H, $2 \mathrm{NH}) ; 6.83,6.54$ (2br. $s, 2 \mathrm{NH}) ; 5.08,5.02\left(A B, J_{\mathrm{AB}}=12.2, \mathrm{PhCH}_{2} \mathrm{O}\right) ; 3.8-3.6(m$, $\left.2 \mathrm{CH}_{2}(\mathrm{Gly})\right) ; 3.23(s, \mathrm{MeN}) ; 3.23,2.98\left(A B, J_{\mathrm{AB}}=13.6, \mathrm{PhCH}_{2}\right) ; 1.49,1.46,1.27(3 s, 3: 1: 1,2$ $\left.\mathrm{Me}_{2} \mathrm{C}, \mathrm{Me}(\mathrm{Phe}(2 \mathrm{Me}))\right) .{ }^{13} \mathrm{C}-\mathrm{NMR} \quad\left(\mathrm{CDCl}_{3}\right): 175.6,175.2,174.0,170.8,169.2 \quad(5 s, 5$ $\mathrm{CO}$ (amide)); 157.3 ( $s, \mathrm{CO}($ urethane)); 145.3, 136.2, 135.6 (3s, 3 arom. C); 130.5, 129.1, 128.4, 128.2, 128.1, 127.9, 127.0, 126.9 (8d, 15 arom. $\mathrm{CH}) ; 67.0\left(t, \mathrm{PhCH}_{2} \mathrm{O}\right) ; 59.3,57.1(2 s$, $2 \mathrm{C}(2)(\mathrm{Aib}), \mathrm{C}(2)(\mathrm{Phe}(2 \mathrm{Me}))) ; 45.4,44.8,40.7$ (3t, $\left.2 \mathrm{CH}_{2}(\mathrm{Gly}), \mathrm{PhCH}_{2}\right) ; 40.3$ (q, MeN); 25.91, 25.88, 24.9, $22.7\left(4 q, 1: 2: 1: 1,2 \mathrm{Me}_{2} \mathrm{C}, \mathrm{Me}(\mathrm{Phe}(2 \mathrm{Me}))\right)$. ESI-MS: $725\left(8,[M+\mathrm{K}]^{+}\right), 709$ $\left(100,[M+\mathrm{Na}]^{+}\right), 580\left(5,[M-\mathrm{N}(\mathrm{Me}) \mathrm{Ph}]^{+}\right)$. Anal. calc. for $\mathrm{C}_{37} \mathrm{H}_{46} \mathrm{~N}_{6} \mathrm{O}_{7}(686.81): \mathrm{C}$ 64.71, $\mathrm{H}$ 6.75, N 12.24; found: C 64.90, H 6.89, N 12.40.

Suitable crystals for the X-ray crystal-structure determination were grown from AcOEt/hexane by slow evaporation of the solvent.

3.8. Z-Gly-(R,S)-Phe(2Me)-Pro-Aib-Phe-OMe (8g). 3.8.1. Z-Pro-Aib-Phe-OMe. According to GP 5, a mixture of Z-Pro-Aib-OH [25b] (1.14 g, $3.40 \mathrm{mmol})$ and H-Phe$\mathrm{OMe} \cdot \mathrm{HCl}(808 \mathrm{mg}, 3.75 \mathrm{mmol})$ in $\mathrm{CH}_{2} \mathrm{Cl}_{2}(6 \mathrm{ml})$ was treated with PyBOP $(1.63 \mathrm{~g}, 3.40$ mmol) and DIEA (1.32 g, $10.23 \mathrm{mmol}) . \mathrm{CC}$ (AcOEt/hexane 3:1) gave $1.33 \mathrm{~g}$ (79\%) Z-ProAib-Phe-OMe. Colorless solid. M.p. $169.7-170.4^{\circ} .[\alpha]_{\mathrm{D}}=-23.8(\mathrm{c}=0.817, \mathrm{EtOH}) . \mathrm{IR}(\mathrm{KBr})$ : $3380 m, 3280 m, 1745 s, 1690 s, 1665 s, 1640 s, 1550 m, 1525 m, 1455 m, 1435 s, 1355 m, 1240 m$, 
1215m, 1205m, 1180m, 1170m, 1120m, 700m. ${ }^{1} \mathrm{H}-\mathrm{NMR}\left(\mathrm{CDCl}_{3}\right): 7.35-7.1$ (m, 10 arom. H, $0.8 \mathrm{NH}) ; 6.86$ (br. $s, \mathrm{NH}) ; 6.40$ (br. $s, 0.2 \mathrm{NH}) ;$ 5.2-5.1 ( $\left.m, \mathrm{PhCH}_{2} \mathrm{O}\right) ; 4.8-4.75,4.25-4.2(2 m$, $\mathrm{CH}(2)(\mathrm{Phe}), \mathrm{CH}(2)(\mathrm{Pro})) ; 3.68(s, \mathrm{MeO}) ; 3.6-3.4\left(m, \mathrm{CH}_{2}(5)(\mathrm{Pro})\right) ; 3.2-3.0\left(A B X, J_{\mathrm{AB}}=13.9\right.$, $\left.\mathrm{PhCH}_{2}\right) ; 2.25-1.25\left(m, \mathrm{CH}_{2}(3), \mathrm{CH}_{2}(4)(\mathrm{Pro})\right) ; 1.44,1.26$ (2br. $\left.s, \mathrm{Me}_{2} \mathrm{C}\right) .{ }^{13} \mathrm{C}-\mathrm{NMR}\left(\mathrm{CDCl}_{3}\right)$ : 173.7, 171.9 (2s, $2 \mathrm{CO}$ (amide), COOMe); 156.1 ( $s, \mathrm{CO}$ (urethane)); 136.3 (s, 2 arom. C); 129.2, 128.4, 128.3, 128.1, 127.8, $126.8(6 d, 10$ arom. $\mathrm{CH}) ; 67.2\left(t, \mathrm{PhCH}_{2} \mathrm{O}\right) ; 61.0(d$, $\mathrm{CH}(2)(\mathrm{Pro})) ; 57.2(s, \mathrm{C}(2)(\mathrm{Aib})) ; 53.6(d, \mathrm{CH}(2)(\mathrm{Phe})) ; 52.0(s, \mathrm{MeO}) ; 47.0\left(t, \mathrm{CH}_{2}(5)\right.$ (Pro)); $37.8\left(t, \mathrm{PhCH}_{2}\right) ; 28.4,24.8\left(2 t, \mathrm{CH}_{2}(3), \mathrm{CH}_{2}(4)\right.$ (Pro) $) ; 25.5,24.8$ (2q, Me 2 C). ESI-MS: 518 $\left(100,[M+N a]^{+}\right)$. Anal. calc. for $\mathrm{C}_{27} \mathrm{H}_{33} \mathrm{~N}_{3} \mathrm{O}_{6}$ (495.57): C 65.44, H 6.71, N 8.48; found: C 65.49, H 6.97, N 8.54.

3.8.2. H-Pro-Aib-Phe-OMe (13). According to GP 3, Z-Pro-Aib-Phe-OMe (1.15 g, $2.32 \mathrm{mmol})$ in $\mathrm{MeOH}(25 \mathrm{ml})$ in the presence of $\mathrm{Pd} / \mathrm{C}(113 \mathrm{mg})$ was deprotected $(2 \mathrm{~h}): 842$ mg $(84 \%)$ 13. Colorless oil. $[\alpha]_{\mathrm{D}}=+3.6\left(\mathrm{c}=1.24, \mathrm{CHCl}_{3}\right)$. IR $\left(\mathrm{CHCl}_{3}\right): 3280 m, 3020 m$, $3000 s, 2980 m, 1740 s, 1670 s, 1510 s, 1455 m, 1440 m, 1390 m, 1360 m, 1215 m, 1180 m, 850 m$, 700m. ${ }^{1} \mathrm{H}-\mathrm{NMR}\left(\mathrm{CDCl}_{3}\right): 7.89(s, \mathrm{NH}) ; 7.41(d, J=7.5, \mathrm{NH}) ; 7.3-7.15(m, 5$ arom. $\mathrm{H}) ; 4.8-$ 4.75, 3.7-3.65 (2m, $\mathrm{CH}(2)(\mathrm{Pro}), \mathrm{CH}(2)(\mathrm{Phe})) ; 3.69(s, \mathrm{MeO}) ; 3.2-2.9\left(m, \mathrm{CH}_{2}(5)(\mathrm{Pro})\right.$, $\mathrm{PhCH}_{2}, \mathrm{NH}(\mathrm{Pro})$ ); 2.15-2.05, 1.9-1.85, 1.75-1.65 (3m, 1:1:2, $\mathrm{CH}_{2}(3), \mathrm{CH}_{2}$ (4)(Pro)); 1.47, $1.45\left(2 s, \mathrm{Me}_{2} \mathrm{C}\right) .{ }^{13} \mathrm{C}-\mathrm{NMR}\left(\mathrm{CDCl}_{3}\right): 175.2,174.2,172.1$ (3s, $2 \mathrm{CO}$ (amide), COOMe); 136.3 $(s, 1$ arom. C); 129.3, 128.4, 126.9 (3d, 5 arom. $\mathrm{CH}) ; 60.7$ (d, $\mathrm{CH}(2)(\mathrm{Pro})) ; 56.9$ (s, $\mathrm{C}(2)$ (Aib)); $53.5(d, \mathrm{CH}(2)(\mathrm{Phe})) ; 52.2(s, \mathrm{MeO}) ; 47.2\left(t, \mathrm{CH}_{2}(5)(\mathrm{Pro})\right) ; 37.7\left(t, \mathrm{PhCH}_{2}\right)$; 30.6, 26.0 (2t, $\left.\mathrm{CH}_{2}(3), \mathrm{CH}_{2}(4)(\mathrm{Pro})\right) ; 25.2,25.1$ (2q, Me 2 C). CI-MS: 363 (21), 362 (100, $\left.[M+1]^{+}\right)$

3.8.3. Z-Gly-(R,S)-Phe(2Me)-Pro-Aib-Phe-OMe (8g). According to GP 5, a mixture of $11(374.5 \mathrm{mg}, 1.01 \mathrm{mmol})$ and $\mathbf{1 3}(400 \mathrm{mg}, 1.11 \mathrm{mmol})$ in $\mathrm{CH}_{2} \mathrm{Cl}_{2}(10 \mathrm{ml})$ was treated with TBTU (326.2 mg, $1.02 \mathrm{mmol})$ and DIEA (301.8 $\mathrm{mg}, 2.34 \mathrm{mmol})$ for $16 \mathrm{~h} . \mathrm{CC}(4 \times$, 
AcOEt/MeOH 20:1) gave $193.9 \mathrm{mg}(S, S)-\mathbf{8 g}$ and $319.8 \mathrm{mg}(R, S)-\mathbf{8 g}$; total yield: $513.7 \mathrm{mg}$ $(71 \%)$.

Z-Gly-(S)-Phe(2Me)-Pro-Aib-Phe-OMe ((S,S,S)-8g). Colorless lacquer. M.p. 59.1$60.7^{\circ} .[\alpha]_{\mathrm{D}}=-17.6(\mathrm{c}=1.03, \mathrm{EtOH}) . \mathrm{IR}(\mathrm{KBr}): 3300 m, 2940 m, 1745 s, 1740 s, 1730 s, 1720 s$, $1670 s, 1660 s, 1635 s, 1625 s, 1565 m, 1540 s, 1520 s, 1505 s, 1460 m, 1455 m, 1410 m, 1385 m$, $1240 s, 1170 m, 1050 m, 700 m .{ }^{1} \mathrm{H}-\mathrm{NMR}\left(\mathrm{CDCl}_{3}\right): 7.49(d, J=8.1, \mathrm{NH}) ; 7.45-7.05(m, 15$ arom. $\mathrm{H}, \mathrm{NH}) ; 6.92(s, \mathrm{NH}) ; 5.71(t$-like, $\mathrm{NH}) ; 5.16,5.10\left(A B, J_{\mathrm{AB}}=12.2, \mathrm{PhCH}_{2} \mathrm{O}\right) ; 4.8-4.7$ ( $m, \mathrm{CH}(2)(\mathrm{Phe})) ; 4.41$ ( $t, \mathrm{CH}(2)(\mathrm{Pro})) ; 3.9-3.7$ ( $A B$ of $\left.A B X, \mathrm{CH}_{2}(\mathrm{Gly})\right) ; 3.61(s, \mathrm{MeO}) ; 3.4-$ 3.1, 3.1-2.95 (2m, 1:1, $\left.\left.2 \mathrm{PhCH}_{2}\right), \mathrm{CH}_{2}(5)(\mathrm{Pro})\right)$; 2.25-2.1, 1.85-1.65, 1.65-1.5 (3m, 1:2:1, $\mathrm{CH}_{2}(3), \mathrm{CH}_{2}$ (4)(Pro)); 1.52, 1.50, 1.34 (3s, $\mathrm{Me}_{2} \mathrm{C}, \mathrm{Me}(\mathrm{Phe}(2 \mathrm{Me}))$ ). ${ }^{13} \mathrm{C}-\mathrm{NMR}\left(\mathrm{CDCl}_{3}\right):$ 175.1, 172.2, 171.6, 171.1, 169.0 (5s, $4 \mathrm{CO}$ (amide), COOMe); 156.8 ( $s, \mathrm{CO}$ (urethane)); 137.1, 136.0, 134.5 (3s, 3 arom. C); 130.2, 129.2, 128.9, 128.6, 128.5, 128.2, 127.7, 126.5 (8d, 15 arom. $\mathrm{CH}) ; 67.4 \quad\left(t, \quad \mathrm{PhCH}_{2} \mathrm{O}\right) ; 63.6 \quad(d, \quad \mathrm{C}(2)(\mathrm{Pro})) ; 60.1,57.1 \quad(2 s, \mathrm{C}(2)$ (Aib), $\mathrm{C}(2)(\mathrm{Phe}(2 \mathrm{Me}))) ; 53.9(d, \mathrm{CH}(2)(\mathrm{Phe})) ; 52.0(q, \mathrm{MeO}) ; 48.6\left(t, \mathrm{CH}_{2}(5)(\mathrm{Pro})\right) ; 44.8(t$, $\mathrm{CH}_{2}$ (Gly)); 43.0, 37.9 (2t, $\left.2 \mathrm{PhCH}_{2}\right)$; 28.3, 26.0 (2t, $\left.\mathrm{CH}_{2}(3), \mathrm{CH}_{2}(4)(\mathrm{Pro})\right) ; 26.7,24.4,24.0$ $\left(3 q, M e_{2} \mathrm{C}, \mathrm{Me}(\mathrm{Phe}(2 \mathrm{Me}))\right)$. ESI-MS: $736\left(100,[M+\mathrm{Na}]^{+}\right)$.

Z-Gly-(R)-Phe(2Me)-Pro-Aib-Phe-OMe ((R,S,S)-8g). Colorless solid. M.p. 165.5$166.5^{\circ} .[\alpha]_{\mathrm{D}}=+68.9(\mathrm{c}=1.04, \mathrm{EtOH}) . \mathrm{IR}(\mathrm{KBr}): 3400 s, 3060 m, 3030 m, 2980 m, 2940 m$, $1750 s, 1730 s, 1655 s, 1625 s, 1545 s, 1535 s, 1500 m, 1455 m, 1445 m, 1405 m, 1385 m, 1375 m$, $1365 m, 1305 m, 1280 m, 1240 s, 1170 m, 1160 m, 1130 w, 1100 m, 1080 m, 1045 m, 1030 m$, 1000m, 740m, 700m. ${ }^{1} \mathrm{H}-\mathrm{NMR}\left(\mathrm{CDCl}_{3}\right): 7.50(d, J=8.1, \mathrm{NH}) ; 7.39(s, \mathrm{NH}) ; 7.4-7.0(m, 15$ arom. H); 6.85 ( $s, \mathrm{NH}) ; 5.70$ (br. $s, \mathrm{NH}) ; 5.08\left(s, \mathrm{PhCH}_{2} \mathrm{O}\right) ; 4.75-4.65(m, \mathrm{CH}(2)(\mathrm{Phe})) ; 4.37$ (t-like, $\mathrm{CH}(2)(\mathrm{Pro})) ; 4.0-3.8$ ( $A B$ of $\left.A B X, \mathrm{CH}_{2}(\mathrm{Gly})\right)$; 3.75-3.65, 3.35-3.2 (2m, $\mathrm{CH}_{2}$ (5)(Pro)); $3.61(s, \mathrm{MeO}) ; 3.42,3.17\left(\mathrm{AB}, J_{\mathrm{AB}}=13.8, \mathrm{PhCH}_{2}\right) ; 3.25-3.1,3.1-2.95\left(2 m, \mathrm{PhCH}_{2}\right) ; 2.2-$ 2.05, 1.95-1.8, 1.8-1.6 (3m, 1:1:2, $\left.\mathrm{CH}_{2}(3), \mathrm{CH}_{2}(4)(\mathrm{Pro})\right) ; 1.46,1.39,1.26\left(3 s, \mathrm{Me}_{2} \mathrm{C}\right.$, 
$\mathrm{Me}(\mathrm{Phe}(2 \mathrm{Me}))) .{ }^{13} \mathrm{C}-\mathrm{NMR}\left(\mathrm{CDCl}_{3}\right): 175.3,172.5,172.4,171.2,169.3$ (5s, $4 \mathrm{CO}$ (amide), COOMe); 156.8 (s, CO(urethane)); 136.8, 136.6, 136.1 (3s, 3 arom. C); 131.1, 129.1, 128.6, 128.3, 128.22, 128.17, 128.1, 126.8, 126.6 (9d, 15 arom. $\mathrm{CH}) ; 67.2\left(t, \mathrm{PhCH}_{2} \mathrm{O}\right) ; 63.7(d$, $\mathrm{CH}(2)(\mathrm{Pro})) ; 59.1,57.1$ (2s, C(2)(Aib), $\mathrm{C}(2)(\mathrm{Phe}(2 \mathrm{Me}))) ; 53.6$ (d, $\mathrm{CH}(2)(\mathrm{Phe})) ; 52.0$ ( $q$, $\mathrm{MeO}) ; 48.7\left(t, \mathrm{CH}_{2}(5)(\mathrm{Pro})\right) ; 44.4\left(t, \mathrm{CH}_{2}(\mathrm{Gly})\right) ; 40.9,37.8\left(2 t, 2 \mathrm{PhCH}_{2}\right) ; 28.5,26.0$ (2t, $\left.\mathrm{CH}_{2}(3), \mathrm{CH}_{2}(4)(\mathrm{Pro})\right) ; 27.0,24.1,21.1$ (3q, Me $\left.2 \mathrm{C}, \mathrm{Me}(\mathrm{Phe}(2 \mathrm{Me}))\right)$. ESI-MS: 736 (100, $\left.[M+\mathrm{Na}]^{+}\right), 714\left(10,[M+1]^{+}\right), 535\left(6,[M-\mathrm{PheOMe}]^{+}\right)$.

Suitable crystals for the X-ray crystal-structure determination were grown from $\mathrm{AcOEt} / \mathrm{MeOH}$ by slow evaporation of the solvent.

3.9. Z-Gly-(R,S)-Phe(2Me)-Pro-Aib-Aib-N(Me)Ph (8h). According to GP 5, a mixture of $11(1.25 \mathrm{~g}, 3.36 \mathrm{mmol})$ and H-Pro-Aib-Aib-N(Me)Ph [25b] (1.40 g, $3.73 \mathrm{mmol})$ in $\mathrm{CH}_{2} \mathrm{Cl}_{2}$ (30 ml) was treated with TBTU (1.20 g, $3.74 \mathrm{mmol}), \mathrm{HOBt}(910 \mathrm{mg}, 6.73 \mathrm{mmol})$, and DIEA (916 mg, $7.09 \mathrm{mmol})$ for 18 h. $\mathrm{CC}\left(\mathrm{CH}_{2} \mathrm{Cl}_{2} / \mathrm{MeOH} 15: 1\right)$ gave $1.76 \mathrm{~g}$ (72\%) 8h. Separation of 815.5 mg of the mixture of the diastereoisomers by PLC (AcOEt/MeOH 20:1, 5× developed) led to $224.6 \mathrm{mg}$ of $(S, S)-8 \mathbf{h}$ and $470.6 \mathrm{mg}$ of $(R, R)-\mathbf{8 h}$.

Z-Gly-(S)-Phe(2Me)-Pro-Aib-Aib-N(Me)Ph ((S,S)-8h). Colorless solid. M.p. 92.493.9 $.[\alpha]_{\mathrm{D}}=+14.0(\mathrm{c}=0.57, \mathrm{EtOH}) . \mathrm{IR}(\mathrm{KBr}): 3310 \mathrm{~s}, 2980 \mathrm{~m}, 2930 \mathrm{~m}, 1730 \mathrm{~s}, 1680 \mathrm{~s}, 1660 \mathrm{~s}$, $1630 s, 1595 m, 1545 s, 1540 s, 1530 s, 1495 s, 1465 m, 1455 m, 1405 m, 1390 s, 1360 m, 1280 m$, $1240 s, 1200 m, 1160 m, 1090 m, 1050 m, 740 m, 700 s .{ }^{1} \mathrm{H}-\mathrm{NMR}\left(\mathrm{CDCl}_{3}\right): 7.52(s, \mathrm{NH}) ; 7.35-$ $7.05(m, 15$ arom. $\mathrm{H}, 2 \mathrm{NH}) ; 6.29$ (br. $s, \mathrm{NH}) ; 5.12,5.08\left(A B, J_{\mathrm{AB}}=12.3, \mathrm{PhCH}_{2} \mathrm{O}\right) ; 4.22(t, J$ $=8.3, \mathrm{CH}(2)(\mathrm{Pro})) ; 3.8-3.55\left(A B\right.$ of $\left.A B X, J_{\mathrm{AB}}=17.2, \mathrm{CH}_{2}(\mathrm{Gly})\right) ; 3.38(\mathrm{~s}, \mathrm{MeN}) ; 3.2-3.0(m$, $\mathrm{CH}_{2}$ (5)(Pro)); $2.91\left(s, \mathrm{PhCH}_{2}\right) ; 2.3-2.15,1.85-1.7,1.7-1.4\left(3 m, 1: 1: 2, \mathrm{CH}_{2}(3), \mathrm{CH}_{2}\right.$ (4)(Pro)); 1.60, 1.57, 1.47, 1.45, $1.40\left(5 s, 2 \mathrm{Me}_{2} \mathrm{C}, \mathrm{Me}(\mathrm{Phe}(2 \mathrm{Me}))\right) .{ }^{13} \mathrm{C}-\mathrm{NMR}\left(\mathrm{CDCl}_{3}\right): 174.7,174.1$, 171.8, 171.6, 169.8 (5s, $5 \mathrm{CO}$ (amide)); 157. ( $s, \mathrm{CO}($ urethane)); 146.1, 136.4, $134.6(3 s, 3$ arom. C); 130.1, 129.0, 128.8, 128.6, 128.3, 128.1, 127.6, 127.2, 126.6 (9d, 15 arom. CH); 
$67.0\left(t, \mathrm{PhCH}_{2} \mathrm{O}\right) ; 64.4$ (d, C(2)(Pro)); 60.3, 57.2, 57.0 (3s, 2 C(2)(Aib), C(2)(Phe(2Me))); 48.6, 44.6, 43.6 (3t, $\mathrm{CH}_{2}$ (Gly), $\left.\mathrm{CH}_{2}(5)(\mathrm{Pro}), \mathrm{PhCH}_{2}\right)$; 40.1 (q, MeN); 28.6, $26.2\left(2 t, \mathrm{CH}_{2}(3)\right.$, $\mathrm{CH}_{2}(4)\left(\right.$ Pro)); 27.8, 26.4, 25.4, 24.3, 23.7 (5q, $2 \mathrm{Me}_{2} \mathrm{C}$, Me(Phe(2Me))). ESI-MS: 749 (100, $[M+\mathrm{Na}]^{+}$). Anal. calc. for $\mathrm{C}_{40} \mathrm{H}_{50} \mathrm{~N}_{6} \mathrm{O}_{7} \cdot 0.5 \mathrm{H}_{2} \mathrm{O}$ (735.88): C 65.29, H 6.99, N 11.42; found: $\mathrm{C}$ 65.28, H 7.17, N 11.15.

Z-Gly-(R)-Phe(2Me)-Pro-Aib-Aib-N(Me)Ph ((R,S)-8h). Colorless solid. M.p. 168.7$170.2^{\circ} .[\alpha]_{\mathrm{D}}=+114(\mathrm{c}=0.51, \mathrm{EtOH}) . \mathrm{IR}(\mathrm{KBr}): 3300 s, 3060 w, 3030 w, 2980 w, 2940 w$, $1725 s, 1660 s, 1625 s, 1595 m, 1540 s, 1495 m, 1465 m, 1455 m, 1410 m, 1395 m, 1375 m, 1360 m$, 1280m, 1240m, 1160m, 1135m, 1090m, 1050m, 705s. ${ }^{1} \mathrm{H}-\mathrm{NMR}\left(\mathrm{CDCl}_{3}\right): 7.44(s, \mathrm{NH}) ; 7.35-$ $7.05(m, 15$ arom. $\mathrm{H}, 2 \mathrm{NH}) ; 5.95$ (br. $s, \mathrm{NH}) ; 5.09\left(s, \mathrm{PhCH}_{2} \mathrm{O}\right) ; 4.23(t, J=8.3, \mathrm{CH}(2)$ (Pro)); 3.9-3.6 $\left(A B\right.$ of $\left.A B X, J_{\mathrm{AB}}=17, \mathrm{CH}_{2}(\mathrm{Gly})\right) ; 3.7-3.6,3.3-3.1\left(2 m, \mathrm{CH}_{2}(5)(\right.$ Pro $\left.)\right) ; 3.37,3.08$ $\left(A B, J_{\mathrm{AB}}=13.7, \mathrm{PhCH}_{2}\right) ; 3.29(s, \mathrm{MeN}) ; 2.3-2.15,1.95-1.85,1.85-1.6\left(3 m, 1: 1: 2, \mathrm{CH}_{2}(3)\right.$, $\mathrm{CH}_{2}$ (4)(Pro)); 1.54, 1.45, 1.39, $1.17\left(4 s, 1: 1: 2: 1,2 \mathrm{Me}_{2} \mathrm{C}, \mathrm{Me}(\mathrm{Phe}(2 \mathrm{Me}))\right) .{ }^{13} \mathrm{C}-\mathrm{NMR}$ $\left(\mathrm{CDCl}_{3}\right): 174.8,173.9,173.1,171.3,169.6$ (5s, $5 \mathrm{CO}$ (amide)); 156.8 (s, $\mathrm{CO}$ (urethane)); 145.9, 136.5, 136.2 (3s, 3 arom. C); 131.2, 128.9, 128.6, 128.3, 128.2, 127.2, 126.8, 126.6 (8d, 15 arom. $\mathrm{CH}) ; 67.1\left(t, \quad \mathrm{PhCH}_{2} \mathrm{O}\right) ; 64.4$ (d, $\left.\mathrm{CH}(2)(\mathrm{Pro})\right) ; 58.9,57.1$ (2s, $2 \mathrm{C}(2)(\mathrm{Aib})$, $\mathrm{C}(2)(\mathrm{Phe}(2 \mathrm{Me}))) ; 48.8\left(t, \mathrm{CH}_{2}(5)(\mathrm{Pro})\right) ; 44.2\left(t, \mathrm{CH}_{2}(\mathrm{Gly})\right) ; 40.7\left(t, \mathrm{PhCH}_{2}\right) ; 40.1$ (q, MeN); 28.7, $26.2\left(2 t, \mathrm{CH}_{2}(3), \mathrm{CH}_{2}(4)(\right.$ Pro $\left.)\right) ; 27.7,26.3,25.6,23.7,20.9$ (5q, $2 \mathrm{Me}_{2} \mathrm{C}$, Me(Phe(2Me))). ESI-MS: $749\left(100,[M+\mathrm{Na}]^{+}\right), 620\left(7,[M-\mathrm{N}(\mathrm{Me}) \mathrm{Ph}]^{+}\right)$.

Suitable crystals for the X-ray crystal-structure determination were grown from $\mathrm{AcOEt} / \mathrm{MeOH} / \mathrm{hexane}$ by slow evaporation of the solvent.

4. X-Ray Crystal-Structure Determination of 6a, 8a, $(S, S)-\mathbf{8 e}, \mathbf{8 f},(R, S, S)-\mathbf{8 g},(R, S)-\mathbf{8 h}$, and 10 (see Table 4 and Figs. $1-7)^{9}$ ). The measurements were made using graphite-

$\left.{ }^{9}\right) \quad$ CCDC-989272-989278 contain the supplementary crystallographic data for this paper. These data can be obtained free of charge from The Cambridge Crystallographic Data 
monochromated $\mathrm{Mo} K_{\alpha}$ radiation $(\lambda 0.7107 \AA)$ on a Rigaku AFC5R diffractometer fitted to a 12-kW rotating-anode generator. The intensities were corrected for Lorentz and polarization effects. Empirical absorption corrections based on azimuthal scans of several reflections [45] were applied in the cases of $\mathbf{8 f}$ and $(R, S, S)-\mathbf{8 g}$. Equivalent reflections, other than Friedel pairs for structures in non-centrosymmetric space groups, were merged. The data collection and refinement parameters are given in Table 4, and views of the molecules are shown in Figs. 1 7. Each structure was solved by direct methods using SHELXS86 [46] for 6a, 8a, $(S, S)-\mathbf{8 e}$, 8f, $(R, S, S)-\mathbf{8 g}$, and 10, which revealed the positions of all non-H-atoms, and $\operatorname{SnB}[47]^{10)}$ for $(R, S)-\mathbf{8 h}$, which revealed the positions of most of the non-H-atoms. In the latter case, the remaining non-H atoms were located in a subsequent difference electron density map. For all structures, the non-H-atoms were refined anisotropically. Unless indicated otherwise fro individual structures below, $\mathrm{H}$-atoms on heteroatoms were generally placed in the positions indicated by difference electron density maps and their positions were allowed to refine together with individual isotropic displacement parameters. All other $\mathrm{H}$-atoms were placed in geometrically calculated positions and refined by using a riding model, with $\mathrm{U}_{\mathrm{iso}}(\mathrm{H})=$ $1.2 \mathrm{U}_{\text {eq }}$ (parent atom) (1.5 $\mathrm{U}_{\text {eq }}$ for Me groups).

In the case of $\mathbf{6 a}$, the $\mathrm{Ph}$ ring of the middle benzyl group of the molecule is disordered over two orientations. The site occupation factor for the major conformation refined to 0.645(19). Similarity restraints were applied to the chemically equivalent bond lengths and angles involving all disordered $\mathrm{C}$-atoms, while neighbouring atoms within and between each

Centre via www.ccdc.cam.ac.uk/data_request/cif.

${ }^{10}$ ) We are grateful to $R$. Miller and C. Weeks of The Hauptman-Woodward Medical Research Institute, Buffalo, who carried out the solution of the structure using what was, at the time in 1996, a new, unreleased version of their program, SnB. Without their assistance, this structure determination would not nave been successful. 
conformation of the disordered ring were restrained to have similar atomic displacement parameters. There are disordered solvent molecules present in the crystal lattice, and these lie across centres of inversion, so that the ratio of peptide to solvent molecules is $2: 1$. The solvent appears to be a hexane molecule which has two orientations, although the end atoms in one orientation may be further disordered. As the disorder could not be modelled adequately, the contribution of the solvent molecules to the intensity data was removed by using the SQUEEZE routine [48] of the PLATON program [49]. Omission of the solvent molecules leaves two cavities of $217 \AA^{3}$ per unit cell. The number of electrons contributing to each void in the structure was calculated by the SQUEEZE routine to be approximately $41 \mathrm{e}$, although this may be an underbound. Allowing for one hexane molecule per cavity yields 50 e and this assumption was used in the subsequent calculation of the empirical formula, formula weight, density, linear absorption coefficient and $\mathrm{F}(000)$.

In the case of $(S, S)$-8e, the terminal benzyl group is disordered over two orientations with a site occupation factor for the major conformation of 0.669(5). Similarity restraints were applied to the chemically equivalent bond lengths and angles involving all disordered Catoms, while neighbouring atoms within and between each conformation of the disordered group were restrained to have similar atomic displacement parameters. The terminal methyl ester group might also be slightly disordered, because the atomic displacement parameters of these atoms, particularly $\mathrm{O}(24)$, are large. However the disorder could not be resolved reasonably and average positions were used for the atoms of this region of the molecule. As a result the $C(24)=O(24)$ bond appears to be somewhat shorter than it should be. The asymmetric unit also contains two sites which are partially occupied by water molecules. Initial refinement of the site occupation factors of these molecules gave values close to 0.5 and in the final refinement the site occupation factors of the water molecules were set to 0.5 . $\mathrm{H}$-atoms were not included for the water molecules. 
The crystals of $\mathbf{8 f}$ rapidly lost included solvent on standing in air. The asymmetric unit contains one peptide molecule plus approximately one third of a highly disordered hexane molecule which sits across a centre of inversion. As the disorder could not be modelled adequately, the contribution of the solvent molecules to the intensity data was removed by using the SQUEEZE routine of the PLATON program. Omission of the solvent molecules leaves four cavities of $128 \AA^{3}$ per unit cell. The number of electrons contributing to each void in the structure was calculated by the SQUEEZE routine to be approximately $31 \mathrm{e}$. Allowing for two thirds of a hexane molecule per cavity yields 30 e, which leads to a peptide:hexane ratio in the structure of 3:1 and this assumption was used in the subsequent calculations. The amide $\mathrm{H}$-atoms were placed in geometrically calculated positions and refined as described above for riding $\mathrm{H}$-atoms.

In the case of $(R, S, S)-\mathbf{8 g}$, the asymmetric unit contains one peptide molecule, one AcOEt molecule, and one partially occupied site for a $\mathrm{H}_{2} \mathrm{O}$ molecule. The site occupation factor for the $\mathrm{O}$-atom of $\mathrm{H}_{2} \mathrm{O}$ was initially refined and then fixed at 0.5 . The $\mathrm{H}$-atoms of the $\mathrm{H}_{2} \mathrm{O}$ molecule were included in the positions obtained from a difference electron density map and then constrained to ride on the parent $\mathrm{O}$-atom with $\mathrm{U}_{\text {iso }}(\mathrm{H})=1.5 \mathrm{U}_{\mathrm{eq}}(\mathrm{O})$.

The asymmetric unit of $(R, S)-\mathbf{8 h}$ contains two peptide, one hexane, and one half of a $\mathrm{H}_{2} \mathrm{O}$ molecule, where the latter sits on a two-fold axis. One of the peptide molecules has a disordered $\mathrm{Ph}$ ring in the $\mathrm{Z}$ group. Two sets of positions were defined for the atoms of this $\mathrm{Ph}$ ring and the site occupation factor of the major conformation of the ring refined to $0.504(1)$. Similarity restraints were applied to the chemically equivalent bond lengths and angles involving all disordered $\mathrm{C}$-atoms, the $\mathrm{C}-\mathrm{C}$ bonds were restrained to $1.395(5) \AA$, the two conformations of the ring were restrained to be planar, and neighbouring atoms within and between each conformation of the disordered $\mathrm{Ph}$ ring were restrained to have similar atomic displacement parameters. The amide $\mathrm{H}$-atoms were placed in geometrically calculated 
positions and refined as described above for riding $\mathrm{H}$-atoms. The position of the symmetryunique water $\mathrm{H}$-atom was refined with $\mathrm{U}_{\text {iso }}(\mathrm{H})=1.5 \mathrm{U}_{\mathrm{eq}}(\mathrm{O})$.

In the case of 10, the asymmetric unit contains one molecule of the peptide plus a disordered molecule of $\mathrm{MeOH}$. The site occupation factor of the major orientation of the $\mathrm{MeOH}$ molecule refined to $0.659(14)$. Neighbouring atoms within and between each conformation of the disordered $\mathrm{MeOH}$ molecule were restrained to have similar atomic displacement parameters. The amide and peptide hydroxy $\mathrm{H}$-atoms were placed in geometrically calculated positions and refined as described above for riding $\mathrm{H}$-atoms; for the hydroxy $\mathrm{H}$-atom $\mathrm{U}_{\text {iso }}(\mathrm{H})=1.5 \mathrm{U}_{\mathrm{eq}}(\mathrm{O})$. The hydroxy $\mathrm{H}$-atoms of the $\mathrm{MeOH}$ molecules were not included in the model.

The refinement of each structure was carried out on $F^{2}$ by using full-matrix leastsquares procedures, which minimized the function $\Sigma w\left(F_{\mathrm{o}}{ }^{2}-{F_{\mathrm{c}}}^{2}\right)^{2}$. A correction for secondary extinction was applied in the case of $\mathbf{6 a}$ and $(R, S)-\mathbf{8 h}$. Neutral atom scattering factors for non$\mathrm{H}$-atoms were taken from [50], and the scattering factors for $\mathrm{H}$-atoms were taken from [51]. Anomalous dispersion effects were included in $F_{\mathrm{c}}$ [52]; the values for $f$ and $f$ ' were those of [53]. The values of the mass attenuation coefficients are those of [54]. All refinements were performed using SHELXL-2013 [55].

Table 4. Crystallographic Data for Compounds 6a, 8a, $(S, S)-\mathbf{8 e}, \mathbf{8 f},(R, S, S)-\mathbf{8 g},(R, S)-$ 8h, and $\mathbf{1 0}$ 


\section{REFERENCES}

[1] D. Obrecht, H. Heimgartner, Helv. Chim. Acta 1981, 64, 482; D. Obrecht, H. Heimgartner, Helv. Chim. Acta 1987, 70, 102.

[2] a) P. Wipf, H. Heimgartner, Helv. Chim. Acta 1986, 69, 1153; b) P. Wipf, H. Heimgartner, Helv. Chim. Acta 1987, 70, 354; c) P. Wipf, H. Heimgartner, Helv. Chim. Acta 1988, 71, 140; d) P. Wipf, H. Heimgartner, Helv. Chim. Acta 1990, 73, 13.

[3] M. Sahebi, P. Wipf, H. Heimgartner, Tetrahedron 1989, 45, 2999.

[4] a) C. B. Bucher, A. Linden, H. Heimgartner, Helv. Chim. Acta 1995, 78, 935; b) C. B. Bucher, H. Heimgartner, Helv. Chim. Acta 1996, 79, 1903.

[5] C. Strässler, A. Linden, H. Heimgartner, Helv. Chim. Acta 1997, 80, 1528.

[6] K. A. Brun, A. Linden, H. Heimgartner, Helv. Chim. Acta 2008, 91, 526.

[7] I. Dannecker-Dörig, A. Linden, H. Heimgartner, Helv. Chim. Acta 2011, 94, 993.

[8] S. A. Stoykova, A. Linden, H. Heimgartner, Helv. Chim. Acta 2012, 95, 1325; S. A. Stoykova, A. Linden, H. Heimgartner, Helv. Chim. Acta 2013, 96, 1714.

[9] R. Luykx, C. B. Bucher, A. Linden, H. Heimgartner, Helv. Chim. Acta 1996, 79, 527; R. T. N. Luykx, A. Linden, H. Heimgartner, Helv. Chim. Acta 2003, 86, 4093.

[10] N. Pradeille, H. Heimgartner, J. Pept. Sci. 2003, 9, 827; N. Pradeille, O. Zerbe, K. Möhle, A. Linden, H. Heimgartner, Chem. Biodivers. 2005, 2, 1127; c) N. Pradeille, M. Tzouros, K. Möhle, A. Linden, H. Heimgartner, Chem. Biodivers. 2012, 9, 2528.

[11] W. Altherr, A. Linden, H. Heimgartner, Chem. Biodivers. 2007, 4, 1144; P. Blaser, W. Altherr, A. Linden, H. Heimgartner, Chem. Biodivers. 2013, 10, 920.

[12] a) S. Stamm, H. Heimgartner, Eur. J. Org. Chem. 2004, 3820; b) S. Stamm, A. Linden, H. Heimgartner, Helv. Chim. Acta 2006, 89, 1; c) S. Stamm, H. Heimgartner, Tetrahedron 2006, 62, 9671. 
[13] J. Lehmann, A. Linden, H. Heimgartner, Tetrahedron 1998, 54, 8721; J. Lehmann, A. Linden, H. Heimgartner, Tetrahedron 1999, 55, 5359; J. Lehmann, H. Heimgartner, Helv. Chim. Acta 1999, 82, 1899.

[14] R. A. Breitenmoser, H. Heimgartner, Helv. Chim. Acta 2001, 84, 786.

[15] A. Budzowski, A. Linden, H. Heimgartner, Helv. Chim. Acta 2008, 91, 1471.

[16] A. Bärtsch, B. Bischof, H. Heimgartner, Pol. J. Chem. 2009, 83, 195.

[17] K. A. Brun, A. Linden, H. Heimgartner, Helv. Chim. Acta 2001, 84, 1756.

[18] G. N. Ramachandran, C. Ramakrishnan, V. Sasisekharan, J. Mol. Biol. 1963, 7, 95; G. N. Ramachandran, V. Sasisekharan, Adv. Protein Chem. 1968, 23, 283.

[19] B. V. V. Prasad, P. Balaram, Crit. Rev. Biochem. Mol. Biol. 1984, 16, 307.

[20] C. Toniolo, E. Benedetti, ISI Atlas Sci.: Biochem. 1988, 5, 1238; C. Toniolo, E. Benedetti, Macromolecules 1991, 24, 4004; C. Toniolo, Biopolymers 1989, 28, 247.

[21] I. L. Karle, P. Balaram, Biochemistry 1990, 29, 6747.

[22] E. Benedetti, M. Saviano, R. Iacovino, C. Pedone, A. Santini, M. Crisma, F. Formaggio, C. Toniolo, Q. B. Broxterman, J. Kamphuis, Biopolymers 1998, 46, 433.

[23] S. Aravinda, N. Shamala, P. Balaram, Chem. Biodiversity 2008, 5, 1238; U. S. Raghavender, S. Aravinda, N. Shamala, Katharaju, R. K. Rai, P. Balaram, J. Am. Chem. Soc. 2009, 131, 15130; U. S. Raghavender, Katharaju, S. Aravinda, N. Shamala, P. Balaram, J. Am. Chem. Soc. 2010, 132, 1075; B. Dinesh, K. Basuroy, N. Shamala, P. Balaram, Tetrahedron 2012, 68, 4374; K. Basuroy, V. Karuppiah, N. Shamala, P. Balaram, Helv. Chim. Acta 2012, 95, 2589.

[24] T. Yamada, S. Fujioka, M. Takeda, S. Gohda, T. Murashima, Y. In, Pept. Sci. (2010), 47 ${ }^{\text {th }}$, 136; Y. In, K. Minoura, T. Ishida, S. Fujioka, M. Takeda, T. Murashima, T. Yamada, Pept. Sci. (2011), $48^{\text {th }}, 205$.

[25] a) T. Jeremic, A. Linden, H. Heimgartner, Chem. Biodivers. 2004, 1, 1730; b) T. Jeremic, A. Linden, H. Heimgartner, Helv. Chim. Acta 2004, 87, 3056. 
[26] a) K. Dietliker, H. Heimgartner, Helv. Chim. Acta 1983, 66, 262; b) J. M. Villalgordo, H. Heimgartner, Helv. Chim. Acta 1995, 78, 1983.

[27] P. Wipf, Ph.D. thesis, Universität Zürich, 1987.

[28] H.-D. Jakubke, C. Klessen, E. Berger, K. Neubert, Tetrahedron Lett. 1978, 1497.

[29] S. Chen, J. Xu, Tetrahedron Lett. 1992, 33, 647.

[30] J. V. Hruby, A. I. Brewster, J. A. Glasel, Proc. Natl. Acad. Sci. 1971, 68, 450.

[31] a) C. Grathwohl, K. Wüthrich, Biopolymers 1976, 15, 2025; b) S. K. Sarkar, P. E. Young, C. E. Sullivan, D. A. Torchia, Proc. Natl. Acad. Sci. 1984, 81, 4800; c) H. Kessler, Angew. Chem. Int. Ed. Engl. 1982, 21, 512.

[32] C. K. Johnson, ORTEP II, Report ORNL-5138, Oak Ridge National Laboratory, Oak Ridge, Tennessee, 1976.

[33] C. M. Venkatachalam, Biopolymers 1968, 6, 1425; P. N. Lewis, F. A. Momany, H. A. Scheraga, Biochem. Biophys. Acta 1973, 303, 211.

[34] J. Bernstein, R. E. Davis, L. Shimoni, N.-L. Chang, Angew. Chem. Int. Ed. 1995, 34, 1555.

[35] C. Toniolo, G. M. Bonora, M. Crisma, E. Benedetti, A. Bavoso, B. Di Blasio, V. Pavone, C. Pedone, Int. J. Pept. Protein Res. 1983, 22, 603; E. G. Hutchinson, J. M. Thornton, Protein Sci. 1994, 3, 2207.

[36] a) F. Formaggio, S. Pegoraro, M. Crisma, G. Valle, C. Toniolo, G. Précigoux, W. H. J. Boesten, H. E. Schoemaker, J. Kamphuis, J. Biomol. Struct. Dyn. 1993, 10, 919; b) E. Benedetti, Biopolymers 1996, 40, 3.

[37] I. Dannecker-Dörig, A. Linden, H. Heimgartner, Coll. Czech. Chem. Commun. 2009, 74, 901.

[38] L. A. Carpino, A. A. Abdel-Maksoud, E. M. E. Mansour, M. A. Zewail, Tetrahedron Lett. 2007, 48, 7407.

[39] a) G. R. Marshall, E. E. Hodgkin, D. A. Langs, G. D. Smith, J. Zabrocki, M. T. Leplawy, Proc. Natl. Acad. Sci. 1990, 87, 487; b) C. Toniolo, M. Crisma, F. Formaggio, G. Valle, G. 
Cavicchioni, G. Précigoux, Biopolymers 1993, 33, 167; c) B. Di Blasio, V. Pavone, A. Lombardi, C. Pedone, E. Benedetti, Biopolymers 1993, 33, 1037; d) R. Banerjee, S. Chattopadhyay, G. Basu, Proteins 2009, 76, 184.

[40] a) C. Toniolo, G. M. Bonora, A. Bavoso, E. Benedetti, B. Di Blasio, V. Pavone, C. Pedone, Biopolymers 1983, 22, 205; b) B. Pengo, F. Formaggio, M. Crisma, C. Toniolo, G. M. Bonora, Q. B. Broxterman, J. Kamphuis, M. Saviano, R. Iacovino, F. Rossi, E. Benedetti, J. Chem. Soc., Perkin Trans. 2 1998, 1651.

[41] C. Toniolo, F. Formaggio, M. Crisma, G. Valle, W. H. J. Boesten, H. E. Schoemaker, J. Kamphuis, P. A. Temussi, E. L. Becker, G. Précigoux, Tetrahedron 1993, 49, 3641.

[42] M. De Poli, M. De Zotti, J. Raftery, J. A. Aguilar, G. A. Morris, J. Clayden, J. Org. Chem. 2013, 78, 2248.

[43] Y. Demizu, M. Tanaka, M. Nagano, M. Kurihara, M. Doi, T. Maruyama, H. Suemune, Chem. Pharm. Bull. 2007, 55, 840; Y. Demizu, Y. U. Yabuki, M. Doi, Y. Sato, M. Tanaka, M. Kurihara, J. Pept. Sci. 2012, 18, 466.

[44] S. Ram, L. D. Spicer, Tetrahedron Lett. 1987, 28, 515.

[45] A. C. T. North, CD. C. Phillips, F. S. Mathews, Acta Crystallogr., Sect. A 1968, 24, 351.

[46] G. M. Sheldrick, Acta Crystallogr., Sect. A 1990, 46, 467.

[47] R. Miller, S. M. Gallo, H. G. Khalak, C. M. Weeks, J. Appl. Crystallogr. 1994, 27, 613.

[48] P. van der Sluis, A. L. Spek, Acta Crystallogr. Sect. A, 1990, 46, 194

[49] A. L. Spek, Acta Crystallogr. Sect. D, 2009, 65, 148-155.

[50] E. N. Maslen, A. G. Fox, M. A. O'Keefe, 'International Tables for Crystallography', Ed. A. J. C. Wilson, Kluwer Academic Publishers, Dordrecht, 1992, Vol. C, Table 6.1.1.1, p. 477.

[51] R. F. Stewart, E. R. Davidson, W. T. Simpson, J. Chem. Phys. 1965, 42, 3175.

[52] J. A. Ibers, W. C. Hamilton, Acta Crystallogr. 1964, 17, 781.

[53] D. C. Creagh, W. J. McAuley, 'International Tables for Crystallography', Ed. A. J. C. Wilson, Kluwer Academic Publishers, Dordrecht, 1992, Vol. C, Table 4.2.6.8, p. 219. 
[54] D. C. Creagh, J. H. Hubbell, 'International Tables for Crystallography', Ed. A. J. C. Wilson, Kluwer Academic Publishers, Dordrecht, 1992, Vol. C, Table 4.2.4.3, p. 200.

[55] G. M. Sheldrick, Acta Crystallogr., Sect. A 2008, 64, $112 .$. 
Legends

Fig. 1. ORTEP Plot [32] of the molecular structure of the tetrapeptide 6a (50\% probability ellipsoids, arbitrary numbering of atoms, H-atoms bonded to C-atoms omitted for clarity and only the major conformation of the disordered central benzyl ring is shown, solvent molecule not included)

Fig. 2. ORTEP Plot [32] of the molecular structure of the pentapeptide 8a (50\% probability ellipsoids, arbitrary numbering of atoms, $\mathrm{H}$-atoms bonded to $\mathrm{C}$-atoms omitted for clarity)

Fig. 3. ORTEP Plot [32] of the molecular structure of the pentapeptide $\mathbf{8 f}$ (50\% probability ellipsoids, arbitrary numbering of atoms, $\mathrm{H}$-atoms bonded to C-atoms omitted for clarity, solvent molecule not included)

Fig. 4. ORTEP Plot [32] of the molecular structure of the pentapeptide $(S, S)-8 \mathbf{e}(50 \%$ probability ellipsoids, arbitrary numbering of atoms, $\mathrm{H}$-atoms bonded to $\mathrm{C}$-atoms omitted for clarity and only the major conformation of the disordered terminal benzyl group is shown, water molecules not included)

Fig. 5. ORTEP Plot [32] of the molecular structure of the pentapeptide $(R, S, S)-\mathbf{8 g}(50 \%$ probability ellipsoids, arbitrary numbering of atoms, $\mathrm{H}$-atoms bonded to $\mathrm{C}$-atoms omitted for clarity, solvent molecules not included)

Fig. 6. ORTEP Plot [32] of the molecular structure of one of the two symmetryindependent molecules of the pentapeptide $(R, S)-\mathbf{8 h}(50 \%$ probability ellipsoids, arbitrary 
numbering of atoms, $\mathrm{H}$-atoms bonded to $\mathrm{C}$-atoms omitted for clarity, solvent molecules not included)

Fig. 7. ORTEP Plot [32] of the molecular structure of the pentapeptide 10 (50\% probability ellipsoids, arbitrary numbering of atoms, H-atoms bonded to C-atoms omitted for clarity, solvent molecule not included)

Table 1. ${ }^{13} C$-NMR Chemical Shifts of $C(\beta)$ and $C(\gamma)$ of the Pro Residue in Pro-Containing Pentapeptides

Table 2. Torsion Angles $\omega, \phi$, and $\psi$ of the Backbone of the Pentapeptides $\mathbf{6 a}, \mathbf{8 a},(S, S)-\mathbf{8 e}$, 8f, $(R, S, S)-\mathbf{8 g},(R, S)-\mathbf{8 h}$, and $\mathbf{1 0}$ in the Crystal (Amino Acid $(i)=\mathrm{Gly}(1))$

Table 3. Intramolecular H-Bonds of Compounds $\mathbf{8 a},(S, S)-\mathbf{8 e}, \mathbf{8 f},(R, S, S)-\mathbf{8 g},(R, S)-\mathbf{8 h}$, and 10 (atom numbering refers to Figs. $1-7$ )

Table 4. Crystallographic Data for Compounds $\mathbf{6 a}, \mathbf{8 a},(S, S)-\mathbf{8 e}, \mathbf{8 f},(R, S, S)-\mathbf{8 g},(R, S)-\mathbf{8 h}$, and $\mathbf{1 0}$ 
Table $1 .{ }^{13} C$-NMR Chemical Shifts of $C(\beta)$ and $C(\gamma)$ of the Pro Residue in Pro-Containing Pentapeptides

\begin{tabular}{|l|l|l|l|}
\hline Peptide & Solvent & $\mathrm{C}(\beta)[\mathrm{ppm}]$ & $\mathrm{C}(\gamma)[\mathrm{ppm}]$ \\
\hline & & & \\
Z-Gly-Aib-Pro-Aib-Aib-N(Me)Ph (8d) & $\mathrm{CDCl}_{3}$ & 28.8 & 26.2 \\
Z-Gly-( $R$ )-Phe(2Me)-Pro-Aib-Phe-OMe $((R, S, S)$-8g $)$ & $\mathrm{CDCl}_{3}$ & 28.5 & 26.0 \\
Z-Gly-(S)-Phe(2Me)-Pro-Aib-Phe-OMe $((S, S, S)$-8g) & $\mathrm{CDCl}_{3}$ & 28.3 & 26.0 \\
Z-Gly-(R)-Phe(2Me)-Pro-Aib-Aib-N(Me)Ph $((R, S)-8 \mathbf{8 h})$ & $\mathrm{CDCl}_{3}$ & 28.7 & 26.3 \\
Z-Gly-(S)-Phe(2Me)-Pro-Aib-Aib-N(Me)Ph $((S, S)-\mathbf{8 h})$ & $\mathrm{CDCl}_{3}$ & 28.6 & 26.2 \\
Z-Gly-Aib-Pro-Aib-Aib-OH (10) & $\left(\mathrm{D}_{6}\right)$ DMSO & 28.2 & 25.5 \\
& & & \\
\hline
\end{tabular}


Table 2. Torsion Angles $\phi, \psi$, and $\omega$ of the Backbone of Compounds $\mathbf{8 a},(S, S)-\mathbf{8 e}, \mathbf{8 f}$, $(R, S, S)-\mathbf{8 g},(R, S)-\mathbf{8 h}$, and $\mathbf{1 0}$ in the Crystal (Amino Acid $(i)=\mathrm{Gly}(1))$

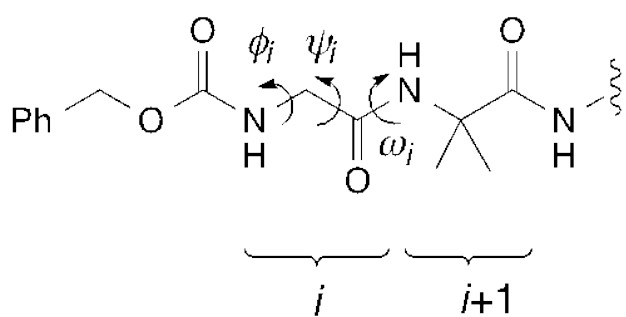

\begin{tabular}{|l|l|l|l|l|l|l|}
\hline & $\mathbf{8}$ & $(S, S)-\mathbf{8}$ & $\mathbf{8 f}$ & $(R, S, S)-\mathbf{8 g}$ & $R, S)-\mathbf{8 h}$ & $\mathbf{1 0}$ \\
\hline$\phi_{(i)}$ & $53.7(2)$ & $-96.3(3)$ & $-88.7(5)$ & $70.6(5)$ & $64(1) ; 62(1)$ & $82.2(6)$ \\
$\psi_{(i)}$ & $32.4(2)$ & $-5.6(5)$ & $-33.9(6)$ & $179.0(4)$ & $-170.3(7) ;-172.3(7)$ & $155.3(5)$ \\
$\omega_{(i)}$ & $-178.7(1)$ & $173.2(3)$ & $178.7(4)$ & $179.4(4)$ & $175.1(7) ; 177.9(7)$ & $-169.2(4)$ \\
\hline$\phi_{(i+1)}$ & $55.8(2)$ & $53.9(4)$ & $49.4(6)$ & $-49.6(5)$ & $-51.5(9) ;-51.0(9)$ & $-53.8(6)$ \\
$\psi_{(i+1)}$ & $21.8(2)$ & $34.3(5)$ & $42.2(6)$ & $-43.4(5)$ & $-36(1) ;-40.5(9)$ & $-37.8(6)$ \\
$\omega_{(i+1)}$ & $-178.7(1)$ & $179.5(3)$ & $173.1(4)$ & $-173.7(4)$ & $-178.9(7) ;-177.8(6)$ & $-176.9(4)$ \\
\hline$\phi_{(i+2)}$ & $51.9(2)$ & $61.3(5)$ & $61.3(6)$ & $-66.2(5)$ & $-55(1) ;-57(1)$ & $-61.2(6)$ \\
$\psi_{(i+2)}$ & $29.6(2)$ & $13.5(5)$ & $29.4(6)$ & $-13.6(6)$ & $-30(1) ;-30(1)$ & $-31.7(6)$ \\
$\omega_{(i+2)}$ & $178.6(1)$ & $-170.8(3)$ & $-177.5(4)$ & $171.5(4)$ & $-179.0(7) ;-178.1(7)$ & $178.7(4)$ \\
\hline$\phi_{(i+3)}$ & $57.0(2)$ & $55.8(4)$ & $59.4(6)$ & $-55.6(5)$ & $-64.8(9) ;-58.4(9)$ & $-54.1(7)$ \\
$\psi_{(i+3)}$ & $30.4(2)$ & $30.6(5)$ & $32.4(6)$ & $-32.0(5)$ & $-30(1) ;-32.6(9)$ & $-39.5(5)$ \\
$\omega_{(i+3)}$ & $166.3(1)$ & $163.6(4)$ & $-170.7(4)$ & $-172.5(4)$ & $-162.1(8) ;-154.6(7)$ & $175.4(4)$ \\
\hline$\phi_{(i+4)}$ & $-83.0(2)$ & $-81.0(5)$ & $-47.4(7)$ & $-111.0(5)$ & $-57(1) ;-52(1)$ & $49.4(6)$ \\
$\psi_{(i+4)}$ & $162.8(1)$ & $-179.1(4)$ & $-57.4(7)$ & $179.4(4)$ & $-50(1) ;-54(1)$ & $45.2(6)$ \\
\hline
\end{tabular}


Table 3. Intramolecular H-Bonds of Compounds $\mathbf{8 a},(S, S)-\mathbf{8 e}, \mathbf{8 f},(R, S, S)-\mathbf{8 g},(R, S)-\mathbf{8 h}$, and 10 (atom numbering refers to Figs. $1-7$ )

\begin{tabular}{|c|c|c|c|c|c|c|}
\hline \multirow[t]{2}{*}{ Compound } & \multicolumn{2}{|c|}{$\mathrm{N}(1)-\mathrm{H}^{\cdots \cdots} \mathrm{O}(8)$} & \multicolumn{2}{|c|}{$\mathrm{N}(4)-\mathrm{H}^{\cdots \cdots} \mathrm{O}(11)$} & \multicolumn{2}{|c|}{$\mathrm{N}(7)-\mathrm{H}^{\cdots \cdots} \mathrm{O}(14)$} \\
\hline & $\mathrm{N}^{\cdots \cdots} \mathrm{O}(\AA)$ & $\mathrm{N}-\mathrm{H}^{\cdots \cdots} \mathrm{O}\left({ }^{\circ}\right)$ & $\mathrm{N}^{\cdots \cdots} \mathrm{O}(\AA)$ & $\mathrm{N}-\mathrm{H}^{\cdots \cdots} \mathrm{O}(\AA)$ & $\mathrm{N}^{\cdots \cdots} \mathrm{O}(\AA)$ & $\mathrm{N}-\mathrm{H}^{\cdots \cdots} \mathrm{O}(\AA)$ \\
\hline $8 a$ & $2.928(2)$ & $163(2)$ & $3.052(2)$ & $171(2)$ & $3.013(2)$ & $166(1)$ \\
\hline$(\mathrm{S}, \mathrm{S})-\mathbf{8 e}$ & $3.007(4)$ & $165(4)$ & $2.872(4)$ & $166(4)$ & - & \\
\hline $8 f$ & $2.976(5)$ & 130 & $2.977(5)$ & 141 & - & \\
\hline$(R, S, S)-\mathbf{8 g}$ & $2.960(5)$ & $162(4)$ & $3.001(5)$ & $161(4)$ & - & \\
\hline \multirow[t]{2}{*}{$(R, S)-\mathbf{8 h}$} & $2.974(8)$ & 163 & $3.036(8)$ & 149 & - & \\
\hline & $3.055(7)$ & 168 & $3.067(8)$ & 147 & & \\
\hline 10 & $3.034(5)$ & 126 & $3.000(5)$ & 140 & - & \\
\hline
\end{tabular}


Table 4. Crystallographic Data for Compounds $\mathbf{6 a}, \mathbf{8 a},(S, S)-\mathbf{8 e}, \mathbf{8 f},(R, S, S)-\mathbf{8 g},(R, S)-\mathbf{8 h}$, and 10

\begin{tabular}{|c|c|c|c|c|}
\hline & & $\mathbf{6 a}$ & $\mathbf{8 a}$ & $(S, S)-\mathbf{8 e}$ \\
\hline \multicolumn{2}{|l|}{ Crystallized from } & $\mathrm{CH}_{2} \mathrm{Cl}_{2} / \mathrm{Et}_{2} \mathrm{O} /$ hexane & $\mathrm{CH}_{2} \mathrm{Cl}_{2} /$ hexane & AcOEt/hexane \\
\hline \multicolumn{2}{|l|}{ Empirical formula } & $\mathrm{C}_{35} \mathrm{H}_{43} \mathrm{~N}_{5} \mathrm{O}_{6} \cdot 0.5 \mathrm{C}_{6} \mathrm{H}_{14}$ & $\mathrm{C}_{31} \mathrm{H}_{41} \mathrm{~N}_{5} \mathrm{O}_{8}$ & $\mathrm{C}_{36} \mathrm{H}_{43} \mathrm{~N}_{5} \mathrm{O}_{8} \cdot \mathrm{H}_{2} \mathrm{O}$ \\
\hline \multicolumn{2}{|l|}{ Formula weight } & 672.84 & 611.69 & 691.78 \\
\hline \multicolumn{2}{|l|}{ Crystal color, habit } & colorless, prism & colorless, prism & colorless, irregular prism \\
\hline \multicolumn{2}{|c|}{ Crystal dimensions [mm] } & $0.23 \times 0.25 \times 0.45$ & $0.33 \times 0.41 \times 0.43$ & $0.25 \times 0.27 \times 0.50$ \\
\hline \multicolumn{2}{|l|}{ Temperature $[\mathrm{K}]$} & 173(1) & 173(1) & $173(1)$ \\
\hline \multicolumn{2}{|l|}{ Crystal system } & monoclinic & monoclinic & orthorhombic \\
\hline \multicolumn{2}{|l|}{ Space group } & $P 2_{1} / n$ & $P 2_{1} / n$ & $P 2{ }_{1}{ }_{1} 2_{1}$ \\
\hline \multicolumn{2}{|l|}{$Z$} & 4 & 4 & 4 \\
\hline \multicolumn{2}{|c|}{ Reflections for cell determination } & 22 & 25 & 25 \\
\hline \multicolumn{2}{|c|}{$2 \theta$ range for cell determination $\left[{ }^{\circ}\right]$} & $32-39$ & $38-40$ & $23-33$ \\
\hline \multirow[t]{7}{*}{ Unit cell parameters } & $a[\AA]$ & $11.642(3)$ & $13.698(3)$ & $15.347(4)$ \\
\hline & $b[\AA]$ & $15.135(3)$ & $13.979(4)$ & $24.232(4)$ \\
\hline & $c[\AA]$ & $21.307(2)$ & $17.409(3)$ & $10.028(4)$ \\
\hline & $\alpha\left[^{\circ}\right]$ & 90 & 90 & 90 \\
\hline & $\beta\left[^{\circ}\right]$ & $97.294(12)$ & $109.133(14)$ & 90 \\
\hline & $\gamma\left[^{\circ}\right]$ & 90 & 90 & 90 \\
\hline & $V\left[\AA^{3}\right]$ & $3724(1)$ & $3150(1)$ & $3729(2)$ \\
\hline \multicolumn{2}{|l|}{$D_{x}\left[\mathrm{~g} \mathrm{~cm}^{-3}\right]$} & 1.200 & 1.290 & 1.232 \\
\hline \multicolumn{2}{|l|}{$\mu\left(\operatorname{Mo} K_{\alpha}\right)\left[\mathrm{mm}^{-1}\right]$} & 0.817 & 0.0940 & 0.0892 \\
\hline \multicolumn{2}{|l|}{ Scan type } & $\omega / 2 \theta$ & $\omega / 2 \theta$ & $\omega$ \\
\hline \multicolumn{2}{|l|}{$2 \theta_{(\max )}\left[^{\circ}\right]$} & 55 & 60 & 55 \\
\hline \multicolumn{2}{|c|}{ Transmission factors (min; max) } & - & - & - \\
\hline \multicolumn{2}{|c|}{ Total reflections measured } & 9296 & 9898 & 5535 \\
\hline \multicolumn{2}{|c|}{ Symmetry independent reflections } & 8539 & 9182 & 5389 \\
\hline \multicolumn{2}{|c|}{ Reflections used $[I>2 \sigma(I)]$} & 4362 & 6222 & 3892 \\
\hline \multicolumn{2}{|c|}{ Parameters refined; restraints } & $484 ; 164$ & $423 ; 0$ & $548 ; 202$ \\
\hline \multirow{2}{*}{\multicolumn{2}{|c|}{$\begin{array}{ll}\text { Final } & R(F)[I>2 \sigma(I) \text { reflections }] \\
& w R\left(F^{2}\right) \text { (all data) }\end{array}$}} & 0.0577 & 0.0454 & 0.0549 \\
\hline & & 0.1671 & 0.1262 & 0.1682 \\
\hline \multicolumn{2}{|c|}{ Weighting parameter $\left.(a ; b)^{\mathrm{a}}\right)$} & $0.0830 ; 0$ & $0.0525 ; 0.4262$ & $0.0886 ; 0.8267$ \\
\hline \multicolumn{2}{|c|}{ Secondary extinction coefficient } & $0.0022(6)$ & - & - \\
\hline \multicolumn{2}{|l|}{ Goodness of fit } & 0.927 & 1.034 & 1.058 \\
\hline \multicolumn{2}{|l|}{ Final $\Delta_{\max } / \sigma$} & 0.000 & 0.000 & 0.000 \\
\hline \multicolumn{2}{|l|}{$\Delta \rho(\max ; \min )\left[\mathrm{e} \AA^{-3}\right]$} & $0.29 ;-0.24$ & $0.37 ;-0.18$ & $0.79 ;-0.26$ \\
\hline
\end{tabular}

$\left.{ }^{\mathrm{a}}\right) w^{-1}=\sigma^{2}\left(F_{\mathrm{o}}^{2}\right)+(a P)^{2}+b P$, where $P=\left(F_{\mathrm{o}}{ }^{2}+2 F_{\mathrm{c}}{ }^{2}\right) / 3$ 
Table 4. Crystallographic Data for Compounds $\mathbf{6 a}, \mathbf{8 a},(S, S)-\mathbf{8 e}, \mathbf{8 f},(R, S, S)-\mathbf{8 g},(R, S)-\mathbf{8 h}$, and 10 (continued)

\begin{tabular}{|c|c|c|c|c|}
\hline & & $8 f$ & $(R, S, S)-\mathbf{8 g}$ & $(R, S)-\mathbf{8 h}$ \\
\hline \multicolumn{2}{|l|}{ Crystallized from } & AcOEt/hexane & $\mathrm{MeOH} / \mathrm{AcOEt}$ & AcOEt/MeOH/hexane \\
\hline \multicolumn{2}{|l|}{ Empirical formula } & ${ }_{37} \mathrm{H}_{46} \mathrm{~N}_{6} \mathrm{O}_{7} \cdot 0.33 \mathrm{C}_{6} \mathrm{H}_{14} \quad \mathrm{C}_{39} \mathrm{H}$ & ${ }_{39} \mathrm{H}_{47} \mathrm{~N}_{5} \mathrm{O}_{8} \cdot \mathrm{C}_{4} \mathrm{H}_{8} \mathrm{O}_{2} \cdot 0.5 \mathrm{H}_{2} \mathrm{O}$ & $\mathrm{C}_{40} \mathrm{H}_{50} \mathrm{~N}_{6} \mathrm{O}_{7} 0.5 \mathrm{C}_{6} \mathrm{H}_{14} \cdot 0.25 \mathrm{H}_{2} \mathrm{C}$ \\
\hline \multicolumn{2}{|l|}{ Formula weight } & 715.33 & 810.94 & 774.46 \\
\hline \multicolumn{2}{|l|}{ Crystal color, habit } & colorless, plate & colorless, plate & colorless, hexagonal prism \\
\hline \multicolumn{2}{|c|}{ Crystal dimensions [mm] } & $0.10 \times 0.38 \times 0.42$ & $0.20 \times 0.45 \times 0.50$ & $0.36 \times 0.36 \times 0.38$ \\
\hline \multicolumn{2}{|l|}{ Temperature $[\mathrm{K}]$} & $173(1)$ & $173(1)$ & $173(1)$ \\
\hline \multicolumn{2}{|l|}{ Crystal system } & orthorhombic & orthorhombic & trigonal \\
\hline \multicolumn{2}{|l|}{ Space group } & Pbca & $P 2_{1} 2_{1} 2_{1}$ & $P 3_{1} 21$ \\
\hline \multicolumn{2}{|l|}{$Z$} & 8 & 4 & 12 \\
\hline \multicolumn{2}{|c|}{ Reflections for cell determination } & 25 & 25 & 25 \\
\hline \multicolumn{2}{|c|}{$2 \theta$ range for cell determination $\left[{ }^{\circ}\right]$} & $21-25$ & $24-35$ & $20-27$ \\
\hline \multirow[t]{7}{*}{ Unit cell parameters } & $a[\AA]$ & $25.465(4)$ & $16.601(5)$ & $15.706(6)$ \\
\hline & $b[\AA]$ & $20.729(6)$ & $26.785(8)$ & $15.706(6)$ \\
\hline & $c[\AA]$ & $14.536(5)$ & $9.720(6)$ & $60.971(6)$ \\
\hline & $\alpha\left[^{\circ}\right]$ & 90 & 90 & 90 \\
\hline & $\beta\left[^{\circ}\right]$ & 90 & 90 & 90 \\
\hline & $\gamma\left[^{\circ}\right]$ & 90 & 90 & 120 \\
\hline & $V\left[\AA^{3}\right]$ & $7673(4)$ & $4322(3)$ & $13026(11)$ \\
\hline \multicolumn{2}{|l|}{$D_{x}\left[\mathrm{~g} \mathrm{~cm}^{-3}\right]$} & 1.239 & 1.246 & 1.185 \\
\hline \multicolumn{2}{|l|}{$\mu\left(\mathrm{Mo} K_{\alpha}\right)\left[\mathrm{mm}^{-1}\right]$} & 0.0860 & 0.0895 & 0.0813 \\
\hline \multicolumn{2}{|l|}{ Scan type } & $\omega$ & $\omega$ & $\omega$ \\
\hline \multicolumn{2}{|l|}{$2 \theta_{(\max )}\left[^{\circ}\right]$} & 50 & 50 & 50 \\
\hline \multicolumn{2}{|c|}{ Transmission factors (min; max) } & $0.806 ; 1.000$ & $0.903 ; 1.000$ & - \\
\hline \multicolumn{2}{|c|}{ Total reflections measured } & 8431 & 5016 & 29374 \\
\hline \multicolumn{2}{|c|}{ Symmetry independent reflections } & 6768 & 4874 & 15416 \\
\hline \multicolumn{2}{|c|}{ Reflections used $[I>2 \sigma(I)]$} & 2204 & 3299 & 8853 \\
\hline \multicolumn{2}{|c|}{ Parameters refined; restraints } & $457 ; 0$ & $554 ; 0$ & $1087 ; 187$ \\
\hline Final $\quad R(F)[I>20$ & reflections] & 0.0701 & 0.0446 & 0.0787 \\
\hline$w R\left(F^{2}\right)($ all d & & 0.1842 & 0.1104 & 0.2097 \\
\hline \multicolumn{2}{|c|}{ Weighting parameter $\left.(a ; b)^{\mathrm{a}}\right)$} & $0.0781 ; 0$ & $0.0392 ; 0.9522$ & $0.0641 ; 16.507$ \\
\hline \multicolumn{2}{|c|}{ Secondary extinction coefficient } & - & - & $0.0014(2)$ \\
\hline \multicolumn{2}{|l|}{ Goodness of fit } & 0.790 & 1.013 & 1.057 \\
\hline \multicolumn{2}{|l|}{ Final $\Delta_{\max } / \sigma$} & 0.000 & 0.000 & 0.002 \\
\hline \multicolumn{2}{|l|}{$\Delta \rho(\max ; \min )\left[\mathrm{e} \AA^{-3}\right]$} & $0.32 ;-0.25$ & $0.19 ;-0.21$ & $0.42 ;-0.38$ \\
\hline
\end{tabular}


Table 4. Crystallographic Data for Compounds $\mathbf{6 a}, \mathbf{8 a},(S, S)-\mathbf{8 e}, \mathbf{8 f},(R, S, S)-\mathbf{8 g},(R, S)-\mathbf{8 h}$, and 10 (continued)

10

\begin{tabular}{|c|c|c|}
\hline \multicolumn{2}{|l|}{ Crystallized from } & $\mathrm{MeOH}$ \\
\hline \multicolumn{2}{|l|}{ Empirical formula } & $\mathrm{C}_{27} \mathrm{H}_{39} \mathrm{~N}_{5} \mathrm{O}_{8} \cdot \mathrm{CH}_{4} \mathrm{O}$ \\
\hline \multicolumn{2}{|l|}{ Formula weight } & 593.67 \\
\hline \multicolumn{2}{|l|}{ Crystal color, habit } & colorless, prism \\
\hline \multicolumn{2}{|c|}{ Crystal dimensions $[\mathrm{mm}]$} & $0.18 \times 0.33 \times 0.50$ \\
\hline \multicolumn{2}{|l|}{ Temperature $[\mathrm{K}]$} & $173(1)$ \\
\hline \multicolumn{2}{|l|}{ Crystal system } & orthorhombic \\
\hline \multicolumn{2}{|l|}{ Space group } & $P 2_{1} 2_{1} 2_{1}$ \\
\hline \multicolumn{2}{|l|}{$Z$} & 4 \\
\hline \multicolumn{2}{|c|}{ Reflections for cell determination } & 17 \\
\hline \multicolumn{2}{|c|}{$2 \theta$ range for cell determination $\left[{ }^{\circ}\right]$} & $20-26$ \\
\hline \multirow[t]{7}{*}{ Unit cell parameters } & $a[\AA]$ & $12.983(9)$ \\
\hline & $b[\AA]$ & $10.249(5)$ \\
\hline & $c[\AA]$ & $23.918(7)$ \\
\hline & $\alpha\left[^{\circ}\right]$ & 90 \\
\hline & $\beta\left[^{\circ}\right]$ & 90 \\
\hline & $\gamma\left[^{\circ}\right]$ & 90 \\
\hline & $V\left[\AA^{3}\right]$ & $3183(3)$ \\
\hline \multicolumn{2}{|l|}{$D_{x}\left[\mathrm{~g} \mathrm{~cm}^{-3}\right]$} & 1.239 \\
\hline \multicolumn{2}{|l|}{$\mu\left(\operatorname{Mo} K_{\alpha}\right)\left[\mathrm{mm}^{-1}\right]$} & 0.0928 \\
\hline \multicolumn{2}{|l|}{ Scan type } & $\omega / 2 \theta$ \\
\hline \multicolumn{2}{|l|}{$2 \theta_{(\max )}\left[^{\circ}\right]$} & 55 \\
\hline \multicolumn{2}{|c|}{ Transmission factors (min; max) } & - \\
\hline \multicolumn{2}{|c|}{ Total reflections measured } & 4118 \\
\hline \multicolumn{2}{|c|}{ Symmetry independent reflections } & 4090 \\
\hline \multicolumn{2}{|c|}{ Reflections used $[I>2 \sigma(I)]$} & 2798 \\
\hline \multicolumn{2}{|c|}{ Parameters refined; restraints } & $407 ; 36$ \\
\hline Final $R(F)[I>20$ & reflections] & 0.0611 \\
\hline$w R\left(F^{2}\right)($ all d & & 0.1975 \\
\hline \multicolumn{2}{|c|}{ Weighting parameter $\left.(a ; b)^{\mathrm{a}}\right)$} & $0.0808 ; 2.3471$ \\
\hline \multicolumn{2}{|c|}{ Secondary extinction coefficient } & - \\
\hline \multicolumn{2}{|l|}{ Goodness of fit } & 1.085 \\
\hline \multicolumn{2}{|l|}{ Final $\Delta_{\max } / \sigma$} & 0.000 \\
\hline \multicolumn{2}{|l|}{$\Delta \rho(\max ; \min )\left[\mathrm{e} \AA^{-3}\right]$} & $0.33 ;-0.36$ \\
\hline
\end{tabular}


Scheme 1
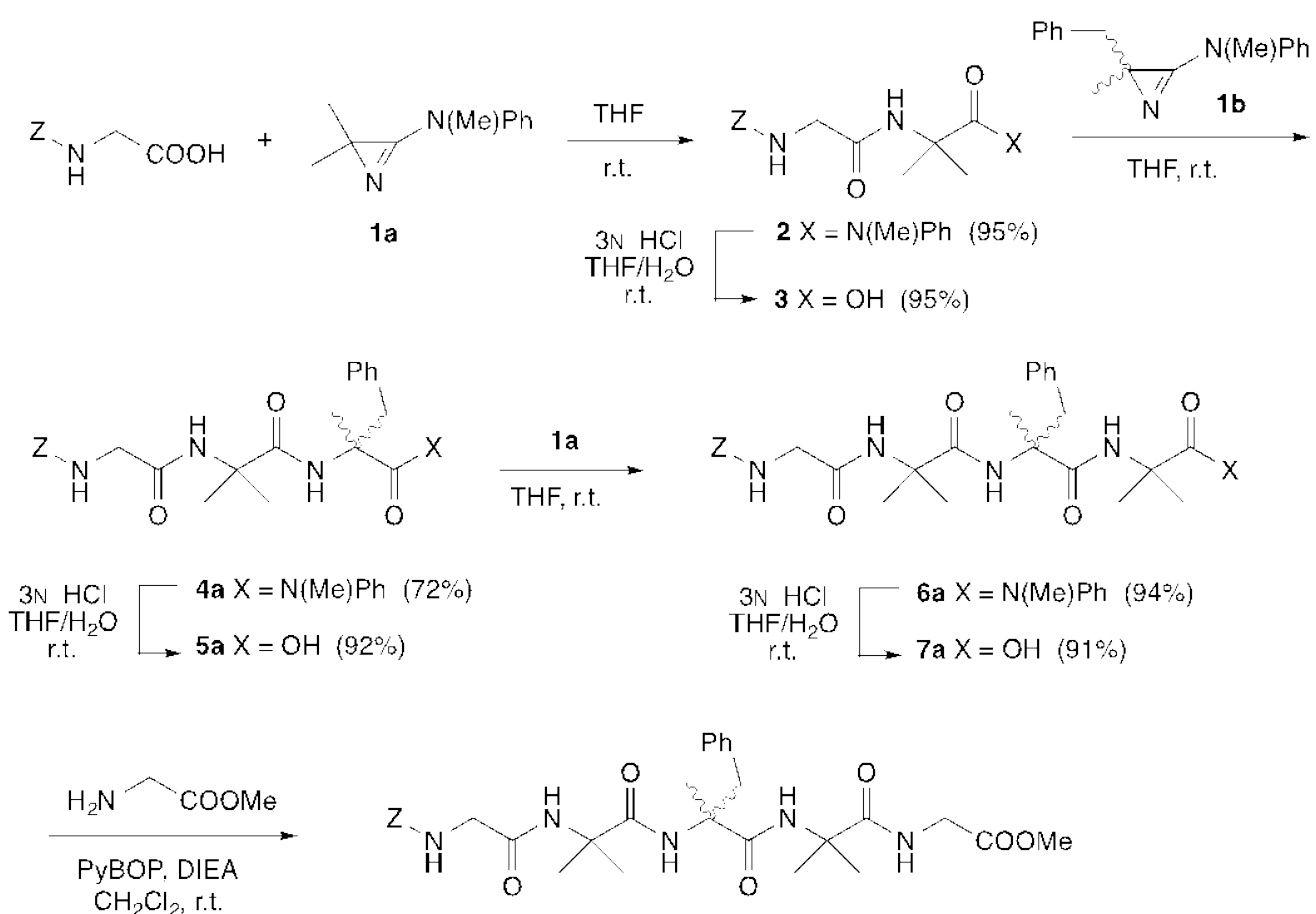

8a $(91 \%)$

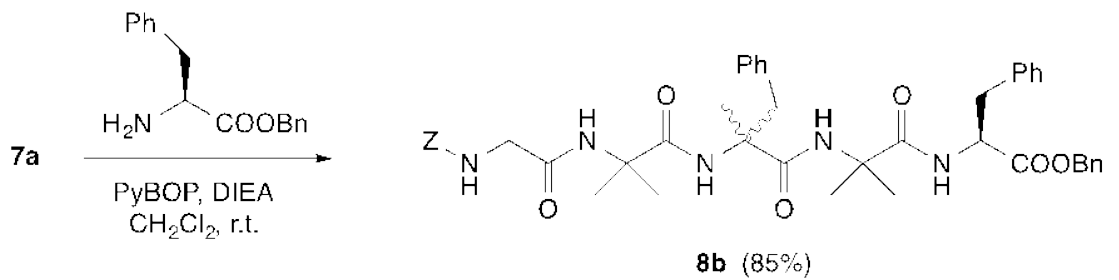

Scheme 2
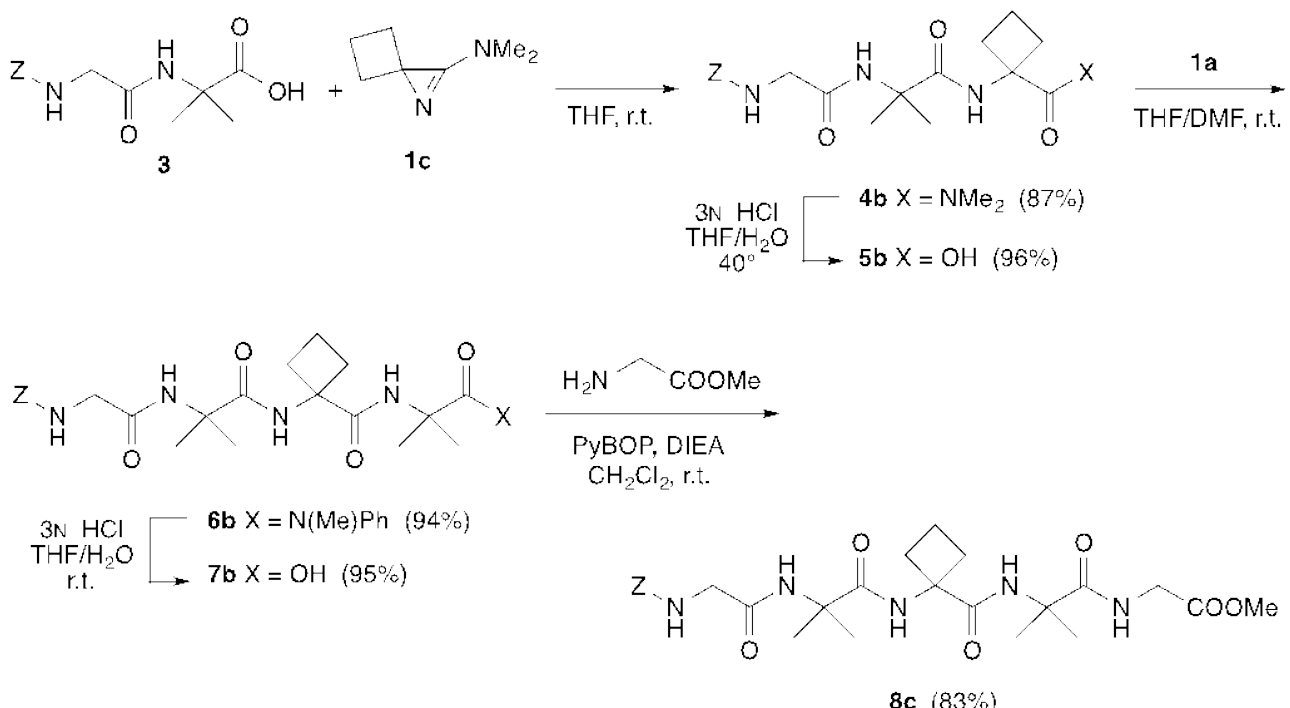


\section{Scheme 3}

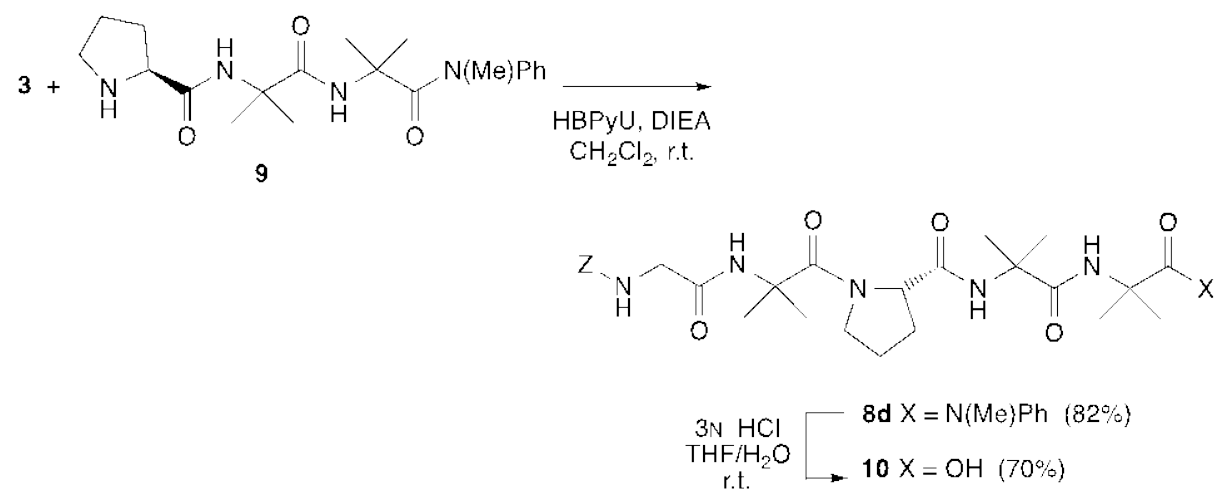

Scheme 4

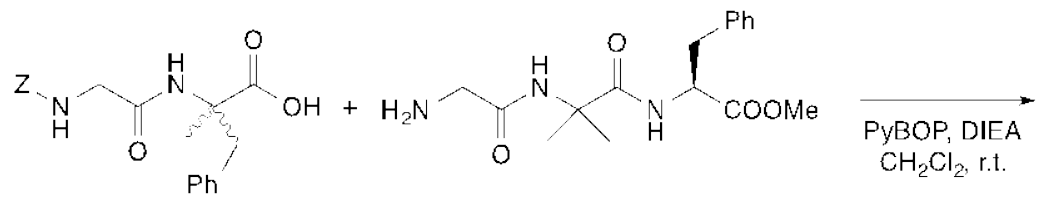

11

12<smiles>[Z]NCC(=O)N[C@@](Cc1ccccc1)(C(=O)NCC(=O)NC(C)(C)C(=O)N[C@@H](Cc1ccccc1)C(C)(C)C)C(C)(C)C</smiles><smiles>[Z]NCC(=O)NC(Cc1ccccc1)C(=O)NCC(=O)NC(C)(C)C(=O)NC(C)(C)C(C)=O</smiles> 
Scheme 5

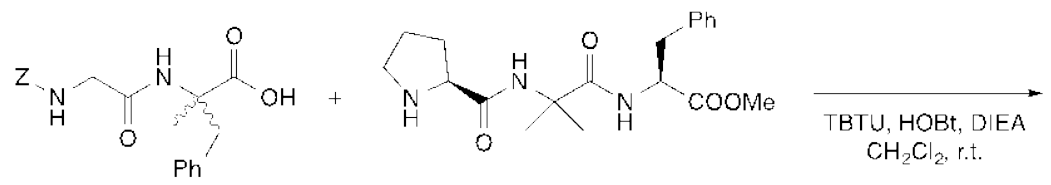

11

13

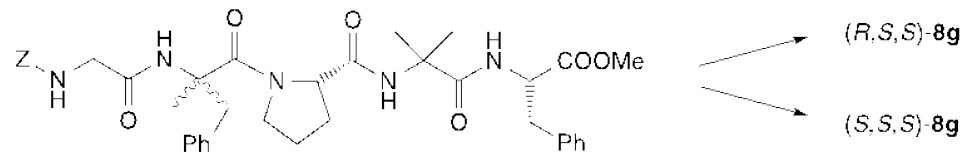

$8 g(74 \%)$

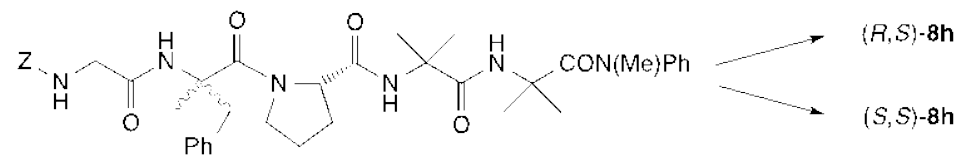

$8 h(72 \%)$

Formula for Table 2

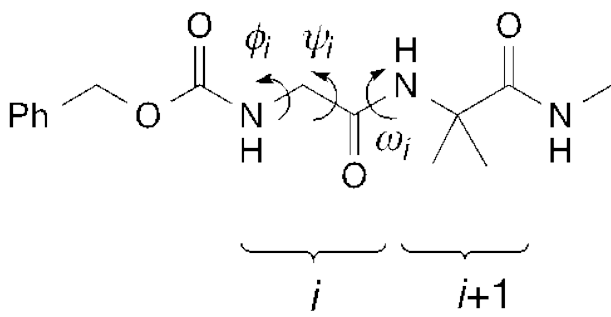

Graphical Abstract

Z. r.t.
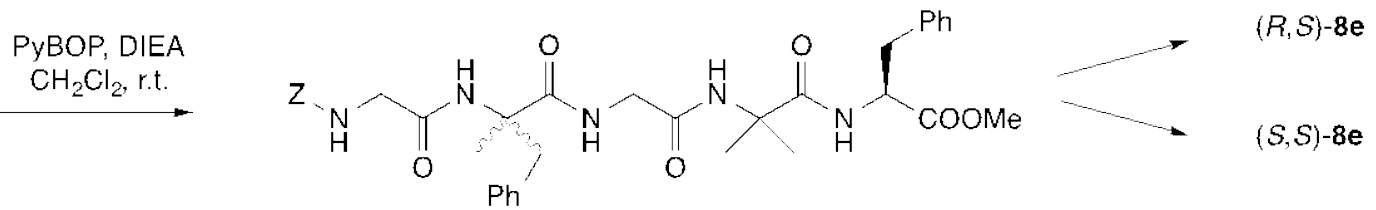
Figure 1

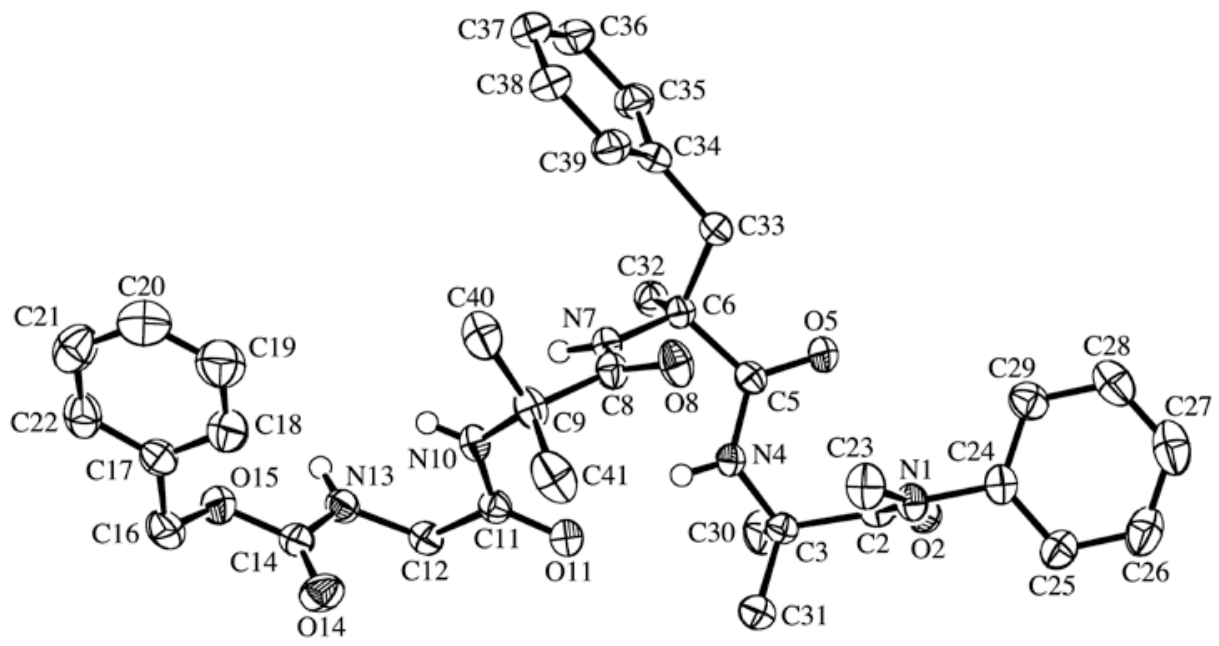

Figure 2

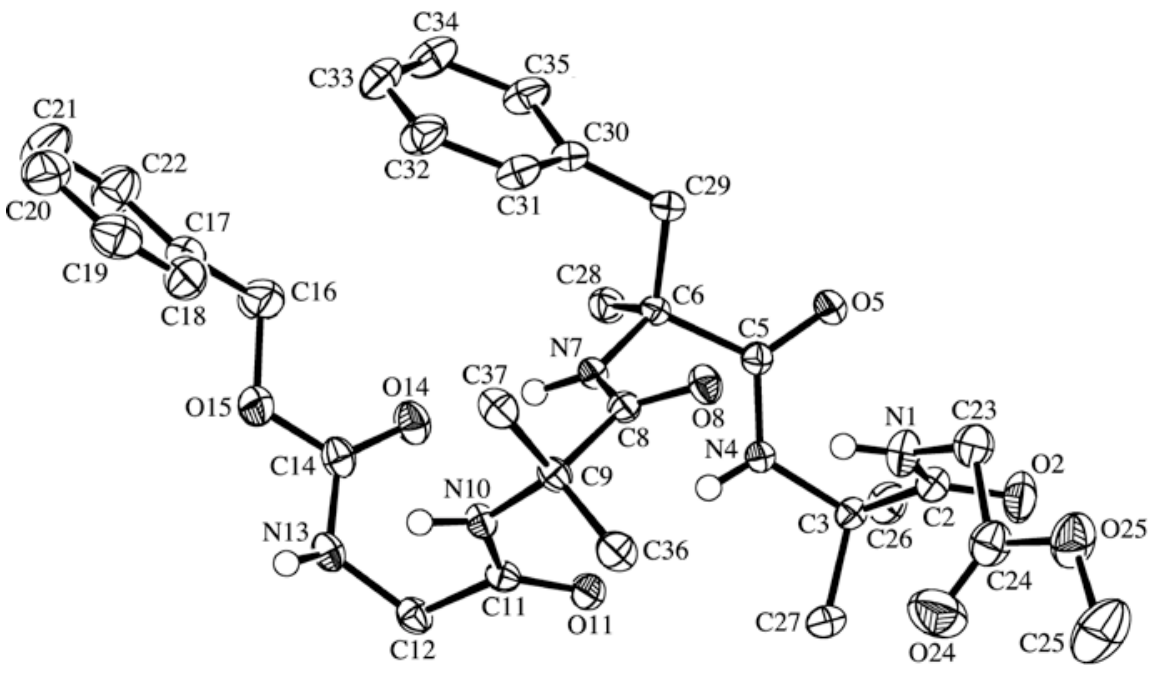


Figure 3

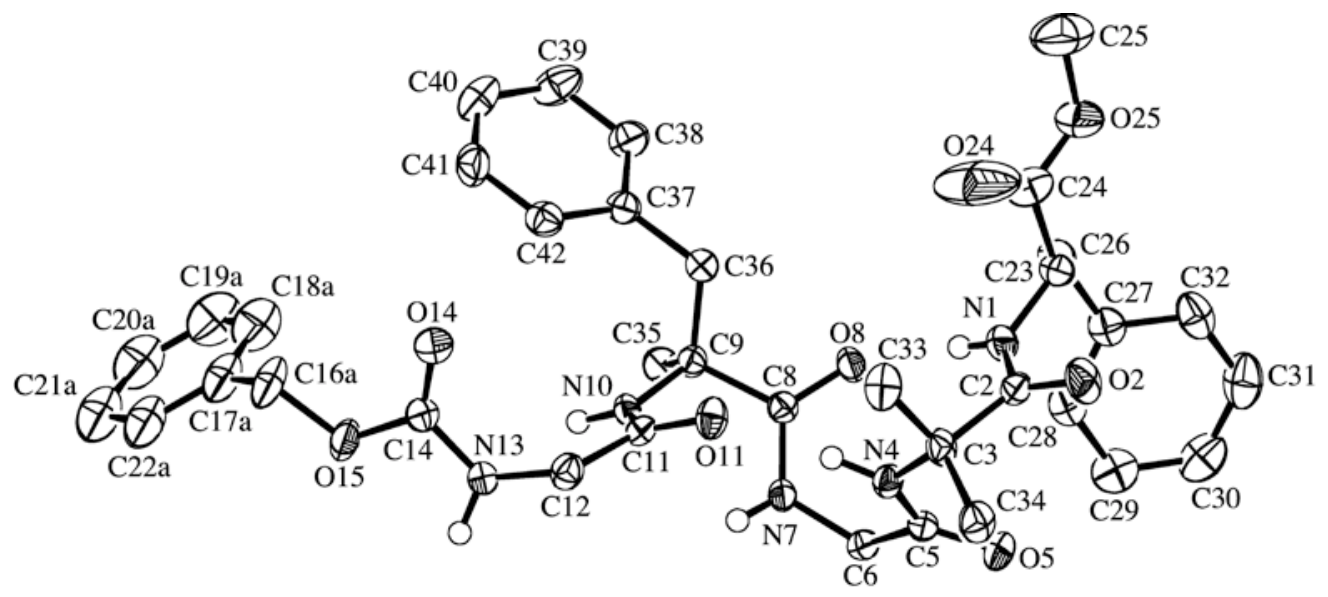

Figure 4

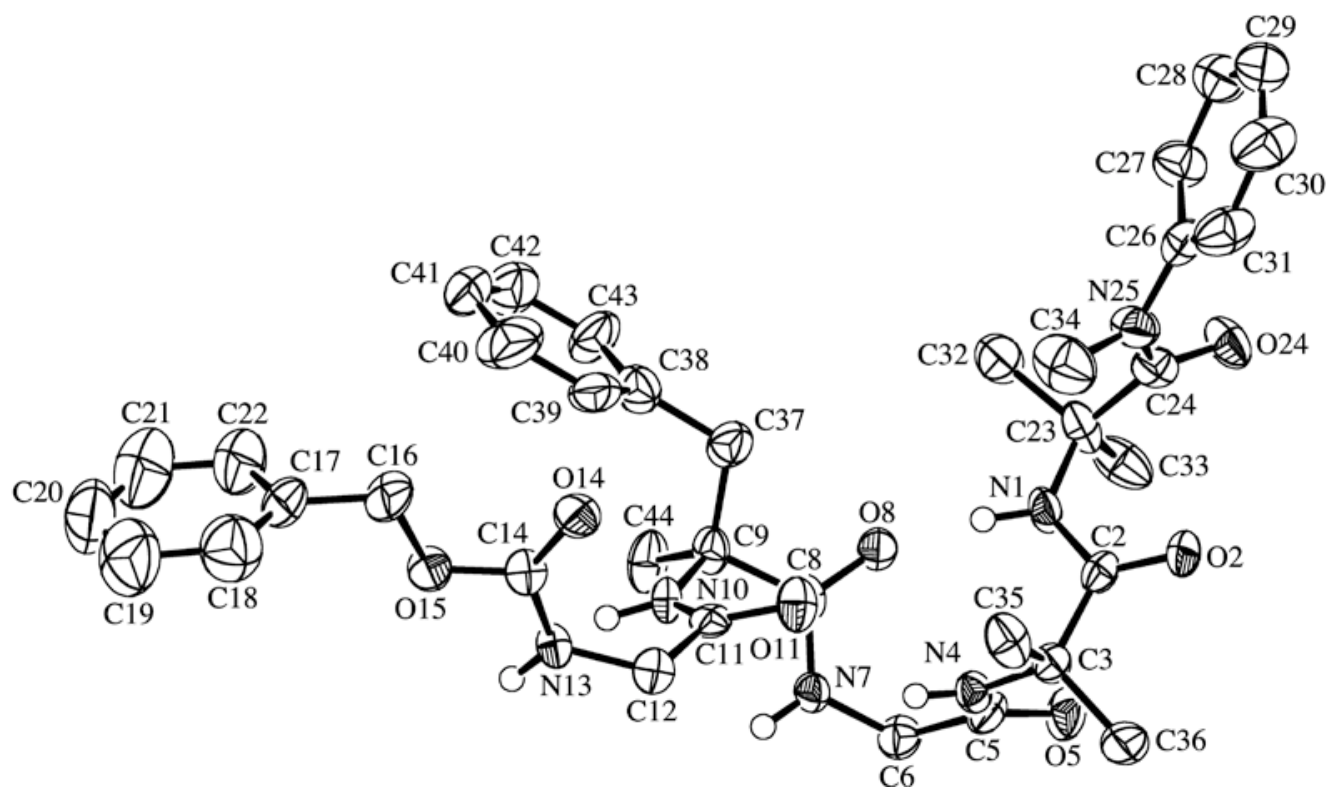


Figure 5

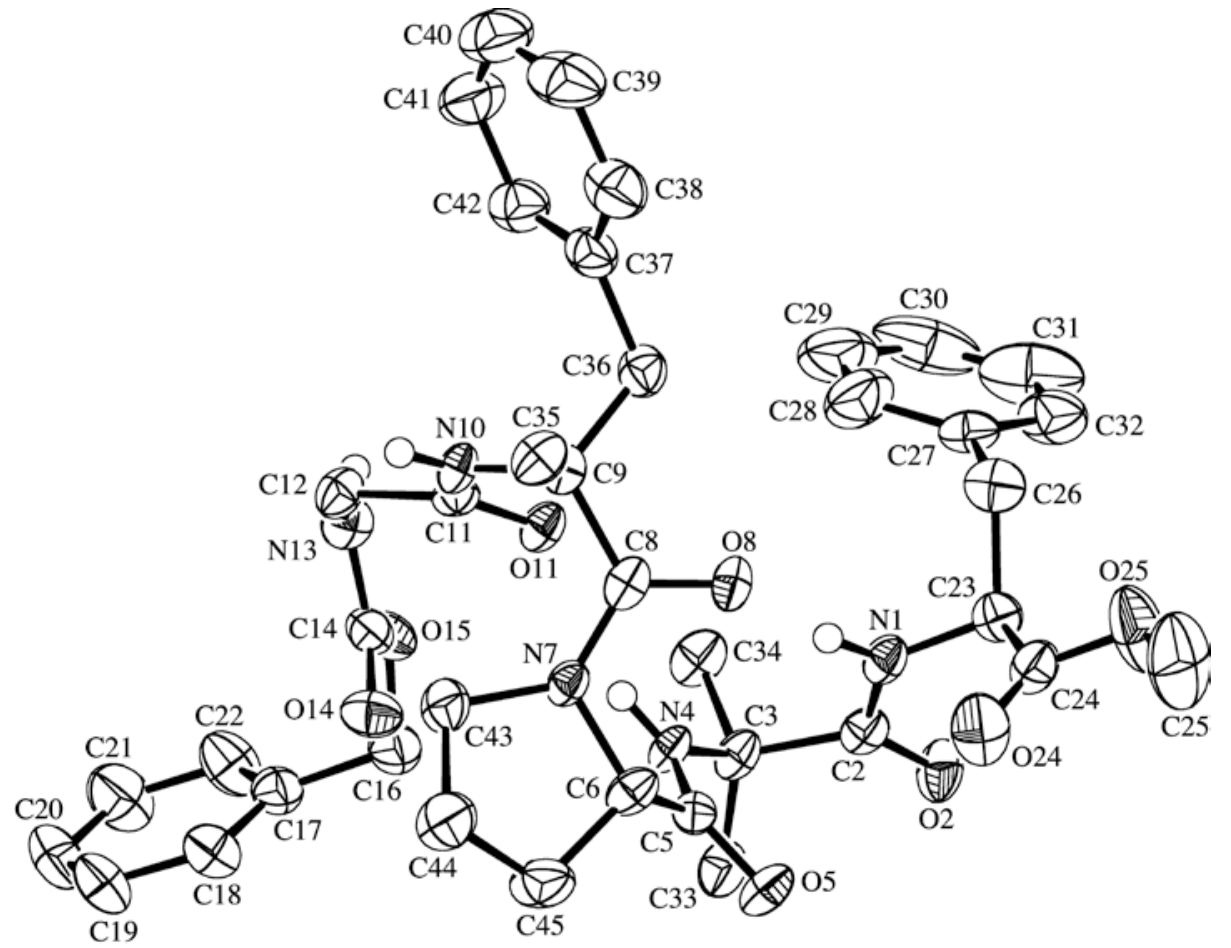

Figure 6

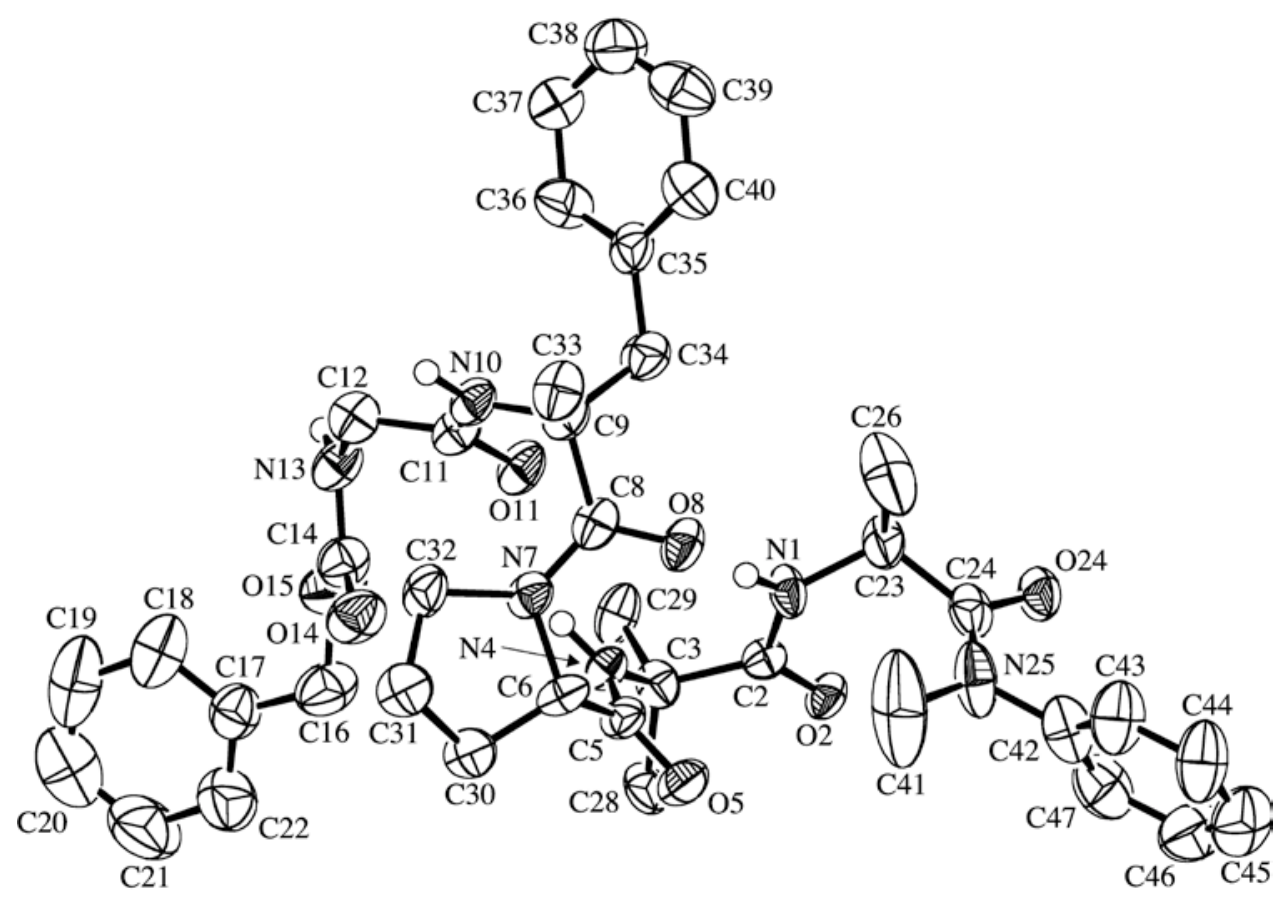


Figure 7

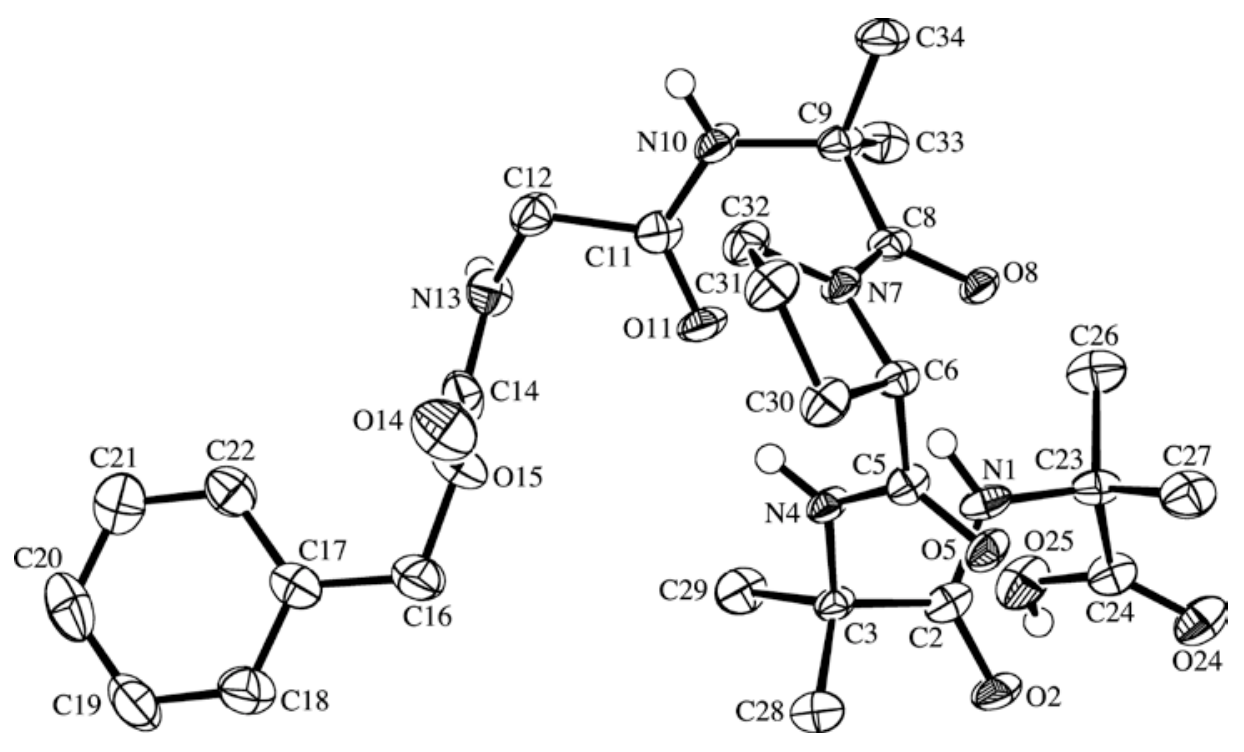

